\title{
Boundary value problems with the transmission property
}

\author{
B.-W. Schulze
}

\begin{abstract}
We give a survey on the calculus of (pseudo-differential) boundary value problems with the transmision property at the boundary, and ellipticity in the Shapiro-Lopatinskij sense. Apart from the original results of the work of Boutet de Monvel we present an approach based on the ideas of the edge calculus. In a final section we introduce symbols with the anti-transmission property.
\end{abstract}

\section{Introduction}

Boundary value problems (BVPs) for elliptic (pseudo-)differential operators have attracted mathematicians and physicists during all periods of the modern analysis. While the definition of ellipticity of an operator on an open (smooth) manifold is very simple, such a notion in connection with a (smooth or non-smooth) boundary is much less evident. During the past few years the interest in BVPs increased again considerably, motivated by new applications and also by unsolved problems in the frame of the structural understanding of ellipticity in new situations. Several classical periods of the development created deep and beautiful ideas, for instance, in connection with function theory, potential theory, with boundary operators satisfying the complementing condition, cf. Agmon, Douglas, Nirenberg [1], or pseudo-differential theories from Vishik and Eskin [29], Eskin [7], Boutet de Monvel [4]. Other branches of the development concern ellipticity with global projection conditions (analogues of Atiyah, Patodi, Singer conditions, cf. [3]), or elliptic theories on manifolds with geometric singularities, cf. the author's papers [25] or [26].

After all that it is not easy to imagine how many basic and interesting problems remained open. A part of the new developments is connected with the analysis on configurations with singularities that includes boundary value problems. In that context it seems to be desirable to see the pseudo-differential machinery of Boutet de Monvel and also of Vishik and Eskin from an alternative viewpoint, using the

2000 Mathematics Subject Classification. 35J40, 58J32, 58J40.

Key words and phrases. pseudo-differential boundary value problems, transmission and antitransmission property, boundary symbolic calculus, Shapiro-Lopatinskij ellipticity, parametrices. 
achievements of the cone and edge pseudo-differential calculus as is pointed out in [16], [20], and in the author's joint paper with Seiler [22], see also the monographs [18], or those jointly with Egorov [6], Kapanadze [12], Harutyunyan [10].

Our exposition just intends to emphasize such an approach, here mainly focused on operators with the transmission property at the boundary from the work of Boutet de Monvel. We also introduce symbols with the anti-transmission property at the boundary. Together with those with the transmission property they span the space of all (classical) symbols that are smooth up to the boundary. A pseudo-differential calculus for such general symbols needs more tools from the edge algebra than developed here.

The present paper is the elaborated version of introductory lectures, given during an International Workshop on Pseudo-Differential Operators, Complex Analysis and Partial Differential Equations at York University on August 4-8, 2008 , in Toronto.

\section{Interior and boundary symbols for differential operators}

Let $X$ be a $C^{\infty}$ manifold with boundary $Y=\partial X$. Moreover, let $2 X$ be the double, defined by gluing together two copies $X_{ \pm}$of $X$ to a $C^{\infty}$ manifold along the common boundary $Y$. Let us fix a Riemannian metric on $2 X$ and consider $Y$ in the induced metric. There is then a tubular neighbourhood of $Y$ in $2 X$ that can be identified with $Y \times[-1,1]$, with a splitting of variables $x=(y, t)$, where $t$ is the variable normal to the boundary and $y \in Y$. We assume that $(y, t)$ belongs to $X=: X_{+}$ for $0 \leq t \leq 1$ and to $X_{-}$for $-1 \leq t \leq 0$.

If $M$ is a $C^{\infty}$ manifold (with or without boundary) by $\operatorname{Diff}^{\mu}(M)$ we denote the set of all differential operators of order $\mu$ on $M$ with smooth coefficients (smooth up to the boundary when $\partial M \neq \emptyset$ ).

Local descriptions near $Y$ will refer to charts

$$
\chi: U \rightarrow \Omega \times \mathbb{R}
$$

for open $U \subseteq 2 X, U \cap Y \neq \emptyset$, and open $\Omega \subseteq \mathbb{R}^{n-1}$, and induced charts

$$
\chi: U \cap Y \rightarrow \Omega
$$

on $Y$ and

$$
\chi_{ \pm}: U_{ \pm}:=U \cap X_{ \pm} \rightarrow \Omega \times \overline{\mathbb{R}}_{ \pm}
$$

on $X_{ \pm}$near the boundary. Concerning the transition maps $\Omega \times \mathbb{R} \rightarrow \widetilde{\Omega} \times \mathbb{R},(y, t) \rightarrow$ $(\tilde{y}, \tilde{t})$, for simplicity we assume that the normal variable remains unchanged near the boundary, i.e., $t=\tilde{t}$ for $|t|$ sufficiently small. The map $y \rightarrow \tilde{y}$ corresponds to a diffeomorphism $\Omega \rightarrow \widetilde{\Omega}$.

Let $A \in \operatorname{Diff}^{\mu}(X), B_{j} \in \operatorname{Diff}^{\mu_{j}}\left(V_{+}\right), V_{+}:=V \cap X, j=1, \ldots, N$, for some $N \in \mathbb{N}$, and set

$$
T u:=\left(\left.B_{j} u\right|_{Y}\right)_{j=1, \ldots N} .
$$


Then the equations

$$
A u=f \operatorname{in} \operatorname{int} X, \quad T u=g \text { on } Y
$$

represent a boundary value problem for $A$. Consider for the moment functions in $C^{\infty}(X)$; then $(2.1)$ can be regarded as a continuous operator

$$
\mathcal{A}=\left(\begin{array}{l}
A \\
T
\end{array}\right): C^{\infty}(X) \rightarrow \underset{C^{\infty}\left(Y, \mathbb{C}^{N}\right)}{C^{\infty}(X)}
$$

If $X$ is compact, we have the standard Sobolev spaces $H^{s}(2 X)$ on $2 X$ and

$$
H^{s}(\operatorname{int} X):=\left.H^{s}(2 X)\right|_{\operatorname{int} X},
$$

$s \in \mathbb{R}$. Then (2.2) extends to continuous operators

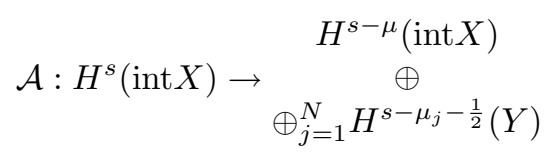

for all $s>\max \left\{\mu_{j}+\frac{1}{2}: j=1, \ldots, N\right\}$.

We will give a survey on elliptic boundary value problems (BVPs), starting from (2.2), and we ask to what extent we may expect a pseudo-differential calculus (an algebra) that contains the operators (2.2) together with the parametrices of elliptic elements. First we have to explain what we understand by ellipticity of a boundaray value problem.

In contrast to the notion of ellipticity of a differential operator (or a, say, classical pseudo-differential operator) $A$ on an open $C^{\infty}$ manifold $M$, in the case of a manifold with boundary we have from the very beginning a variety of choices.

Let $L_{\mathrm{cl}}^{\mu}(M)$ for an open $C^{\infty}$ manifold $M$ denote the space of all classical pseudo-differential operators of order $\mu \in \mathbb{R}$ on $M$. An $A \in L_{\mathrm{cl}}^{\mu}(M)$ is called elliptic, if its homogeneous principal symbol $\sigma_{\psi}(A)(x, \xi)$ of order $\mu$ never vanishes on $T^{*} M \backslash 0$ (the cotangent bundle of $M$ minus the zero section). The union of spaces $L_{\mathrm{cl}}^{\mu}(M)$ over $\mu \in \mathbb{R}$ is closed under the construction of parametrices of elliptic elements, to be more precise, every elliptic $A \in L_{\mathrm{cl}}^{\mu}(M)$ has a (properly supported) parametrix $P \in L_{\mathrm{cl}}^{-\mu}(M)$ such that $1-P A, 1-A P \in L^{-\infty}(M)$ (here and in future by 1 we often denote identity operators). The space $L^{-\infty}(M)$ can be identified with $C^{\infty}(M \times M)$ via a fixed Riemannian metric on $M$.

Let us recall the well-known fact that when $M$ is compact and closed the ellipticity of $A \in L_{\mathrm{cl}}^{\mu}(M)$ is equivalent to the property that

$$
A: H^{s}(M) \rightarrow H^{s-\mu}(M)
$$

is a Fredholm operator for some $s=s_{0} \in \mathbb{R}$. Moreover, from the Fredholm property of (2.4) for $s=s_{0}$ it follows that (2.4) is Fredholm for all $s \in \mathbb{R}$. In addition it is known that $L_{\mathrm{cl}}^{\mu}(M)$ for every $\mu \in \mathbb{R}$ contains so-called order reducing operators, i.e., elliptic operators $R^{\mu}$ that induce isomorphisms,

$$
R^{\mu}: H^{s}(M) \rightarrow H^{s-\mu}(M)
$$


for all $s \in \mathbb{R}$; then $\left(R^{\mu}\right)^{-1} \in L_{\mathrm{cl}}^{-\mu}(M)$ is again order reducing (of opposite order). Below we shall establish more tools on pseudo-differential operators.

Let us now return to BVPs of the form (2.3), where $X$ is a compact manifold with smooth boundary $Y$.

Writing our differential operator $A$ in local coordinates $x \in \Omega \times \overline{\mathbb{R}}_{+}$near the boundary as

$$
A=\sum_{|\alpha| \leq \mu} a_{\alpha}(x) D_{x}^{\alpha},
$$

$a_{\alpha} \in C^{\infty}\left(\Omega \times \overline{\mathbb{R}}_{+}\right)$, we define

$$
\sigma_{\psi}(A)(x, \xi)=\sum_{|\alpha|=\mu} a_{\alpha}(x) \xi^{\alpha},
$$

$(x, \xi) \in T^{*}\left(\Omega \times \overline{\mathbb{R}}_{+}\right) \backslash 0$, and observe the homogeneity

$$
\sigma_{\psi}(A)(x, \lambda \xi)=\lambda^{\mu} \sigma_{\psi}(A)(x, \xi), \lambda \in \mathbb{R}_{+} .
$$

Let $x=(y, t), \xi=(\eta, \tau)$, and set

$$
\sigma_{\partial}(A)(y, \eta)=\sum_{|\alpha|=\mu} a_{\alpha}(y, 0)\left(\eta, D_{t}\right)^{\alpha}
$$

where $\left(\eta, D_{t}\right)^{\alpha}=\eta^{\alpha^{\prime}} D_{t}^{\alpha \prime \prime}$ for $\alpha=\left(\alpha^{\prime}, \alpha^{\prime \prime}\right) \in \mathbb{N}^{n},(y, \eta) \in T^{*} \Omega \backslash 0$, or, equivalently, $\sigma_{\partial}(A)(y, \eta)=\sigma_{\psi}(A)\left(y, 0, \eta, D_{t}\right)$. The expression (2.7) represents a family of continuous operators

$$
\sigma_{\partial}(A): H^{s}\left(\mathbb{R}_{+}\right) \rightarrow H^{s-\mu}\left(\mathbb{R}_{+}\right), s \in \mathbb{R}
$$

called the (homogeneous principal) boundary symbol of $A$.

Let $H^{s}\left(\mathbb{R}_{+}\right)$be endowed with the strongly continuous group $\kappa=\left\{\kappa_{\lambda}\right\}_{\lambda \in \mathbb{R}_{+}}$ of isomorphisms

$$
\kappa_{\lambda}: H^{s}\left(\mathbb{R}_{+}\right) \rightarrow H^{s}\left(\mathbb{R}_{+}\right),\left(\kappa_{\lambda} u\right)(t)=\lambda^{1 / 2} u(\lambda t), \lambda \in \mathbb{R}_{+} .
$$

Then we obtain the following kind of homogeneity of the boundary symbol

$$
\sigma_{\partial}(A)(y, \lambda \eta)=\lambda^{\mu} \kappa_{\lambda} \sigma_{\partial}(A)(y, \eta) \kappa_{\lambda}^{-1}, \lambda \in \mathbb{R}_{+} .
$$

Homogeneity in that sense will also referred to as twisted homogeneity (of order $\mu)$.

It makes sense also to define the (homogeneous principal) boundary symbol of the trace operator $T={ }^{\mathrm{t}}\left(T_{1}, \ldots, T_{N}\right)$, by

$$
\sigma_{\partial}\left(T_{j}\right)(y, \eta) u:=\left.\sigma_{\psi}\left(B_{j}\right)\left(y, 0, \eta, D_{t}\right) u\right|_{t=0},
$$

$u \in H^{s}\left(\mathbb{R}_{+}\right), s>\max \left\{\mu_{j}+\frac{1}{2}: j=1, \ldots, N\right\}$ where $\sigma_{\psi}\left(B_{j}\right)(x, \xi)$ is the homogeneous principal symbol of the operator $B_{j}$, and (2.10) is interpreted as a family of operators

$$
\sigma_{\partial}\left(T_{j}\right)(y, \eta): H^{s}\left(\mathbb{R}_{+}\right) \rightarrow \mathbb{C},
$$

$(y, \eta) \in T^{*} \Omega \backslash 0$. The boundary symbol (2.10) is homogeneous in the sense

$$
\sigma_{\partial}\left(T_{j}\right)(y, \lambda \eta)=\lambda^{\mu_{j}+\frac{1}{2}} \sigma_{\partial}\left(T_{j}\right)(y, \eta) \kappa_{\lambda}^{-1}, \lambda \in \mathbb{R}_{+} .
$$


It is often convenient to compose (2.2) from the left by an operator

$$
\operatorname{diag}\left(1, R_{1}, \ldots, R_{N}\right)
$$

where $R_{j} \in L_{\mathrm{cl}}^{\mu-\left(\mu_{j}+\frac{1}{2}\right)}(Y)$ is an order reducing operator on the boundary in the above-mentioned sense and to pass to a modified operator

$$
\left(\begin{array}{c}
A \\
{ }_{(}\left(R_{1} T_{1}, \ldots, R_{N} T_{N}\right)
\end{array}\right): H^{s}(\operatorname{int} X) \rightarrow \underset{H^{s-\mu}\left(Y, \mathbb{C}^{N}\right)}{\oplus}
$$

related to the former one by a trivial pseudo-differential reduction of orders on the boundary. This is formally a little easier (later on we admit such trace operators anyway). Instead of (2.11) we then obtain

$$
\sigma_{\partial}\left(R_{j} T_{j}\right)(y, \lambda \eta)=\lambda^{\mu} \sigma_{\partial}\left(R_{j} T_{j}\right)(y, \eta) \kappa_{\lambda}^{-1}, \lambda \in \mathbb{R}_{+},
$$

where

$$
\sigma_{\partial}\left(R_{j} T_{j}\right)(y, \eta)=\sigma_{\psi}\left(R_{j}\right)(y, \eta) \sigma_{\partial}\left(T_{j}\right)(y, \eta)
$$

with $\sigma_{\partial}\left(R_{j}\right)(y, \eta)$ being the homogeneous principal symbol of $R_{j}$ of order $\mu-\left(\mu_{j}+\right.$ $\left.\frac{1}{2}\right)$ as a classical pseudo-differential operator on the boundary.

Let us now explain the role of the trace operators in connection with the ellipticity of a boundary value problem. We call the pair

$$
\sigma(\mathcal{A})=\left(\sigma_{\psi}(\mathcal{A}), \sigma_{\partial}(\mathcal{A})\right)
$$

the principal symbol of $\mathcal{A}$, consisting of the (principal) interior symbol $\sigma_{\psi}(\mathcal{A}):=$ $\sigma_{\psi}(A)$ and the (principal) boundary symbol $\sigma_{\partial}(\mathcal{A}):={ }^{\mathrm{t}}\left(\sigma_{\partial}(A), \sigma_{\partial}\left(T_{1}\right), \ldots, \sigma_{\partial}\left(T_{N}\right)\right)$ of $\mathcal{A}$,

$$
\sigma_{\partial}(\mathcal{A}): H^{s}\left(\mathbb{R}_{+}\right) \rightarrow \underset{\mathbb{C}^{N}}{\oplus^{s-\mu}\left(\mathbb{R}_{+}\right)}
$$

Ellipticity of $\mathcal{A}$ requires the bijectivity of both components on $T^{*} X \backslash 0$ and $T^{*} Y \backslash 0$, respectively, the latter as an operator function for $s-\mu>-\frac{1}{2}$. Since the operators $\sigma_{\partial}\left(T_{j}\right)(y, \eta)$ are of finite rank, $\sigma_{\partial}(A)(y, \eta)$ has to be a family of Fredholm operators. The following lemma shows that this is an automatic consequence of the ellipticity of $A$ with respect to $\sigma_{\psi}$.

Lemma 2.1. Let $A$ be an elliptic differential operator; then

$$
\sigma_{\partial}(A)(y, \eta): H^{s}\left(\mathbb{R}_{+}\right) \rightarrow H^{s-\mu}\left(\mathbb{R}_{+}\right)
$$

is a surjective family of Fredholm operators for every real $s>\mu-\frac{1}{2}$, and the kernel $\operatorname{ker} \sigma_{\partial}(A)(y, \eta)$ is a finite-dimensional subspace of $\mathcal{S}\left(\overline{\mathbb{R}}_{+}\right)=\left.\mathcal{S}(\mathbb{R})\right|_{\overline{\mathbb{R}}_{+}}$which is independent of $s$. Moreover, $\operatorname{dim} \operatorname{ker} \sigma_{\partial}(A)(y, \eta)=\operatorname{dim} \operatorname{ker} \sigma_{\partial}(A)(y, \eta /|\eta|)$ for all $(y, \eta) \in T^{*} Y \backslash 0$. 
Proof. Set for the moment $a(\tau):=\sigma_{\psi}(A)(y, 0, \eta, \tau)$ with frozen variables $(y, \eta)$, $\eta \neq 0$. Then $\sigma_{\partial}(\mathcal{A})=a\left(D_{t}\right)$ can be written as op ${ }^{+}(a):=\mathrm{r}^{+} \mathrm{op}_{t}(a) \mathrm{e}^{+}$for the operator of extension $\mathrm{e}^{+}$of functions by 0 to $t<0$ and $\mathrm{r}^{+}$the restriction to $t>0$, and $\operatorname{op}_{t}(\cdot)$ is the pseudo-differential operator on $\mathbb{R}$ with the symbol $a(\tau)$, i.e., $\mathrm{op}_{t}(a) u(t)=\iint e^{i\left(t-t^{\prime}\right) \tau} a(\tau) u\left(t^{\prime}\right) d t^{\prime} d \tau, d \tau=(2 \pi)^{-1} d \tau$. Then op ${ }^{+}\left(a^{-1}\right)$ is a right inverse of op op $^{+}(a)$, since op op $^{+}(a) \mathrm{op}^{+}\left(a^{-1}\right)=\mathrm{op}^{+}\left(a a^{-1}\right)+\mathrm{r}^{+} \mathrm{op}(a) \mathrm{e}^{-} \mathrm{op}^{+}\left(a^{-1}\right)=1$, because of $\mathrm{r}^{+} \mathrm{op}(a) \mathrm{e}^{-}=0$. This shows the surjectivity of op $\mathrm{pp}^{+}(a)$. The fact that solutions $u$ of the homogeneous equation $a\left(D_{t}\right) u=0$ form a finite-dimensional subspace of $\mathcal{S}\left(\overline{\mathbb{R}}_{+}\right)$is standard. However, we will show those things below once again independently, cf. Theorem 3.29 below. The last assertion follows from the homogeneity (2.9).

Example. Let $A=\Delta$ be the Laplacian, $\Delta=\sum_{j=1}^{n} \frac{\partial^{2}}{\partial x_{j}^{2}}$ in local variables. Then $\sigma_{\partial}(\Delta)=-|\xi|^{2}$, and

$$
\sigma_{\partial}(\Delta)(\eta)=-|\eta|^{2}+D_{t}^{2}: H^{s}\left(\mathbb{R}_{+}\right) \rightarrow H^{s-2}\left(\mathbb{R}_{+}\right) .
$$

We have

$$
\operatorname{ker} \sigma_{\partial}(\Delta)(\eta)=\left\{c e^{-|\eta| t}: c \in \mathbb{C}\right\},
$$

i.e., $\operatorname{dim} \operatorname{ker} \sigma_{\partial}(\Delta)(\eta)=1$ for all $\eta \neq 0$ and all $s>3 / 2$.

Remark 2.2. The operators $T_{k}: H^{s}(X) \rightarrow H^{s-k-1 / 2}(Y)$, locally near $Y$ defined by

$$
T_{k} u:=\left.D_{t}^{k} u\right|_{t=0}, k \in \mathbb{N},
$$

have the boundary symbols

$$
\sigma_{\partial}\left(T_{k}\right) u=\left.D_{t}^{k} u\right|_{t=0}, \quad \sigma_{\partial}\left(T_{k}\right)(\eta): H^{s}\left(\mathbb{R}_{+}\right) \rightarrow \mathbb{C}
$$

and are (although they are independent of $\eta$ ) of homogeneity $k+\frac{1}{2}$, i.e.,

$$
\sigma_{\partial}\left(T_{k}\right)(\lambda \eta) u=\lambda^{k+\frac{1}{2}} \sigma_{\partial}\left(T_{k}\right)(\eta) \kappa_{\lambda}^{-1} u, \lambda \in \mathbb{R}_{+} .
$$

Moreover, as we see from Lemma 2.1 together with Lemma 2.3 below, the column matrix

$$
\left(\begin{array}{c}
\sigma_{\partial}(\Delta)(\eta) \\
\sigma_{\partial}\left(T_{k}\right)(\eta)
\end{array}\right): H^{s}\left(\mathbb{R}_{+}\right) \rightarrow{\stackrel{\oplus}{H^{s-2}\left(\mathbb{R}_{+}\right)}}^{\oplus}
$$

is an isomorphism for every $\eta \neq 0, s>\max \left\{\frac{3}{2}, k+\frac{1}{2}\right\}$; this is true of every $k \in \mathbb{N}$. Observe that $T_{0}$ represents Dirichlet and $T_{1}$ Neumann conditions.

In other words, the boundary symbol $\sigma_{\partial}\left(T_{k}\right)$ fills up the Fredholm operators $\sigma_{\partial}(\Delta)(\eta): H^{s}\left(\mathbb{R}_{+}\right) \rightarrow H^{s-2}\left(\mathbb{R}_{+}\right), s>3 / 2$, to a family of isomorphisms (2.14). In this way we have examples of so-called elliptic BVPs, namely,

$$
\mathcal{A}_{k}:=\left(\begin{array}{c}
\Delta \\
T_{k}
\end{array}\right): H^{s}(X) \rightarrow \begin{gathered}
H^{s-2}(X) \\
H^{s-k-\frac{1}{2}}(Y)
\end{gathered}
$$


for every $k \in \mathbb{N}$. In connection with such constructions it is useful to recall the following simple algebraic result.

Lemma 2.3. Let $H, \widetilde{H}, \widetilde{L}$ be Hilbert spaces and $a: H \rightarrow \widetilde{H}, b: H \rightarrow \underset{\widetilde{L}}{\widetilde{H}}$ linear $\widetilde{H}$

continuous operators. Then the column matrix operator $\boldsymbol{a}:=\left(\begin{array}{l}a \\ b\end{array}\right): H \rightarrow \underset{\widetilde{L}}{\oplus}$ is an isomorphism if and only if $a: H \rightarrow \widetilde{H}$ is surjective, and $b: H \rightarrow \widetilde{L}$ restricts to an isomorphism $\left.b\right|_{\text {ker } a}: \operatorname{ker} a \rightarrow \widetilde{L}$.

Proof. Let $a: H \rightarrow \widetilde{H}$ be surjective, and $b_{0}:=\left.b\right|_{\text {ker } a}: \operatorname{ker} a \rightarrow \widetilde{L}$ an isomorphism. Then $\boldsymbol{a}$ is obviously surjective. Moreover, $\boldsymbol{a} u=0$ implies $u \in \operatorname{ker} a$ and $b_{0} u=0$; then, since $b_{0}$ is an isomorphism it follows that $u=0$. Thus $\boldsymbol{a}$ is injective and hence an isomorphism. Conversely, assume that $\boldsymbol{a}$ is an isomorphism. The surjectivity of $\boldsymbol{a}$ implies that $a: H \rightarrow \widetilde{H}, b: H \rightarrow \widetilde{L}$ are both surjective. In particular, if $H_{1}$ denotes the orthogonal complement of ker $a_{1}$ in $H$ we obtain an isomorphism $a_{1}:=\left.a\right|_{H_{1}}: H_{1} \rightarrow \widetilde{H}$, and $\boldsymbol{a}$ can be written as a block matrix

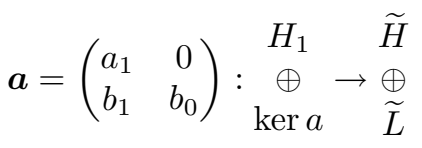

for $b_{1}:=\left.b\right|_{H_{1}}$. It remains to show that $b_{0}: \operatorname{ker} a \rightarrow \widetilde{L}$ is an isomorphism. The operator $\left(\begin{array}{cc}a_{1}^{-1} & 0 \\ -b_{1} a_{1}^{-1} & 1\end{array}\right): \underset{\widetilde{L}}{\underset{\widetilde{H}}{\oplus}} \rightarrow \underset{\widetilde{L}}{\oplus}$ is an isomorphism, and we have

$$
\left(\begin{array}{cc}
a_{1}^{-1} & 0 \\
-b_{1} a_{1}^{-1} & 1
\end{array}\right)\left(\begin{array}{cc}
a_{1} & 0 \\
b_{1} & b_{0}
\end{array}\right)=\left(\begin{array}{cc}
1 & 0 \\
0 & b_{0}
\end{array}\right): \underset{\operatorname{ker} a}{\oplus} \underset{\widetilde{L}}{H_{1}} \underset{\stackrel{\oplus}{\oplus}}{H_{1}}
$$

Therefore, since both factors on the left hand side are isomorphisms, it follows that also $b_{0}: \operatorname{ker} a \rightarrow \operatorname{ker} a$ is an isomorphism.

This shows us the meaning of the above-mentioned $N$, the number of trace operators which turns the boundary symbol

$$
\sigma_{\partial}(\mathcal{A})(y, \eta): H^{s}\left(\mathbb{R}_{+}\right) \rightarrow \stackrel{H^{s-\mu}\left(\mathbb{R}_{+}\right)}{\bigoplus^{N}}
$$

to a family of isomorphisms. According to Lemmas 2.1 and 2.3 for an elliptic differential operator $A$ we have $N=\operatorname{dim} \operatorname{ker} \sigma_{\partial}(A)(y, \eta)$; this number is required to be independent of $y$ and $\eta \neq 0$. As is well-known, if $A$ is of order $2 m$ and admits boundary operators $\left\{B_{1}, \ldots, B_{m}\right\}$ satisfying the so-called complementing condition with respect to $A$, then (for $m=N$ ) that property holds (cf. Agmon, Douglis, Nirenberg [1], Lions, Magenes [13]). 
Definition 2.4. The block matrix operator $\mathcal{A}={ }^{\mathrm{t}}\left(\begin{array}{ll}A & T\end{array}\right)$ is said to be a (ShapiroLopatinskij) elliptic boundary value problem for the elliptic differential operator $A$ if the boundary symbol (2.16) is a family of isomorphisms for any sufficiently large $s$, for all $(y, \eta) \in T^{*} Y \backslash 0$. We also talk about Shapiro-Lopatinskij trace (or boundary) conditions for the operator $A$.

Remark 2.5. Observe that not every elliptic differential operator $A$ admits ShapiroLopatinskij elliptic trace conditions. The simplest example is the Cauchy-Riemann operator $\bar{\partial}$ in the complex plane. More general examples are Dirac operators in even dimensions, and other important geometric operators. We will return later on to this discussion in the context of the Atiyah-Bott obstruction for the existence of Shapiro-Lopatinskij elliptic conditions.

If we ask an algebra of BVPs a first essential formal problem is that column matrices cannot be composed with each other in a reasonable manner. However, we extend the notion "algebra" and talk about block matrix operators where the algebraic operations are carried out only under natural conditions, namely, addition when the matrices have the same number of rows and columns and multiplication when the number of rows and columns in the middle fit together. For instance, if we consider the Dirichlet problem $\mathcal{A}_{0}$ for the Laplacian, cf. the formula (2.15) for $k=0$, we have invertibility of

$$
\mathcal{A}_{0}: C^{\infty}(X) \rightarrow \underset{C^{\infty}(Y)}{\stackrel{C^{\infty}(X)}{\oplus}}
$$

Denoting by $\mathcal{P}:=\left(\begin{array}{ll}P_{0} & K_{0}\end{array}\right)$ the inverse of $\mathcal{A}_{0}$ (which belongs to the pseudodifferential operator calculus to be discussed here) then we have two kinds of compositions, namely, for $A=\Delta$

$$
\mathcal{A}_{0} \mathcal{P}_{0}=\left(\begin{array}{c}
A \\
T_{0}
\end{array}\right)\left(\begin{array}{ll}
P_{0} & K_{0}
\end{array}\right)=\left(\begin{array}{cc}
A P_{0} & A K_{0} \\
T_{0} P_{0} & T_{0} K_{0}
\end{array}\right)=\left(\begin{array}{ll}
1 & 0 \\
0 & 1
\end{array}\right),
$$

and

$$
\left(\begin{array}{ll}
P_{0} & K_{0}
\end{array}\right)\left(\begin{array}{c}
A \\
T_{0}
\end{array}\right)=P_{0} A+K_{0} T_{0}=1 .
$$

It also makes sense to consider

$$
\mathcal{A}_{k} \mathcal{P}_{0}=\left(\begin{array}{cc}
1 & 0 \\
T_{k} P_{0} & T_{k} K_{0}
\end{array}\right)
$$

for every $k \in \mathbb{N}$. The lower right corner of the latter matrix has the meaning of the reduction of the boundary condition $T_{k}$ to the boundary (by means of the Dirichlet problem). It turns out that $T_{k} K_{0}$ is a classical elliptic pseudo-differential operator of order $k$ on the boundary. Its symbol will be computed in the following section, cf. the formula (3.28). 


\section{Inverses of boundary symbols}

Let us first recall that the construction of a parametrix of an elliptic operator $A \in$ $L_{\mathrm{cl}}^{\mu}(M)$ on an open $C^{\infty}$ manifold $M$ can be started by inverting the homogeneous principal symbol and forming a $B \in L_{\mathrm{cl}}^{-\mu}(M)$ such that $\sigma_{\psi}(B)=\sigma_{\psi}^{-1}(A)$ ( $B$ is obtained via an operator convention). In a second step we form

$$
1-B A=C \in L_{\mathrm{cl}}^{-1}(M)
$$

(everything in the frame of properly supported pseudo-differential operators), then we pass to a $D \in L_{\mathrm{cl}}^{-1}(M)$ such that $(1+D)(1-C)=1 \bmod L^{-\infty}(M)$. Such a $D$ can be found as an asymptotic sum $\sum_{j=1}^{\infty} C^{j}$, and $P=(1+D) B$ is then a left parametrix of $A$. (For future references we call the latter procedure a formal Neumann series argument.) In an analogous manner we find a right parametrix, and then a simple algebraic consideration shows that $P$ is a two-sided parametrix.

These arguments are based on the following properties of (classical) pseudodifferential operators:

1. every pseudo-differential operator has a properly supported representative modulo a smoothing operator;

2. any sequence of operators of order $\mu-j, j \in \mathbb{N}$, has an asymptotic sum, uniquely determined modulo a smoothing operator;

3. there is a symbolic map that assigns the unique principal symbol of an operator; the algebraic operations between operators are compatible with those for associated principal symbols (in particular, the principal symbol of a composition is equal to the composition (product) of the principal symbols);

4. every smooth homogeneous function of order $\mu$ on $T^{*} M \backslash 0$ is the principal symbol of an associated pseudo-differential operator of order $\mu$ (i.e., there is an operator convention that is right inverse of the principal symbolic map of 3.);

5. an operator of order $\mu$ with vanishing principal symbol is of order $\mu-1$.

It turns out that boundary value problems as in Section 2 can be completed to a graded algebra of $2 \times 2$ block matrix operators with a two-component principal symbolic hierarchy $\sigma=\left(\sigma_{\psi}, \sigma_{\partial}\right)$, where analogues of the properties 1 . - 5. hold. Such an algebra has been introduced by Boutet de Monvel [4], and we discuss here (among other things) some elements of that calculus.

The first essential point is to analyse the nature of inverses of bijective boundary symbols. Since such inverses are computed $(y, \eta)$-wise for $(y, \eta) \in T^{*} Y \backslash 0$ we first freeze those variables and look at operators on $\mathbb{R}_{+}$. Let us consider a classical symbol $a(\tau) \in S_{\mathrm{cl}}^{\mu}(\mathbb{R}), \mu \in \mathbb{R}$. Examples of such symbols are

$$
l_{ \pm}^{\mu}(\tau):=(1 \pm i \tau)^{\mu} .
$$

Let us set

$$
\mathrm{op}^{+}(a) u(t)=\mathrm{r}^{+} \mathrm{op}(a)\left(\mathrm{e}^{+} u\right)(t),
$$


for every $u \in H^{s}\left(\mathbb{R}_{+}\right) \quad\left(=\left.H^{s}(\mathbb{R})\right|_{\mathbb{R}_{+}}\right), s>-\frac{1}{2}$, where $\mathrm{e}^{+} u \in \mathcal{S}^{\prime}(\mathbb{R})$ is the distribution obtained by extending $u$ by zero to $\mathbb{R}_{-}$, i.e.,

$$
\mathrm{e}^{+} u(t)=u(t) \text { for } t>0, \mathrm{e}^{+} u(t)=0 \text { for } t<0 .
$$

Moreover $\mathrm{r}^{+}$is the operator of restriction from $\mathbb{R}$ to $\mathbb{R}_{+}$, and

$$
\mathrm{op}(a) v(t)=\iint e^{i\left(t-t^{\prime}\right) \tau} a(\tau) u\left(t^{\prime}\right) d t^{\prime} d \tau,
$$

$d \tau=(2 \pi)^{-1} d \tau$. In an analogous manner we define the extension $\mathrm{e}^{-}$by zero from $\mathbb{R}_{-}$to $\mathbb{R}$ and the restriction $\mathrm{r}^{-}$from $\mathbb{R}$ to $\mathbb{R}_{-}$. The operator (3.2) defines a linear map

$$
\mathrm{op}^{+}(a): H^{s}\left(\mathbb{R}_{+}\right) \rightarrow \mathcal{S}^{\prime}\left(\mathbb{R}_{+}\right)
$$

for every $s>-\frac{1}{2}, \mathcal{S}^{\prime}\left(\mathbb{R}_{+}\right):=\left.\mathcal{S}^{\prime}(\mathbb{R})\right|_{\mathbb{R}_{+}}$. As is well-known (cf. [7]) in some cases $\mathrm{op}^{+}(\cdot)$ induces a continuous operator

$$
\mathrm{op}^{+}(a): H^{s}\left(\mathbb{R}_{+}\right) \rightarrow H^{s-\mu}\left(\mathbb{R}_{+}\right)
$$

for every $s>-1 / 2$, namely, when $a(\tau)$ is a so-called minus-symbol.

Let $\mathcal{A}(U), U \subseteq \mathbb{C}$ open, denote the space of all holomorphic functions in $U$, and set $\mathbb{C}_{ \pm}:=\{z=\tau+i \beta: \beta \gtrless 0\}$. Then $a(\tau) \in S_{\mathrm{cl}}^{\mu}(\mathbb{R})$ is said to be a minus-symbol if $a(\tau)$ has an extension to a function in $\mathcal{A}\left(\mathbb{C}_{+}\right) \cap C^{\infty}\left(\overline{\mathbb{C}}_{+}\right)$such that

$$
|a(z)| \leq c\left(1+|z|^{2}\right)^{\mu / 2}
$$

for all $z \in \overline{\mathbb{C}}_{+}$, for some constant $c>0$. By a plus-symbol of order $\mu$ we understand an element $a(\tau) \in S_{\mathrm{cl}}^{\mu}(\mathbb{R})$ that extends to a function in $\mathcal{A}\left(\mathbb{C}_{-}\right) \cap C^{\infty}\left(\overline{\mathbb{C}}_{-}\right)$such that the estimates (3.4) hold for all $z \in \overline{\mathbb{C}}_{-}$. For $s \in \mathbb{R}$ we have a relation similar to (3.3) when we replace $\mathrm{e}^{+}$by a continuous extension operator $\mathrm{e}_{s}^{+}: H^{s}\left(\mathbb{R}_{+}\right) \rightarrow H^{s}(\mathbb{R})$ with $\left.\mathrm{e}_{s}^{+} u\right|_{\mathbb{R}_{+}}=u$; then in the minus-case the latter map is independent of the choice of $\mathrm{e}_{s}^{+}$.

If $a(\tau)$ is a plus-symbol of order $\mu$ and $H_{0}^{s}\left(\overline{\mathbb{R}}_{+}\right):=\left\{u \in H^{s}(\mathbb{R}): u=0\right.$ on $\left.\mathbb{R}_{-}\right\}$, then

$$
\mathrm{op}^{+}(a): H_{0}^{s}\left(\mathbb{R}_{+}\right) \rightarrow H_{0}^{s-\mu}\left(\mathbb{R}_{+}\right)
$$

is continuous for every $s \in \mathbb{R}$. Concerning a proof of the continuity of (3.3) and (3.5), see [7, Lemma 4.6 and Theorem 4.4], (cf. also [10, Section 4.1.2]). Moreover, for an arbitrary $p(\tau) \in S_{\mathrm{cl}}^{\nu}(\mathbb{R}), \nu \in \mathbb{R}$, we have

$$
\mathrm{op}^{+}(a p)=\mathrm{op}^{+}(a) \mathrm{op}^{+}(p)
$$

when $a(\tau) \in S_{\mathrm{cl}}^{\mu}(\mathbb{R})$ is a minus-symbol (since $\left.\mathrm{r}^{+} \mathrm{op}(a) \mathrm{e}^{-}=0\right)$ and

$$
\mathrm{op}^{+}(p a)=\mathrm{op}^{+}(p) \mathrm{op}^{+}(a)
$$

when $a(\tau) \in S_{\mathrm{cl}}^{\mu}(\mathbb{R})$ is a plus-symbol (since $\mathrm{r}^{-} \mathrm{op}(a) \mathrm{e}^{+}=0$ ).

Example. A polynomial in $\tau$ is both a minus- and a plus-symbol. 
Remark 3.1. The function $l_{-}^{\mu}(\tau)=(1-i \tau)^{\mu}$ is a minus-symbol of order $\mu \in \mathbb{R}$, and

$$
\mathrm{op}^{+}\left(l_{-}^{\mu}\right): H^{s}\left(\mathbb{R}_{+}\right) \rightarrow H^{s-\mu}\left(\mathbb{R}_{+}\right)
$$

is an isomorphism for every $s \in \mathbb{R}, s>\max \left\{-\frac{1}{2}, \mu-\frac{1}{2}\right\}$, where $\left(\mathrm{op}^{+}\left(l_{-}^{\mu}\right)\right)^{-1}=$ $\mathrm{op}^{+}\left(l_{-}^{-\mu}\right)$. Moreover, $l_{+}^{\mu}(\tau)=(1+i \tau)^{\mu}$ is a plus-symbol of order $\mu \in \mathbb{R}$, and

$$
\mathrm{op}^{+}\left(l_{+}^{\mu}\right): H_{0}^{s}\left(\mathbb{R}_{+}\right) \rightarrow H_{0}^{s-\mu}\left(\mathbb{R}_{+}\right)
$$

is an isomorphism for every $s \in \mathbb{R}$ where $\left(\mathrm{op}^{+}\left(l_{+}^{\mu}\right)\right)^{-1}=\mathrm{op}^{+}\left(l_{+}^{-\mu}\right)$.

A classical symbol $a(\tau) \in S_{\mathrm{cl}}^{\mu}(\mathbb{R})$ has an asymptotic expansion

$$
a(\tau) \sim \sum_{j=0}^{\infty} a_{j}^{ \pm}(i \tau)^{\mu-j} \text { for } \tau \rightarrow \pm \infty
$$

for unique coefficients $a_{j}^{ \pm} \in \mathbb{C}$ (the imaginary unit $i=\sqrt{-1}$ is taken for convenience; powers are defined as $(i \tau)^{\nu}=e^{\nu \log (i \tau)}$ with the principal branch of the logarithm).

If $\chi(\tau) \in C^{\infty}(\mathbb{R})$ is an excision function in $\tau$ (i.e., $\chi(\tau)=0$ for $|\tau|<c_{0}$, $\chi(\tau)=1$ for $|\tau|>c_{1}$, for some $\left.0<c_{0}<c_{1}\right)$ then we have

$$
a(\tau) \sim \sum_{j=0}^{\infty} \chi(\tau) a_{(\mu-j)}(\tau)
$$

for

$$
a_{(\mu-j)}(\tau)=\left\{a_{j}^{+} \theta^{+}(\tau)+a_{j}^{-} \theta^{-}(\tau)\right\}(i \tau)^{\mu-j},
$$

with $\theta^{+}$being the characteristic function of the \pm half-axis in $\tau$, where (3.9) has the meaning of an asymptotic expansion of symbols, $\chi(\tau) a_{(\mu-j)}(\tau) \in S_{\mathrm{cl}}^{\mu-j}(\mathbb{R})$.

Definition 3.2. A symbol $a(\tau) \in S_{\mathrm{cl}}^{\mu}(\mathbb{R})$ for $\mu \in \mathbb{Z}$ has the transmission property if

$$
a_{j}^{+}=a_{j}^{-} \text {for all } j \in \mathbb{N} \text {. }
$$

Let $S_{\mathrm{tr}}^{\mu}(\mathbb{R})$ denote the space of all symbols in $S_{\mathrm{cl}}^{\mu}(\mathbb{R})$ with the transmission property.

Remark 3.3. A symbol $a(\tau) \in S_{\mathrm{cl}}^{\mu}(\mathbb{R})$ has the transmission property exactly when

$$
a_{(\mu-j)}(\tau)=(-1)^{\mu-j} a_{(\mu-j)}(-\tau)
$$

for all $\tau \in \mathbb{R} \backslash\{0\}$ and all $j \in \mathbb{N}$.

In fact, the transmission property means that $a_{(\mu-j)}(\tau)=c_{j}(i \tau)^{\mu-j}$ for $c_{j}:=$ $a_{j}^{+}=a_{j}^{-}$for all $j \in \mathbb{N}$, and this shows the relation (3.12). Conversely from (3.12) we deduce

$$
\begin{aligned}
\left\{a_{j}^{+} \theta^{+}(\tau)+a_{j}^{-} \theta^{-}(\tau)\right\}(i \tau)^{\mu-j} & =(-1)^{\mu-j}\left\{a_{j}^{+} \theta^{+}(-\tau)+a_{j}^{-} \theta^{-}(-\tau)\right\}(-i \tau)^{\mu-j} \\
& =\left\{a_{j}^{+} \theta^{+}(-\tau)+a_{j}^{-} \theta^{-}(-\tau)\right\}(i \tau)^{\mu-j}
\end{aligned}
$$

for all $\tau \neq 0$, which implies $a_{j}^{+}\left(\theta^{+}(\tau)-\theta^{+}(-\tau)\right)=a_{j}^{-}\left(\theta^{-}(-\tau)-\theta^{-}(\tau)\right)$. For $\tau>0$ we have $\theta^{+}(-\tau)=\theta^{-}(\tau)=0$ and $\theta^{+}(\tau)=\theta^{-}(-\tau)=1$ which yields $a_{j}^{+}=a_{j}^{-}$. 
Remark 3.4. The space $S_{\mathrm{cl}}^{\mu}(\mathbb{R})$ is a nuclear Fréchet space in a natural way, and $S_{\mathrm{tr}}^{\mu}(\mathbb{R})$ is a closed subspace in the induced topology.

Example. $\quad$ 1. Every polynomial in $\tau$ has the transmission property;

2 . the $\tau$-wise product of two symbols with the transmission property has again the transmission property;

3. If $a \in S_{\mathrm{tr}}^{\mu}(\mathbb{R})$ and $a_{0}^{+}=a_{0}^{-} \neq 0$ then it follows that $\chi(\tau) a^{-1}(\tau) \in S_{\mathrm{tr}}^{-\mu}(\mathbb{R})$ for a suitable excision function $\chi(\tau)$. If in addition $a(\tau) \neq 0$ for all $\tau \in \mathbb{R}$ then $a^{-1}(\tau) \in S_{\mathrm{tr}}^{-\mu}(\mathbb{R})$.

In particular, the symbols (3.1) for $\mu \in \mathbb{Z}$ have the transmission property.

Remark 3.5. The multiplication of symbols by $l_{+}^{-\mu}(\tau)\left(\right.$ or $\left.l_{-}^{-\mu}(\tau)\right)$ induces an isomorphism

$$
S_{\mathrm{tr}}^{\mu}(\mathbb{R}) \rightarrow S_{\mathrm{tr}}^{0}(\mathbb{R})
$$

Remark 3.6. Let $a(\tau) \in S_{\mathrm{cl}}^{0}(\mathbb{R})$, and form the bounded set $L(a):=\{a(\tau) \in \mathbb{C}$ : $\tau \in \mathbb{R}\}$ which is a smooth curve (with admitted self-intersections) and end points $a_{0}^{ \pm}=a( \pm \infty)$. Then we have $a(\tau) \in S_{\mathrm{tr}}^{0}(\mathbb{R})$ if and only if $L(a)$ is a closed curve which is smooth including $a_{0}^{+}=a_{0}^{-}$.

Remark 3.7. Every symbol $a(\tau) \in S_{\mathrm{tr}}^{\mu}(\mathbb{R})$ can be written in the form

$$
a(\tau)=p(\tau)+b(\tau)
$$

where $p(\tau)$ is a polynomial in $\tau$ of order $\mu$ (only relevant for $\mu \geq 0$ ) and $b(\tau) \in$ $S_{\operatorname{tr}}^{-1}(\mathbb{R})$.

In fact, this is an evident consequence of Definition 3.2.

Proposition 3.8. Let $a(\tau) \in S_{\mathrm{tr}}^{\mu}(\mathbb{R})$; then for every $N \in \mathbb{N}$ there is a minus-symbol $m_{N}(\tau) \in S_{\mathrm{cl}}^{\mu}(\mathbb{R})$ and a plus-symbol $p_{N}(\tau) \in S_{\mathrm{cl}}^{\mu}(\mathbb{R})$ such that $a(\tau)-m_{N}(\tau) \in$ $S_{\mathrm{cl}}^{-(N+1)}(\mathbb{R})$ and $a(\tau)-p_{N}(\tau) \in S_{\mathrm{cl}}^{-(N+1)}(\mathbb{R})$.

Proof. Since a polynomial in $\tau$ is a plus- and a minus-symbol it suffices to assume $\mu=-1$. By definition there are constants $a_{j}$ such that for any fixed excision function $\chi(\tau)$

$$
a(\tau)=\chi(\tau) \sum_{j=1}^{N} a_{j}(i \tau)^{-j}+r_{N}(\tau)
$$

where $r_{N}(\tau) \in S_{\mathrm{cl}}^{-(N+1)}(\mathbb{R})$. The relation $\frac{1}{i \tau}=-\frac{1}{1-i \tau}+\frac{1}{i \tau} \frac{1}{1-i \tau}$ can be iterated, and we obtain $\frac{1}{i \tau}=-\frac{1}{1-i \tau}+\left\{-\frac{1}{1-i \tau}+\frac{1}{i \tau} \frac{1}{1-i \tau}\right\} \frac{1}{1-i \tau}=-\frac{1}{1-i \tau}-\frac{1}{(1-i \tau)^{2}}+\frac{1}{i \tau} \frac{1}{(1-i \tau)^{2}}=$ $\ldots=-\sum_{k=1}^{N} \frac{1}{(1-i \tau)^{k}}+\frac{1}{i \tau} \frac{1}{(1-i \tau)^{N}}$. This yields $(i \tau)^{-j}=\left(-\sum_{k=1}^{N}(1-i \tau)^{-k}\right)^{j}+$ $r_{j, N}(\tau)$ for every $j \in \mathbb{N} \backslash\{0\}$ where $\chi(\tau) r_{j, N}(\tau) \in S_{\mathrm{cl}}^{-(N+1)}(\mathbb{R})$ for every excision function $\chi(\tau)$. Thus, setting $m_{j, N}(\tau):=a_{j}\left(-\sum_{k=1}^{N}(1-i \tau)^{-k}\right)^{j}$ we obtain

$$
\chi(\tau) a_{j}(i \tau)^{-j}=m_{j, N}(\tau)+\chi(\tau) a_{j} r_{j, N}(\tau)
$$


modulo a symbol in $S^{-\infty}(\mathbb{R})$, where $m_{j, N}(\tau)$ is a minus-symbol, cf. Remark 3.1. Then from (3.13) we obtain the first assertion, for $m_{N}(\tau)=\sum_{j=1}^{N} m_{j, N}(\tau)$. Moreover, writing $\frac{1}{i \tau}=\frac{1}{1+i \tau}+\frac{1}{i \tau} \frac{1}{1+i \tau}=\sum_{k=1}^{N} \frac{1}{(1+i \tau)^{k}}+\frac{1}{i \tau} \frac{1}{(1+i \tau)^{N}}$ we obtain a plussymbol $p_{N}(\tau):=\sum_{j=1}^{N} p_{j, N}(\tau), p_{j, N}(\tau):=a_{j}\left(\sum_{k=1}^{N}(1+i \tau)^{-k}\right)^{j}$ with the desired property.

Corollary 3.9. Let $a(\tau) \in S_{\mathrm{tr}}^{\mu}(\mathbb{R})$; then $\mathrm{op}^{+}(a)$ induces a continuous operator

$$
\mathrm{op}^{+}(a): H^{s}\left(\mathbb{R}_{+}\right) \rightarrow H^{s-\mu}\left(\mathbb{R}_{+}\right)
$$

for every real $s>-\frac{1}{2}$.

Proof. Let us write $\mathrm{op}(a)=\mathrm{op}\left(m_{N}\right)+\mathrm{op}\left(c_{N}\right)$ where, according to Proposition $3.8, m_{N}$ is a minus-symbol of order $\mu$, and $c_{N} \in S_{\mathrm{cl}}^{-(N+1)}(\mathbb{R})$. Then we have

$$
\mathrm{op}^{+}(a)=\mathrm{op}^{+}\left(m_{N}\right)+\mathrm{op}^{+}\left(c_{N}\right) \text {. }
$$

We observed before that $\operatorname{op}^{+}\left(m_{N}\right)$ has the desired mapping property. Let us now assume $s \in\left(-\frac{1}{2}, 0\right]$. We employ the known fact that for those $s$ we have $\mathrm{e}^{+} H^{s}\left(\mathbb{R}_{+}\right)=H_{0}^{s}\left(\mathbb{R}_{+}\right)$. As noted before we have an isomorphism

$$
\operatorname{op}\left(l_{+}^{s}\right): H_{0}^{s}\left(\mathbb{R}_{+}\right) \rightarrow L^{2}\left(\mathbb{R}_{+}\right)=H_{0}^{0}\left(\mathbb{R}_{+}\right)
$$

with the inverse op ${ }^{+}\left(l_{+}^{-s}\right)$. Moreover, using the relation (3.7) we have

$$
\mathrm{op}^{+}\left(c_{N}\right)=\mathrm{op}^{+}\left(c_{N} l_{+}^{-s}\right) \mathrm{op}^{+}\left(l_{+}^{s}\right)
$$

where $\left(c_{N} l_{+}^{-s}\right)(\tau) \in S_{\mathrm{cl}}^{-(N+1)-s}(\mathbb{R})$. Thus it remains to verify that op ${ }^{+}\left(c_{N} l_{+}^{-s}\right)$ : $L^{2}\left(\mathbb{R}_{+}\right) \rightarrow H^{s-\mu}\left(\mathbb{R}_{+}\right)$is continuous. However, when $N$ is large enough, we have the continuity

$$
\mathrm{op}^{+}\left(c_{N} l_{+}^{-s}\right): L^{2}\left(\mathbb{R}_{+}\right) \rightarrow H^{N+1+s}\left(\mathbb{R}_{+}\right) .
$$

Thus for $N$ so large that $N+1 \geq-\mu$ we obviously obtain the desired continuity. Finally for $s \geq 0$ it suffices to employ the continuous embedding $\mathrm{e}^{+} H^{s}\left(\mathbb{R}_{+}\right) \hookrightarrow$ $L^{2}(\mathbb{R})$, i.e., we can argue similarly as before and obtain the continuity op ${ }^{+}\left(c_{N}\right)$ : $L^{2}\left(\mathbb{R}_{+}\right) \rightarrow H^{s-\mu}\left(\mathbb{R}_{+}\right)$for $N+1>s-\mu$.

Proposition 3.10. Every symbol $a(\tau) \in S_{\mathrm{tr}}^{-1}(\mathbb{R})$ can be written in the form

$$
a(\tau)=a_{+}(\tau)+a_{-}(\tau)
$$

for uniquely determined

$$
a_{+}(\tau) \in F_{t \rightarrow \tau}\left(\mathrm{e}^{+} \mathcal{S}\left(\overline{\mathbb{R}}_{+}\right)\right), \quad a_{-}(\tau) \in F_{t \rightarrow \tau}\left(\mathrm{e}^{-\mathcal{S}}\left(\overline{\mathbb{R}}_{-}\right)\right)
$$

which are plus/minus symbols in $S_{\mathrm{tr}}^{-1}(\mathbb{R})$.

Concerning a proof of Proposition 3.10, see [15, Section 2.1.1.1].

Corollary 3.11. Let $a(\tau) \in S_{\mathrm{tr}}^{\mu}(\mathbb{R})$; then $\mathrm{op}^{+}(a)$ induces a continuous operator

$$
\mathrm{op}^{+}(a): \mathcal{S}\left(\overline{\mathbb{R}}_{+}\right) \rightarrow \mathcal{S}\left(\overline{\mathbb{R}}_{+}\right) \text {. }
$$


Proof. For $\mu \in \mathbb{N}$ the symbol $a(\tau)$ is equal to a polynomial in $\tau$ of order $\mu$, modulo a symbol in $S_{\mathrm{tr}}^{-1}(\mathbb{R})$. Thus without loss of generality we assume $\mu=-1$. The Fourier transform $F=F_{t \rightarrow \tau}$ induces a continuous operator

$$
F: \mathrm{e}^{+} \mathcal{S}\left(\overline{\mathbb{R}}_{+}\right) \rightarrow \mathcal{S}_{\text {tr }}^{-1}(\mathbb{R}) .
$$

Moreover, the multiplication between symbols with the transmission property is bilinear continuous. In particular, the composition of (3.17) with the multiplication by the symbol (3.16) gives us a continuous operator

$$
a(\tau) F: \mathrm{e}^{+} \mathcal{S}\left(\overline{\mathbb{R}}_{+}\right) \rightarrow S_{\mathrm{tr}}^{-1}(\mathbb{R}) .
$$

Finally $F^{-1}: S_{\mathrm{tr}}^{-1}(\mathbb{R}) \rightarrow \mathrm{e}^{+} \mathcal{S}\left(\overline{\mathbb{R}}_{+}\right)+\mathrm{e}^{-} \mathcal{S}\left(\overline{\mathbb{R}}_{-}\right)$is a topological isomorphism (the sum on the right hand side is direct) and

$$
\mathrm{r}^{+}: \mathrm{e}^{+} \mathcal{S}\left(\overline{\mathbb{R}}_{+}\right)+\mathrm{e}^{-} \mathcal{S}\left(\overline{\mathbb{R}}_{-}\right) \rightarrow \mathcal{S}\left(\overline{\mathbb{R}}_{+}\right)
$$

is obviously continuous. Thus $\operatorname{op}^{+}(a)=\mathrm{r}^{+} F^{-1} a(\tau) F \mathrm{e}^{+}$is a composition of continuous operators.

Proposition 3.12. Let $a(\tau) \in S_{\mathrm{tr}}^{0}(\mathbb{R})$; then the adjoint of

$$
\text { op }^{+}(a): L^{2}\left(\mathbb{R}_{+}\right) \rightarrow L^{2}\left(\mathbb{R}_{+}\right)
$$

with respect to the $L^{2}\left(\mathbb{R}_{+}\right)$-scalar product has the form op ${ }^{+}(\bar{a})$ for the complex conjugate $\bar{a}(\tau) \in S_{\mathrm{tr}}^{0}(\mathbb{R})$.

Proof. The computation is completely elementary.

Proposition 3.13. Let $a(\tau) \in S_{\mathrm{cl}}^{0}(\mathbb{R})$ be a symbol with the transmission property, let $\varepsilon: \mathbb{R}_{ \pm} \rightarrow \mathbb{R}_{\mp}$ be defined by $\varepsilon(t)=-t$, and $\varepsilon^{*}: L^{2}\left(\mathbb{R}_{ \pm}\right) \rightarrow L^{2}\left(\mathbb{R}_{\mp}\right)$ the corresponding function pull back. Then

$$
\mathrm{r}^{+} \mathrm{op}(a) \mathrm{e}^{-} \varepsilon^{*}, \varepsilon^{*} \mathrm{r}^{-} \mathrm{op}(a) \mathrm{e}^{+}: L^{2}\left(\mathbb{R}_{+}\right) \rightarrow L^{2}\left(\mathbb{R}_{+}\right)
$$

induce continuous operators $L^{2}\left(\mathbb{R}_{+}\right) \rightarrow \mathcal{S}\left(\overline{\mathbb{R}}_{+}\right)$.

Proof. If $a(\tau)$ is a constant both operators are zero. Therefore, it suffices to assume $a(\tau) \in S_{\mathrm{cl}}^{-1}(\mathbb{R})$. By virtue of the identity

$$
\mathrm{r}^{+} \mathrm{op}(a)=\mathrm{op}^{+}\left(\left(l_{-}^{N}\right)\left(l_{-}^{-N}\right)\right) \mathrm{r}^{+} \mathrm{op}(a)=\mathrm{op}^{+}\left(l_{-}^{N}\right) \mathrm{r}^{+} \mathrm{op}\left(l_{-}^{-N} a\right)
$$

for any $N \in \mathbb{Z}$ (cf. the relation (3.6) taking into account that $l_{-}^{ \pm N}(\tau)$ are minussymbols) we may even consider the symbol $l_{-}^{-N}(\tau) a(\tau) \in S_{\mathrm{cl}}^{-(N+1)}(\mathbb{R})$ rather than $a(\tau)$, for any $N>1$, since op ${ }^{+}\left(l_{-}^{N}\right): \mathcal{S}\left(\overline{\mathbb{R}}_{+}\right) \rightarrow \mathcal{S}\left(\overline{\mathbb{R}}_{+}\right)$is continuous, cf. Corollary 3.11. In other words, let $a(\tau) \in S_{\mathrm{cl}}^{-2}(\mathbb{R})$; then

$$
\begin{aligned}
\mathrm{r}^{+} \mathrm{op}(a) \mathrm{e}^{-} \varepsilon^{*} v(t) & =\mathrm{r}^{+} \int_{\mathbb{R}} \int_{0}^{\infty} e^{i\left(t+t^{\prime}\right) \tau} a(\tau) v\left(-t^{\prime}\right) d t^{\prime} d \tau \\
& =\mathrm{r}^{+} \int_{0}^{\infty}\left\{\int e^{i\left(t+t^{\prime}\right) \tau} a(\tau) d \tau\right\} v\left(-t^{\prime}\right) d t^{\prime} .
\end{aligned}
$$


By virtue of Proposition 3.10 we have

$$
\int e^{i r \tau} a(\tau) d \tau \in \mathrm{e}^{+} \mathcal{S}\left(\overline{\mathbb{R}}_{+}\right)+\mathrm{e}^{-\mathcal{S}}\left(\overline{\mathbb{R}}_{-}\right)
$$

with $r \in \mathbb{R}$ being the variable on the right hand side. Since $r$ has the meaning of $t+t^{\prime}$ for $t>0, t^{\prime}>0$, we obtain

$$
\mathrm{r}^{+} \mathrm{op}(a) \mathrm{e}^{-} \varepsilon^{*} v(t)=\mathrm{r}^{+} \int_{0}^{\infty} f\left(t+t^{\prime}\right) v\left(-t^{\prime}\right) d t^{\prime}
$$

for some $f(r) \in \mathrm{e}^{+} \mathcal{S}\left(\overline{\mathbb{R}}_{+}\right)$. It remains to observe that the right hand side of (3.19) represents a continuous operator $L^{2}\left(\mathbb{R}_{+}\right) \rightarrow \mathcal{S}\left(\overline{\mathbb{R}}_{+}\right)$. The second operator in (3.18) can be treated in an analogous manner.

Corollary 3.14. Let $g$ denote one of the operators in (3.18), and let $g^{*}$ be its adjoint in $L^{2}\left(\mathbb{R}_{+}\right)$. Then $g$ and $g^{*}$ induce continuous operators

$$
g, g^{*}: L^{2}\left(\mathbb{R}_{+}\right) \rightarrow \mathcal{S}\left(\overline{\mathbb{R}}_{+}\right) .
$$

Proof. The assertion for $g$ is contained in Proposition 3.13. Moreover, because of $\left(\mathrm{r}^{+} \mathrm{op}(a) \mathrm{e}^{-} \varepsilon^{*}\right)^{*}=\varepsilon^{*} \mathrm{r}^{-} \mathrm{op}(\bar{a}) \mathrm{e}^{+}$by Proposition 3.13 we also obtain the result for $g^{*}$.

Remark 3.15. It can be proved that an operator $g \in \mathcal{L}\left(L^{2}\left(\mathbb{R}_{+}\right)\right)$that defines continuous operators $(3.20)$ can be represented in the form

$$
g u(t)=\int_{0}^{\infty} c\left(t, t^{\prime}\right) u\left(t^{\prime}\right) d t^{\prime}
$$

for some $c\left(t, t^{\prime}\right) \in \mathcal{S}\left(\overline{\mathbb{R}}_{+} \times \overline{\mathbb{R}}_{+}\right)\left(=\left.\mathcal{S}(\mathbb{R} \times \mathbb{R})\right|_{\overline{\mathbb{R}}_{+} \times \overline{\mathbb{R}}_{+}}\right)$, see [10, Theorem 2.4.87].

Definition 3.16. $\quad 1$. An operator $g \in \mathcal{L}\left(L^{2}\left(\mathbb{R}_{+}\right)\right)$which induces continuous operators $(3.20)$ is called a Green operator of type 0 . Let $\Gamma^{0}\left(\mathbb{R}_{+}\right)$denote the space of those operators.

2. An operator of the form $\sum_{j=0}^{d} g_{j} \partial_{t}^{j}$ for $g_{j} \in \Gamma^{0}\left(\mathbb{R}_{+}\right), d \in \mathbb{N}$, is called a Green operator of type $d$. Let $\Gamma^{d}\left(\mathbb{R}_{+}\right)$denote the space of those operators.

Remark 3.17. Any $g \in \Gamma^{d}\left(\mathbb{R}_{+}\right)$induces a compact operator

$$
g: H^{s}\left(\mathbb{R}_{+}\right) \rightarrow H^{s}\left(\mathbb{R}_{+}\right)
$$

for every $s \in \mathbb{R}, s>d-\frac{1}{2}$. Moreover $g$ induces a continuous operator

$$
g: H^{s}\left(\mathbb{R}_{+}\right) \rightarrow \mathcal{S}\left(\overline{\mathbb{R}}_{+}\right)
$$

for those $s$.

In fact, $\partial_{t}^{j}: H^{s}\left(\mathbb{R}_{+}\right) \rightarrow H^{s-j}\left(\mathbb{R}_{+}\right)$is continuous for every $j \in \mathbb{N}$ as well as and $g_{0}: H^{s-j}\left(\mathbb{R}_{+}\right) \rightarrow \mathcal{S}\left(\overline{\mathbb{R}}_{+}\right)$when $s-j>-\frac{1}{2}, g_{0} \in \Gamma^{0}\left(\mathbb{R}_{+}\right)$.

Lemma 3.18. Let $g \in \Gamma^{0}\left(\mathbb{R}_{+}\right)$, and let $1+g: L^{2}\left(\mathbb{R}_{+}\right) \rightarrow L^{2}\left(\mathbb{R}_{+}\right)$be an invertible operator. Then there is an $h \in \Gamma^{0}\left(\mathbb{R}_{+}\right)$such that $(1+g)^{-1}=1+h$. 
Proof. Let $b:=(1+g)^{-1}$ which belongs to $\mathcal{L}\left(L^{2}\left(\mathbb{R}_{+}\right)\right)$. Writing $b=1+h$ for $h:=b-1$ we obtain $(1+g)(1+h)=1$, i.e., $h+g+g h=0$. This yields $h=-g(1+h)$, and hence $h: L^{2}\left(\mathbb{R}_{+}\right) \rightarrow \mathcal{S}\left(\overline{\mathbb{R}}_{+}\right)$is continuous, cf. Definition 3.16 (i). Moreover $\left(1+g^{*}\right)\left(1+h^{*}\right)=1$ yields $g^{*}+h^{*}+g^{*} h^{*}=0$, i.e. $h^{*}=-g^{*}\left(1+h^{*}\right)$ which shows again the continuity $h^{*}: L^{2}\left(\mathbb{R}_{+}\right) \rightarrow \mathcal{S}\left(\overline{\mathbb{R}}_{+}\right)$. In other words, $h \in \Gamma^{0}\left(\mathbb{R}_{+}\right)$.

Corollary 3.19. Let $a(\tau) \in S_{\mathrm{tr}}^{\mu}(\mathbb{R}), b(\tau) \in S_{\mathrm{tr}}^{\nu}(\mathbb{R}) ;$ then

$$
\mathrm{op}^{+}(a) \mathrm{op}^{+}(b)=\mathrm{op}^{+}(a b)+g
$$

for some $g \in \Gamma^{0}\left(\mathbb{R}_{+}\right)$.

Proof. For $\mu=\nu=0$ we have

$$
\mathrm{op}^{+}(a) \mathrm{op}^{+}(b)=\mathrm{r}^{+} \mathrm{op}(a) \mathrm{e}^{+} \mathrm{r}^{+} \mathrm{op}(b) \mathrm{e}^{+}=\mathrm{r}^{+} \mathrm{op}(a) \mathrm{op}(b) \mathrm{e}^{+}+\mathrm{r}^{+} \mathrm{op}(a) \vartheta_{-} \mathrm{op}(b) \mathrm{e}^{+}
$$

for the characteristic function $\vartheta_{-}$of $\mathbb{R}_{-}$. Since

$$
\mathrm{r}^{+} \mathrm{op}(a) \vartheta_{-} \mathrm{op}(b) \mathrm{e}^{+}=\left(\mathrm{r}^{+} \mathrm{op}(a) \mathrm{e}^{-} \varepsilon^{*}\right)\left(\varepsilon^{*} \mathrm{r}^{-} \mathrm{op}(b) \mathrm{e}^{+}\right)=: g
$$

and the factors in the middle are Green operators of type zero, cf. Proposition 3.13, we obtain $g \in \Gamma^{0}\left(\mathbb{R}_{+}\right)$, since $\Gamma^{0}\left(\mathbb{R}_{+}\right)$is closed under compositions.

It remains to consider $\mu \in \mathbb{N}$ or $\nu \in \mathbb{N}$. In this case we write

$$
a(\tau)=a_{0}(\tau)+p(\tau), b(\tau)=b_{0}(\tau)+q(\tau)
$$

for $a_{0}, b_{0} \in S_{\mathrm{tr}}^{-1}(\mathbb{R})$ and polynomials $p$ and $q$ of degree $\mu$ and $\nu$, respectively. Since polynomials are minus- and plus-symbols at the same time we have

$$
\mathrm{op}^{+}(p) \mathrm{op}^{+}\left(b_{0}\right)=\mathrm{op}^{+}\left(p b_{0}\right), \mathrm{op}^{+}\left(a_{0}\right) \mathrm{op}^{+}(q)=\mathrm{op}^{+}\left(a_{0} q\right),
$$

i.e., when we define $g$ by op ${ }^{+}\left(a_{0}\right) \mathrm{op}^{+}\left(b_{0}\right)=\mathrm{op}^{+}\left(a_{0} b_{0}\right)+g$ (according to the first part of the proof) we obtain

$\mathrm{op}^{+}(a) \mathrm{op}^{+}(b)=\left(\mathrm{op}^{+}\left(a_{0}\right)+\mathrm{op}^{+}(p)\right)\left(\mathrm{op}^{+}\left(b_{0}\right)+\mathrm{op}^{+}(q)\right)=\mathrm{op}^{+}\left(\left(a_{0}+p\right)\left(b_{0}+q\right)\right)+g$.

More generally we have the following composition property.

Theorem 3.20. Let $a(\tau) \in S_{\mathrm{tr}}^{\mu}(\mathbb{R}), b(\tau) \in S_{\mathrm{tr}}^{\nu}(\mathbb{R})$, and $g \in \Gamma^{d}\left(\mathbb{R}_{+}\right), h \in \Gamma^{e}\left(\mathbb{R}_{+}\right)$. Then

$$
\left(\mathrm{op}^{+}(a)+g\right)\left(\mathrm{op}^{+}(b)+h\right)=\mathrm{op}^{+}(a b)+k
$$

for a certain $k \in \Gamma^{\max \{\nu+d, e\}}\left(\mathbb{R}_{+}\right)$.

Proof. By virtue of Corollary 3.19 it remains to discuss the compositions

$$
\mathrm{op}^{+}(a) h, g \mathrm{op}^{+}(b) \text {, and } g h \text {. }
$$

It is evident that op ${ }^{+}(a) h \in \Gamma^{e}\left(\mathbb{R}_{+}\right)$and $g h \in \Gamma^{e}\left(\mathbb{R}_{+}\right)$. For the operator in the middle we write

$$
g \mathrm{op}^{+}(b)=g\left(\mathrm{op}^{+}\left(b_{0}\right)+\mathrm{op}^{+}(p)\right)
$$


where $b_{0} \in S_{\mathrm{tr}}^{-1}(\mathbb{R})$ and $p$ is a polynomial in $\tau$ of order $\nu$ (which vanishes for $\nu \leq-1)$. It is clear that $g \mathrm{op}^{+}(p) \in \Gamma^{\nu+d}\left(\mathbb{R}_{+}\right)$. What concerns $g \mathrm{op}^{+}\left(b_{0}\right)$ it suffices to assume $g=g_{0} D_{t}^{j}$ for any $0 \leq j \leq d$. Since $\tau^{j}$ is a minus-symbol we have

$$
g \mathrm{op}^{+}\left(b_{0}\right)=g_{0} \mathrm{op}^{+}\left(b_{j}\right) \text { for } b_{j}(\tau)=\tau^{j} b_{0}(\tau) \in S_{\mathrm{tr}}^{j-1}(\mathbb{R})
$$

Thus, writing $b_{j}(\tau)=c_{j}(\tau)+q_{j-1}(\tau)$ for a polynomial $q_{j-1}(\tau)$ in $\tau$ of degree $j-1$ (when $j-1 \geq 0$ ) and some $c_{j} \in S_{\mathrm{tr}}^{-1}(\mathbb{R})$ it follows that

$$
\mathrm{gop}^{+}\left(b_{0}\right)=g_{0} \mathrm{op}^{+}\left(c_{j}\right)+g_{0} \mathrm{op}^{+}\left(q_{j-1}\right) .
$$

The second summand on the right obviously belongs to $\Gamma^{j-1}\left(\mathbb{R}_{+}\right)$for $j \geq 1$ while the first one belongs to $\Gamma^{0}\left(\mathbb{R}_{+}\right)$which follows from the continuity op ${ }^{+}\left(c_{j}\right)$ : $L^{2}\left(\mathbb{R}_{+}\right) \rightarrow L^{2}\left(\mathbb{R}_{+}\right)$and $g_{0}: L^{2}\left(\mathbb{R}_{+}\right) \rightarrow \mathcal{S}\left(\overline{\mathbb{R}}_{+}\right)$and an analogous conclusion for the adjoints.

Remark 3.21. As a special case of Theorem 3.20 for $a(\tau) \in S_{\mathrm{tr}}^{\mu}(\mathbb{R}), g \in \Gamma^{d}\left(\mathbb{R}_{+}\right)$, we obtain that $\left(\mathrm{op}^{+}(a)+g\right) \mathrm{op}^{+}\left(l_{-}^{-N}\right)=\mathrm{op}^{+}\left(a l_{-}^{-N}\right)+k$ for $k \in \Gamma^{0}\left(\mathbb{R}_{+}\right)$when $-N+d \leq 0$.

Let us now turn to $2 \times 2$ block matrices of operators with upper left corners of the form

$$
\left(\begin{array}{cc}
\mathrm{op}^{+}(a)+g_{11} & g_{12} \\
g_{21} & g_{22}
\end{array}\right): \begin{gathered}
H^{s}\left(\mathbb{R}_{+}\right) \\
\mathbb{C}
\end{gathered} \rightarrow \begin{gathered}
H^{s-\mu}\left(\mathbb{R}_{+}\right) \\
\mathbb{C}
\end{gathered}
$$

for arbitrary $a(\tau) \in S_{\mathrm{tr}}^{\mu}(\mathbb{R}), \mu \in \mathbb{Z}, g_{11} \in \Gamma^{d}\left(\mathbb{R}_{+}\right), d \in \mathbb{N}, s>d-\frac{1}{2}, g_{22} \in \mathbb{C}$,

$$
g_{21} u(t)=\sum_{l=0}^{d} g_{21, l} \partial_{t}^{l} u(t) u \in H^{s}\left(\mathbb{R}_{+}\right)
$$

for $g_{21, l} v(t):=\int_{0}^{\infty} f_{21, l}(t) v(t) d t, f_{21, l} \in \mathcal{S}\left(\overline{\mathbb{R}}_{+}\right), l=0 \ldots, d$, and

$$
g_{12} c:=c f(t), c \in \mathbb{C},
$$

for some $f \in \mathcal{S}\left(\overline{\mathbb{R}}_{+}\right)$. An operator of the form (3.24) is called a trace operator of type $d$, and (3.25) a potential operator (for the boundary symbolic calculus of operators with the transmission property at the boundary).

In a similar manner we define analogues of (3.23) where $\mathbb{C}$ on the left is replaced by $\mathbb{C}^{j_{-}}$and on the right by $\mathbb{C}^{j_{+}}$for certain $j_{-}, j_{+} \in \mathbb{N}$ (if one of the dimensions is zero then we have row or column matrices which are admitted as well). Let $\mathcal{B}^{\mu, d}\left(\overline{\mathbb{R}}_{+} ; j_{-}, j_{+}\right)$denote the space of such block matrices. Moreover, let $\mathcal{B}_{G}^{d}\left(\overline{\mathbb{R}}_{+} ; j_{-}, j_{+}\right)$be the subspace of operators $(3.23)$ defined by $a \equiv 0$.

$\Gamma^{d}\left(\mathbb{R}_{+}\right)$.

Thus $\mathcal{B}_{G}^{d}\left(\overline{\mathbb{R}}_{+} ; 0,0\right)=\Gamma^{d}\left(\mathbb{R}_{+}\right) ;$in future we also write $\mathcal{B}_{G}^{d}\left(\overline{\mathbb{R}}_{+}\right)$rather than 
Remark 3.22. More generally we have $\mathcal{B}^{\mu, d}\left(\overline{\mathbb{R}}_{+} ; \boldsymbol{v}\right)$ for $\boldsymbol{v}=\left(k, l ; j_{-}, j_{+}\right)$, defined to be the space of $2 \times 2$ block matrices where the upper left corner itself is an $l \times k$ matrix of operators as in the upper left corner of $(3.23)$, while $g_{12}$ is a $j_{-} \times k$ matrix of potential operators, etc. For every fixed $\mu \in \mathbb{Z}, d \in \mathbb{N}$, the space of such matrices is a (nuclear) Fréchet space in a natural way. The future homogeneous boundary symbols of BVPs are symbols in $(y, \eta)$ with values in such spaces.

Remark 3.23. It can easily be proved, cf. [18, Proposition 4.1.46], that every $g \in$ $\Gamma^{d}\left(\mathbb{R}_{+}\right)$for $d>0$ has a unique representation

$$
g=g_{0}+\sum_{j=0}^{d-1} k_{j} \circ \mathrm{r}^{\prime} D_{t}^{j}
$$

for $g_{0} \in \Gamma^{0}\left(\mathbb{R}_{+}\right)$, potential operators $k_{j}$, and $\mathrm{r}^{\prime} u:=u(0)$. Similarly, a trace operator $b$ of type $d>0$ can uniquely be written as

$$
b=b_{0}+\sum_{j=0}^{d-1} c_{j} \circ \mathrm{r}^{\prime} D_{t}^{j}
$$

for a trace operator $b_{0}$ of type 0 and constants $c_{j}$.

Example. The operator

$$
\left(\begin{array}{c}
\mathrm{op}^{+}\left(-|\eta|^{2}-\tau^{2}\right) \\
\mathrm{r}^{\prime} D_{t}^{k}
\end{array}\right): H^{s}\left(\mathbb{R}_{+}\right) \rightarrow \stackrel{H^{s-2}\left(\mathbb{R}_{+}\right)}{\oplus}
$$

$\mathrm{r}^{\prime} v=\left.v\right|_{t=0}$, belongs to $\mathcal{B}^{2, k+1}\left(\overline{\mathbb{R}}_{+} ; 0,1\right), k \in \mathbb{N}$, and (3.26) is an isomorphism for every $\eta \in \mathbb{R}^{n-1} \backslash\{0\}, s>\max \left\{\frac{3}{2}, k+\frac{1}{2}\right\}$. The operator family (3.26) for $k=0$ is just the boundary symbol of the Dirichlet problem for the Laplace equation and for $k=1$ of the Neumann problem.

The inverse of (3.26) for $k=0$ is explicitly computed in [12, Section 3.3.4]. Setting $a(\eta, \tau):=-|\eta|^{2}-|\tau|^{2}$ the result is

$$
\left(\begin{array}{c}
\mathrm{op}^{+}(a)(\eta) \\
\mathrm{r}^{\prime}
\end{array}\right)^{-1}=\left(-\mathrm{op}^{+}\left(l_{+}^{-1}\right)(\eta) \mathrm{op}^{+}\left(l_{-}^{-1}\right)(\eta) d(\eta)\right)
$$

for $l_{ \pm}(\eta):=|\eta| \pm i \tau$ and a potential operator $d(\eta)$ defined by $d(\eta): c \rightarrow c e^{-|\eta| t}$, $c \in \mathbb{C}$. By virtue of Corollary 3.19 we have

$$
-\mathrm{op}^{+}\left(l_{+}^{-1}\right)(\eta) \mathrm{op}^{+}\left(l_{-}^{-1}\right)(\eta)=\mathrm{op}^{+}\left(a^{-1}\right)(\eta)+g(\eta)
$$

for a Green operator family $g(\eta)$ of type 0 . Note that $g(\eta)$ is just the homogeneous boundary symbol of the well-known Green's function of the Dirichlet problem for the Laplacian (twisted homogeneous of order -2 ).

It is now easy also to compute the inverses of (3.26) for arbitrary $k \in \mathbb{N}$, especially, of the boundary symbol of the Neumann problem. In fact, similarly as 
(2.19), now on the level of boundary symbols, we have

$$
\left(\begin{array}{c}
\mathrm{op}^{+}(a)(\eta) \\
\mathrm{r}^{\prime} D_{t}^{k}
\end{array}\right)(p(\eta) \quad d(\eta))=\left(\begin{array}{cc}
1 & 0 \\
b(\eta) & q_{k}(\eta)
\end{array}\right)
$$

for $p(\eta):=\mathrm{op}^{+}\left(a^{-1}\right)(\eta)+g(\eta), b(\eta):=\mathrm{r}^{\prime} D_{t}^{k}\left(\mathrm{op}^{+}\left(a^{-1}\right)(\eta)+g(\eta)\right), g_{k}(\eta):=$ $\mathrm{r}^{\prime} D_{t}^{k} d(\eta)$. We have

$$
\mathrm{r}^{\prime} D_{t}^{k} d(\eta)=\left.D_{t}^{k} e^{-|\eta| t}\right|_{t=0}=(i|\eta|)^{k}
$$

which is just the homogeneous principal symbol of the elliptic operator $T_{k} K_{0} \in$ $L_{\mathrm{cl}}^{k}(Y)$ occurring in the lower right corner of the operator (3.27). Thus

$$
\begin{aligned}
& \left(\begin{array}{c}
\mathrm{op}^{+}(a)(\eta) \\
\mathrm{r}^{\prime} D_{t}^{k}
\end{array}\right)^{-1}=(p(\eta) \quad d(\eta))\left(\begin{array}{cc}
1 & 0 \\
b(\eta) & q_{k}(\eta)
\end{array}\right)^{-1} \\
& =\left(p(\eta)-d(\eta) q_{k}^{-1}(\eta) b(\eta) \quad d(\eta) q^{-1}(\eta)\right) .
\end{aligned}
$$
low.

General compositions of boundary symbols are studied in Theorem 3.26 be-

Remark 3.24. It is interesting to consider elliptic boundary value problems for the elliptic operator $T_{k} K_{0}$ on a smooth submanifold of $Y$ with boundary $Z$. This makes sense, for instance, when we reduce the Zaremba problem for $\Delta$ (defined by jumping conditions from Dirichlet to Neumann along $Z$ ) to $Y$. Then a basic difficulty is that $T_{k} K_{0}$ fails to have the transmission property at $Z$, cf. Definition 4.11 below, unless $k$ is even. Mixed problems (i.e., with jumping boundary conditions) belong to the motivation to study BVPs for operators without the transmission property. Another (possibly even stronger) motivation is the similarity between mixed and (specific) edge problems.

Theorem 3.25. We have

$$
\begin{gathered}
\boldsymbol{a} \in \mathcal{B}^{\mu, d}\left(\overline{\mathbb{R}}_{+} ; j_{0}, j_{+}\right), \boldsymbol{b} \in \mathcal{B}^{\nu, e}\left(\overline{\mathbb{R}}_{+} ; j_{-}, j_{0}\right) \Rightarrow \boldsymbol{a} \boldsymbol{b} \in \mathcal{B}^{\mu+\nu, \max \{\nu+d, e\}}\left(\overline{\mathbb{R}}_{+} ; j_{-}, j_{+}\right), \\
\text {and }(\boldsymbol{a}, \boldsymbol{b}) \rightarrow \boldsymbol{a b} \text { defines a bilinear continuous map } \\
\quad \mathcal{B}^{\mu, d}\left(\overline{\mathbb{R}}_{+} ; j_{0}, j_{+}\right) \times \mathcal{B}^{\nu, e}\left(\overline{\mathbb{R}}_{+} ; j_{-}, j_{0}\right) \rightarrow \mathcal{B}^{\mu+\nu, \max \{\nu+d, e\}}\left(\overline{\mathbb{R}}_{+} ; j_{-}, j_{+}\right)
\end{gathered}
$$

between the respective Fréchet spaces.

Proof. The result for the composition of upper left corners is contained in Theorem 3.20. The proof for the remaining entries is straightforward and left to the reader.

Theorem 3.26. Let $\boldsymbol{a} \in \mathcal{B}^{0,0}\left(\overline{\mathbb{R}}_{+} ; j_{-}, j_{+}\right)$, and define the adjoint $\boldsymbol{a}^{*}$ by

$$
(\boldsymbol{a} u, v)_{L^{2}\left(\mathbb{R}_{+}\right) \oplus \mathbb{C}^{j_{+}}}=\left(u, \boldsymbol{a}^{*} v\right)_{L^{2}\left(\mathbb{R}_{+}\right) \oplus \mathbb{C}^{j_{-}}}
$$

for all $u \in L^{2}\left(\mathbb{R}_{+}\right) \oplus \mathbb{C}^{j_{-}}, v \in L^{2}\left(\mathbb{R}_{+}\right) \oplus \mathbb{C}^{j_{+}}$. Then we have $\boldsymbol{a}^{*} \in \mathcal{B}^{0,0}\left(\overline{\mathbb{R}}_{+} ; j_{+}, j_{-}\right)$, and $\boldsymbol{a} \rightarrow \boldsymbol{a}^{*}$ defines an (antilinear), continuous map

$$
\mathcal{B}^{0,0}\left(\overline{\mathbb{R}}_{+} ; j_{-}, j_{+}\right) \rightarrow \mathcal{B}^{0,0}\left(\overline{\mathbb{R}}_{+} ; j_{+}, j_{-}\right) .
$$


Proof. The result for the upper left corner follows from Proposition 3.12, together with Corollary 3.14. The proof for the remaining entries is straightforward and left to the reader.

Definition 3.27. A symbol $a(\tau) \in S_{\mathrm{tr}}^{\mu}(\mathbb{R})$ is called elliptic (of order $\mu$ ) if $a(\tau) \neq 0$ for all $\tau \in \mathbb{R}$, and if $a_{0}\left(=a_{0}^{-}=a_{0}^{+}\right.$) does not vanish (cf. the notation in (3.8)). Moreover, we call an $\boldsymbol{a} \in \mathcal{B}^{\mu, d}\left(\overline{\mathbb{R}}_{+} ; j_{-}, j_{+}\right)$elliptic if the symbol $a(\tau) \in S_{\mathrm{tr}}^{\mu}(\mathbb{R})$ in the upper left corner of (3.23) is elliptic.

Theorem 3.28. Let $a(\tau) \in S_{\mathrm{tr}}^{\mu}(\mathbb{R})$ be elliptic, and $g \in \Gamma^{d}\left(\mathbb{R}_{+}\right)$; then

$$
\boldsymbol{a}:=\mathrm{op}^{+}(a)+g: H^{s}\left(\mathbb{R}_{+}\right) \rightarrow H^{s-\mu}\left(\mathbb{R}_{+}\right)
$$

is a Fredholm operator for every $s>\max \{\mu, d\}-\frac{1}{2}$, and $\boldsymbol{p}:=\mathrm{op}^{+}\left(a^{-1}\right)$ is a parametrix of $\boldsymbol{a}$.

Proof. Because of the assumption on $s$ the operator

$$
\text { op }^{+}\left(a^{-1}\right): H^{s-\mu}\left(\mathbb{R}_{+}\right) \rightarrow H^{s}\left(\mathbb{R}_{+}\right)
$$

is continuous. From Corollary 3.19 and Theorem 3.20 we have

$$
\mathrm{op}^{+}\left(a^{-1}\right)\left\{\mathrm{op}^{+}(a)+g\right\}=\mathrm{op}^{+}\left(a a^{-1}\right)+k=1+k
$$

where $k=h+$ op $^{+}\left(a^{-1}\right) g$ for an $h \in \Gamma^{0}\left(\mathbb{R}_{+}\right)$and op ${ }^{+}\left(a^{-1}\right) g \in \Gamma^{d}\left(\mathbb{R}_{+}\right)$. Thus since

$$
k: H^{s}\left(\mathbb{R}_{+}\right) \rightarrow H^{s}\left(\mathbb{R}_{+}\right)
$$

is compact for $s>d-\frac{1}{2}$, cf. Remark 3.17, the operator op ${ }^{+}\left(a^{-1}\right)$ is a left parametrix. In a similar manner we obtain that op ${ }^{+}\left(a^{-1}\right)$ is a right parametrix. In fact, we have to compute

$$
\left\{\mathrm{op}^{+}(a)+g\right\} \mathrm{op}^{+}\left(a^{-1}\right)=\mathrm{op}^{+}\left(a a^{-1}\right)+k=1+k
$$

where $k=h+g \mathrm{op}^{+}\left(a^{-1}\right)$, for a $h \in \Gamma^{0}\left(\mathbb{R}_{+}\right)$, and $g \mathrm{op}^{+}\left(a^{-1}\right) \in \Gamma^{\max \{-\mu+d, 0\}}\left(\mathbb{R}_{+}\right)$.

This can be applied to functions in $H^{s-\mu}\left(\mathbb{R}_{+}\right)$when $s$ satisfies the conditions $s-\mu>-\frac{1}{2}$ and $s-\mu>\max \{-\mu+d, 0\}-\frac{1}{2}$. In the case $\max \{-\mu+d, 0\}=0$ the latter is the same as the first condition while for $\max \{-\mu+d, 0\}=-\mu+d \geq 0$ the condition is $s-\mu>-\mu+d-\frac{1}{2}$, i.e., $s>d-\frac{1}{2}$. For $s$ it follows altogether $s>\max \{\mu, d\}-\frac{1}{2}$, and we can apply again Remark 3.17 .

Theorem 3.29. Let $a(\tau) \in S_{\mathrm{tr}}^{\mu}(\mathbb{R})$ be elliptic, and $g \in \Gamma^{d}\left(\mathbb{R}_{+}\right)$. Then $V:=\operatorname{ker}\left(\mathrm{op}^{+}(a)+\right.$ $g)$ is a finite-dimensional subspace of $\mathcal{S}\left(\overline{\mathbb{R}}_{+}\right)$, and there is a finite-dimensional subspace $W \subset \mathcal{S}\left(\overline{\mathbb{R}}_{+}\right)$such that

$$
\operatorname{im}\left(\mathrm{op}^{+}(a)+g\right)+W=H^{s-\mu}\left(\mathbb{R}_{+}\right) .
$$

This is true for all real $s>\max \{\mu, d\}-\frac{1}{2}$ with the same spaces $V$ and $W$. It follows that ind $\left(\mathrm{op}^{+}(a)+g\right)$ is independent of $s$. 
Proof. Let us set $\boldsymbol{a}:=\mathrm{op}^{+}(a)+g$ and assume $u \in H^{s}\left(\mathbb{R}_{+}\right), \boldsymbol{a} u=0$. Then from the relation (3.30) it follows that $(1+k) u=0$, i.e., $u=-k u$, which implies $u \in \mathcal{S}\left(\overline{\mathbb{R}}_{+}\right)$, cf. the formula (3.21). In other words, $V=\operatorname{ker} \boldsymbol{a} \subseteq \operatorname{ker}(1+k)$ is a finite-dimensional subspace of $\mathcal{S}\left(\overline{\mathbb{R}}_{+}\right)$, independent of $s=\max \{\mu, d\}-\frac{1}{2}$. In the case $d=0, \mu \leq 0$ we can do the same for the formal adjoint $\boldsymbol{a}^{*}$, and we may set $W=\operatorname{ker} \boldsymbol{a}^{*}$ which is a finite-dimensional subspace of $\mathcal{S}\left(\overline{\mathbb{R}}_{+}\right)$independent of $s$.

To find $W$ in general we set $\boldsymbol{a}:=\mathrm{op}^{+}(a)+g$ which we check as an operator $\boldsymbol{a}: H^{s}\left(\mathbb{R}_{+}\right) \rightarrow H^{s-\mu}\left(\mathbb{R}_{+}\right)$for $s>\max \{\mu, d\}-\frac{1}{2}$. Let us set $\boldsymbol{l}_{-}^{N}:=$ op $^{+}\left(l_{-}^{N}\right)$ for any $N \in \mathbb{Z}$. In particular, for $N:=\max \{\mu, d\}$ we have

$$
\boldsymbol{a}=\boldsymbol{a} \boldsymbol{l}_{-}^{N}=\boldsymbol{a}_{0} \boldsymbol{l}_{-}^{N}: H^{s}\left(\mathbb{R}_{+}\right) \rightarrow H^{s-\mu}\left(\mathbb{R}_{+}\right)
$$

where $\boldsymbol{a}_{0}=\operatorname{op}^{+}\left(a l_{-}^{-N}\right)+k$ for some $k \in \Gamma^{0}\left(\mathbb{R}_{+}\right)$, i.e., $\boldsymbol{a}_{0} \in \mathcal{B}^{\nu, 0}\left(\overline{\mathbb{R}}_{+}\right)$for $\nu=$ $\mu-N \leq 0$. Then $\boldsymbol{a}$ can be regarded as a chain of operators

$$
\boldsymbol{a}: H^{s}\left(\mathbb{R}_{+}\right) \rightarrow H^{s-N}\left(\mathbb{R}_{+}\right) \rightarrow H^{s-N-\nu}\left(\mathbb{R}_{+}\right)=H^{s-\mu}\left(\mathbb{R}_{+}\right)
$$

where the first one, namely, $\boldsymbol{l}_{-}^{N}$ is an isomorphism where $s-N \geq-\frac{1}{2}$, and the second one $\boldsymbol{a}_{0}$ is elliptic of order $\nu$. For the latter we apply the first part of the proof, i.e., we find a finite-dimensional $W \subset \mathcal{S}\left(\overline{\mathbb{R}}_{+}\right)$such that ima $\boldsymbol{a}_{0}+W=H^{s-\mu}\left(\mathbb{R}_{+}\right)$. This entails im $\boldsymbol{a}+W=H^{s-\mu}\left(\mathbb{R}_{+}\right)$, since $\boldsymbol{a}=\boldsymbol{a}_{0} \boldsymbol{l}_{-}^{N}$.

Proposition 3.30. Let $\boldsymbol{a}=\mathrm{op}^{+}(a)+g \in \mathcal{B}^{\mu, d}\left(\overline{\mathbb{R}}_{+}\right)$where $a(\tau) \in S_{\mathrm{tr}}^{\mu}(\mathbb{R})$ is elliptic of order $\mu$. Moreover, let $W \subset \mathcal{S}\left(\overline{\mathbb{R}}_{+}\right)$be a finite-dimensional subspace, and $k$ : $\mathbb{C}^{j} \rightarrow W$ a linear map. Then

$$
\left(\begin{array}{l}
u \\
c
\end{array}\right) \in \underset{\mathbb{C}^{j}}{\bigoplus^{s}\left(\mathbb{R}_{+}\right)}, \quad \boldsymbol{a} u+k c=0
$$

for any $s>\max \{\mu, d\}-\frac{1}{2}$ implies $u \in \mathcal{S}\left(\overline{\mathbb{R}}_{+}\right)$, and the space of all solutions of (3.33) is a finite-dimensional subspace of $H^{s}\left(\mathbb{R}_{+}\right) \oplus \mathbb{C}^{j}$, independent of $s$.

Proof. First observe that $(\boldsymbol{a} \quad k)$ is a Fredholm operator

$$
\left(\begin{array}{lll}
\text { a } & k
\end{array}\right): \stackrel{\bigoplus^{s}\left(\mathbb{R}_{+}\right)}{\bigoplus_{\mathbb{C}^{j}}} \rightarrow H^{s-\mu}\left(\mathbb{R}_{+}\right) .
$$

Then, analogously as in the proof of Theorem 3.29 we pass to the operator

$$
\left(\begin{array}{l}
\boldsymbol{p} \\
0
\end{array}\right)\left(\begin{array}{ll}
\boldsymbol{a} & k
\end{array}\right)=\left(\begin{array}{cc}
\boldsymbol{p a} & \boldsymbol{p} k \\
0 & 0
\end{array}\right): \begin{gathered}
H^{s}\left(\mathbb{R}_{+}\right) \\
\mathbb{C}^{j}
\end{gathered} \rightarrow \begin{gathered}
H^{s}\left(\mathbb{R}_{+}\right) \\
\mathbb{C}^{j}
\end{gathered}
$$

for $\boldsymbol{p}:=\mathrm{op}^{+}\left(a^{-1}\right)$. The composition $l:=\boldsymbol{p} k$ is a potential operator, and we have $\boldsymbol{p a}=1+h$ for an operator $h \in \Gamma^{d}\left(\mathbb{R}_{+}\right)$. The kernel of $(3.34)$ is contained in the kernel of $\left(\begin{array}{lll}1+h & l\end{array}\right)$. The kernel of $\left(\begin{array}{ll}1+h & l\end{array}\right)$ consists of all ${ }^{\mathrm{t}}\left(\begin{array}{ll}u & c\end{array}\right)$ such that $(1+h) u+l c=0$, i.e., $u=-h u+l c \in \mathcal{S}\left(\overline{\mathbb{R}}_{+}\right)$. 
Proposition 3.31. Let op ${ }^{+}(a)+g \in \mathcal{B}^{\mu, d}\left(\overline{\mathbb{R}}_{+}\right)$be elliptic of order $\mu$. Then there exists a $2 \times 2$ block matrix operator

$$
\boldsymbol{a}=\left(\begin{array}{cc}
\mathrm{op}^{+}(a)+g & k \\
b & q
\end{array}\right): \begin{gathered}
H^{s}\left(\mathbb{R}_{+}\right) \\
\mathbb{C}^{j_{-}}
\end{gathered} \rightarrow \begin{gathered}
H^{s-\mu}\left(\mathbb{R}_{+}\right) \\
\mathbb{C}^{j_{+}}
\end{gathered}
$$

for a trace operator $b$, a potential operator $k$ and $a j_{+} \times j_{-}$matrix $q$, such that (3.35) is an isomorphism for all $s>\max \{\mu, d\}-\frac{1}{2}$, and we have

$$
\operatorname{ind}\left(\mathrm{op}^{+}(a)+g\right)=\operatorname{ind~op}^{+}(a)=j_{+}-j_{-} .
$$

The operator (3.35) is an isomorphism if and only if

$$
\begin{aligned}
& \mathcal{S}\left(\overline{\mathbb{R}}_{+}\right) \quad \mathcal{S}\left(\overline{\mathbb{R}}_{+}\right) \\
& \text {a: } \underset{\mathbb{C}^{j_{-}}}{\oplus} \rightarrow \underset{\mathbb{C}^{j_{+}}}{\oplus}
\end{aligned}
$$

is an isomorphism.

Proof. Applying Theorem 3.29 we find a finite-dimensional subspace $W \subset \mathcal{S}\left(\overline{\mathbb{R}}_{+}\right)$ such that (3.31) holds for all $s>\max \{\mu, d\}-\frac{1}{2}$. Choose any $j_{-} \in \mathbb{N}, j_{-} \geq \operatorname{dim} W$, and a linear surjective map $k: \mathbb{C}^{j} \rightarrow W$. Then

$$
\left(\mathrm{op}^{+}(a)+g \quad k\right): \stackrel{H^{s}\left(\mathbb{R}_{+}\right)}{\underset{\mathbb{C}^{j_{-}}}{\oplus}} \rightarrow H^{s-\mu}\left(\mathbb{R}_{+}\right)
$$

is obvisously surjective for all $s$. By virtue of Proposition 3.30 its kernel $V$ is a subspace of ${ }^{\mathrm{t}}\left(\mathcal{S}\left(\overline{\mathbb{R}}_{+}\right) \oplus \mathbb{C}^{j-}\right)$ of finite dimension $j_{+}$. Choosing an isomorphism

$$
\left(\begin{array}{ll}
b & q
\end{array}\right): V \rightarrow \mathbb{C}^{j_{+}}
$$

it suffices to extend $b$ to a trace operator $b: H^{s}\left(\mathbb{R}_{+}\right) \rightarrow \mathbb{C}^{j_{+}}$(for simplicity denoted by the same letter). Then, according to Lemma 2.3 we obtain an isomorphism (3.35).

Theorem 3.32. Let $\boldsymbol{a} \in \mathcal{B}^{\mu, d}\left(\overline{\mathbb{R}}_{+} ; j_{-}, j_{+}\right)$be given as in (3.35), let the upper left corner be elliptic in the sense of Definition 3.27, and assume that $\boldsymbol{a}$ defines an isomorphism (3.37). Then we have $\boldsymbol{a}^{-1} \in \mathcal{B}^{-\mu,(d-\mu)^{+}}\left(\overline{\mathbb{R}}_{+} ; j_{+}, j_{-}\right)$where $\nu^{+}:=$ $\max \{\nu, 0\}$.

Proof. By virtue of Theorem 3.28 the operator (3.29) is Fredholm where op ${ }^{+}\left(a^{-1}\right)$ is a parametrix. According to Proposition 3.31 there is a $2 \times 2$ block matrix isomorphism of the form

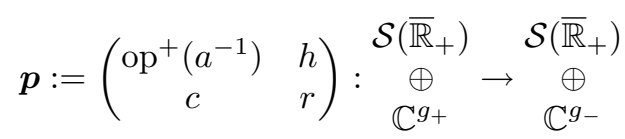


for a suitable trace operator $c$ of type 0 and a potential operator $h$. Since op ${ }^{+}\left(a^{-1}\right)$ is a parametrix of op ${ }^{+}(a)$, cf. Theorem 3.28, we have ind op ${ }^{+}\left(a^{-1}\right)=-$ ind op $^{+}(a)=$ $j_{-}-j_{+}$and from $(3.36)$

$$
\text { ind op }{ }^{+}\left(a^{-1}\right)=g_{-}-g_{+}=j_{-}-j_{+} \text {. }
$$

In the case $N:=g_{-}-j_{-} \in \mathbb{N}$ which implies $g_{+}-j_{+}=N$ we pass from $\boldsymbol{a}$ to $\boldsymbol{a} \oplus \mathrm{id}_{\mathbb{C}^{N}}$ which is again an isomorphism with $\left(j_{-}, j_{+}\right)$replaced by $\left(g_{-}, g_{+}\right)$. On the other hand when $N:=j_{-}-g_{-} \in \mathbb{N}$ where $j_{+}-g_{+}=N$, from $\boldsymbol{p}$ we pass to $\boldsymbol{p} \oplus \mathrm{id}_{\mathbb{C}^{N}}$ which is an isomorphism with $\left(g_{-}, g_{+}\right)$replaced by $\left(j_{-}, j_{+}\right)$. In any case, to find $\boldsymbol{a}^{-1}$ it suffices to assume that $j_{-}=g_{-}, j_{+}=g_{+}$. Now the composition $\boldsymbol{a p}$ is of the form

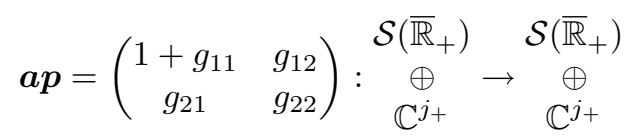

for a $\boldsymbol{g}=\left(g_{i j}\right)_{i, j=1,2} \in \mathcal{B}_{G}^{(d-\mu)^{+}}\left(\overline{\mathbb{R}}_{+} ; j_{+}, j_{+}\right)$. By virtue of Lemma 3.33 below we have

$$
\left(\left(\begin{array}{ll}
1 & 0 \\
0 & 0
\end{array}\right)+\boldsymbol{g}\right)^{-1}=\left(\begin{array}{ll}
1 & 0 \\
0 & 0
\end{array}\right)+\boldsymbol{l}
$$

for an $\boldsymbol{l} \in \mathcal{B}_{G}^{(d-\mu)^{+}}\left(\overline{\mathbb{R}}_{+} ; j_{+}, j_{+}\right)$. Then Theorem 3.25 gives us

$$
\boldsymbol{a}^{-1}=\boldsymbol{p}\left(\left(\begin{array}{ll}
1 & 0 \\
0 & 0
\end{array}\right)+\boldsymbol{l}\right) \in \mathcal{B}^{-\mu,(d-\mu)^{+}}\left(\overline{\mathbb{R}}_{+} ; j_{+}, j_{-}\right) \text {. }
$$

Lemma 3.33. Let $\boldsymbol{g} \in \mathcal{B}_{G}^{d}\left(\overline{\mathbb{R}}_{+} ; j, j\right)$, and assume that

$$
\left(\begin{array}{ll}
1 & 0 \\
0 & 0
\end{array}\right)+\boldsymbol{g}: \quad \stackrel{\bigoplus^{s}\left(\mathbb{R}_{+}\right)}{\mathbb{C}^{j}} \rightarrow \stackrel{\bigoplus^{s}\left(\mathbb{R}_{+}\right)}{\bigoplus^{j}}
$$

is invertible for any $s>d-\frac{1}{2}$. Then the inverse of (3.39) has the form $\left(\begin{array}{ll}1 & 0 \\ 0 & 0\end{array}\right)+\boldsymbol{l}$ for some $\boldsymbol{l} \in \mathcal{B}_{G}^{d}\left(\overline{\mathbb{R}}_{+} ; j, j\right)$.

Proof. For convenience we set $\boldsymbol{g}=\left(\begin{array}{ll}G & K \\ T & Q\end{array}\right)$. Then, in particular, $Q$ is a $j \times j$ matrix. Since isomorphisms in a Hilbert space form an open set, a small perturbation of $Q$ allows us to pass to an invertible operator $\left(\begin{array}{cc}1+G & K \\ T & R\end{array}\right)$ where $R$ is an invertible $j \times j$ matrix. Assume that we have computed $\left(\begin{array}{cc}1+G & K \\ T & R\end{array}\right)^{-1}$. Then we have $\left(\begin{array}{cc}1+G & K \\ T & R\end{array}\right)^{-1}\left(\begin{array}{cc}1+G & K \\ T & Q\end{array}\right)=\left(\begin{array}{cc}1 & 0 \\ D & J\end{array}\right)$ which is again invertible; this 
entails the invertibility of $J$. We obtain

$$
\left(\begin{array}{cc}
1+G & K \\
T & Q
\end{array}\right)^{-1}=\left(\begin{array}{cc}
1 & 0 \\
-J^{-1} D & J^{-1}
\end{array}\right)\left(\begin{array}{cc}
1+G & K \\
T & R
\end{array}\right)^{-1}
$$

Thus it remains to characterise the second factor on the right of (3.40). The identity

$$
\left(\begin{array}{cc}
1 & -K R^{-1} \\
0 & 1
\end{array}\right)\left(\begin{array}{cc}
1+G & K \\
T & R
\end{array}\right)\left(\begin{array}{cc}
1 & 0 \\
-R^{-1} T & R^{-1}
\end{array}\right)=\left(\begin{array}{cc}
1+C & 0 \\
0 & 1
\end{array}\right)
$$

for $C:=G-K R^{-1} T$ shows that the operator $1+C$ is invertible, and it follows that

$$
\left(\begin{array}{cc}
1+G & K \\
T & R
\end{array}\right)^{-1}=\left(\begin{array}{cc}
1 & 0 \\
-R^{-1} T & R^{-1}
\end{array}\right)\left(\begin{array}{cc}
(1+C)^{-1} & 0 \\
0 & 1
\end{array}\right)\left(\begin{array}{cc}
1 & -K R^{-1} \\
0 & 1
\end{array}\right) .
$$

This reduces the task to the computation of $(1+C)^{-1}$.

The operator $C \in \mathcal{B}_{G}^{d}\left(\overline{\mathbb{R}}_{+}\right)$can be written in the form $C=C_{0}+\sum_{j=0}^{d-1} K_{j} T_{j}$ for a $C_{0} \in \mathcal{B}_{G}^{0}\left(\overline{\mathbb{R}}_{+}\right)$, potential operators $K_{j}$ and trace operators $T_{j}:=\mathrm{r}^{\prime} D_{t}^{j}$, cf. Remark 3.23. Since $C_{0}$ is compact in Sobolev spaces, we have $\operatorname{ind}\left(1+C_{0}\right)=0$. Because of the nature of $V:=\operatorname{ker}\left(1+C_{0}\right)$ and $W=\operatorname{coker}\left(1+C_{0}\right)$ (which are of the same dimension $l$ ) there is a trace operator $B$ of type 0 and a potential operator $D$ which induces isomorphisms

$$
B={ }^{\mathrm{t}}\left(B_{1}, \ldots, B_{l}\right): V \rightarrow \mathbb{C}^{l}, \quad D=\left(D_{1}, \ldots, D_{l}\right): \mathbb{C}^{l} \rightarrow W
$$

such that

$$
1+C_{0}+D B: H^{s}\left(\mathbb{R}_{+}\right) \rightarrow H^{s}\left(\mathbb{R}_{+}\right)
$$

is an isomorphism. Note that $C_{1}:=C_{0}+D B \in \mathcal{B}_{G}^{0}\left(\overline{\mathbb{R}}_{+}\right)$. We obtain

$$
1+C=1+C_{1}-\sum_{k=1}^{d+l} D_{k} B_{k}
$$

for $D_{l+j+1}=-K_{j}, B_{l+j+1}=T_{j}$ for $j=0, \ldots, d-1$. Now we employ the fact that there is a $C_{2} \in \mathcal{B}_{G}^{0}\left(\overline{\mathbb{R}}_{+}\right)$such that $1+C_{2}=\left(1+C_{1}\right)^{-1}$, say, as an operator $L^{2}\left(\mathbb{R}_{+}\right) \rightarrow L^{2}\left(\mathbb{R}_{+}\right)$, cf. Lemma 3.18 . In order to characterise $(1+C)^{-1}$ we form

$$
\left(1+C_{2}\right)(1+C)=1+\left(1+C_{2}\right) \sum_{k=1}^{d+l} D_{k} B_{k}=1+\sum_{k=1}^{d+l} M_{k} B_{k}=1+\mathcal{M B}
$$

for $M_{k}=\left(1+C_{2}\right) D_{k}, \mathcal{M}:=\left(M_{1}, \ldots, M_{d+l}\right), \mathcal{B}:={ }^{\mathrm{t}}\left(B_{1}, \ldots, B_{d+l}\right)$. This reduces the task to invert the operator $1+C$ to the inversion of

$$
1+\mathcal{M B}: H^{s}\left(\mathbb{R}_{+}\right) \rightarrow H^{s}\left(\mathbb{R}_{+}\right) .
$$

With the operators $\mathcal{M}$ and $\mathcal{B}$ we can also associate the operator

$$
1+\mathcal{B M}: \mathbb{C}^{l+d} \rightarrow \mathbb{C}^{l+d}, 1:=\mathrm{id}_{\mathbb{C}^{l+d}}
$$


Now we verify that $1+\mathcal{M B}$ is invertible if and only if $1+\mathcal{B} \mathcal{M}$ is invertible. In fact, setting

$$
\mathfrak{M}:=\left(\begin{array}{cc}
1 & \mathcal{M} \\
0 & 1
\end{array}\right), \mathfrak{B}:=\left(\begin{array}{cc}
1 & 0 \\
-\mathcal{B} & 1
\end{array}\right), \mathfrak{F}:=\left(\begin{array}{cc}
1 & -\mathcal{M} \\
\mathcal{B} & 1
\end{array}\right)
$$

it follows that

$$
\mathfrak{M F B}=\left(\begin{array}{cc}
1+\mathcal{M B} & 0 \\
0 & 1
\end{array}\right), \mathfrak{B F M}=\left(\begin{array}{cc}
1 & 0 \\
0 & 1+\mathcal{B M}
\end{array}\right)
$$

which gives us the desired equivalence. At the same time we see that

$$
(1+\mathcal{M B})^{-1}=1-\mathcal{M}(1+\mathcal{B} \mathcal{M})^{-1} \mathcal{B}
$$

which is of the form $1+G_{1}$ for a $G_{1} \in \mathcal{B}_{G}^{d}\left(\overline{\mathbb{R}}_{+}\right)$. Thus

$$
(1+C)^{-1}=\left(1+C_{1}\right)\left(1+G_{1}\right)=1+C_{1}+G_{1}+C_{1} G_{1}
$$

where $C_{1}+G_{1}+C_{1} G_{1} \in \mathcal{B}_{G}^{d}\left(\overline{\mathbb{R}}_{+}\right)$.

Remark 3.34. Theorem 3.32 easily extends to $\mathcal{B}^{\mu, d}\left(\overline{\mathbb{R}}_{+} ;\left(k, k ; j_{-}, j_{+}\right)\right)$for arbitrary $k, j_{-}, j_{+} \in \mathbb{N}$ (cf. Remark 3.22). The technique for the proof which mainly employs compositions of some operators also shows that the inverse continuously depends on the given operator $\boldsymbol{a}$.

\section{Pseudo-differential boundary value problems}

We develop basics on pseudo-differential BVPs with the transmission property at the boundary. Other material may be found in the author's joint monographs with Rempel [15], with Kapanadze [12], or with Harutyunyan [10], and in the monograph of Grubb [8]. The ideas here are related to the calculus on manifolds with edges. Let us first consider operators in local coordinates $x=(y, t) \in \mathbb{R}^{n-1} \times \overline{\mathbb{R}}_{+}$. The operator convention refers to the embedding of $\overline{\mathbb{R}}_{+}^{n}$ into the ambient space $\mathbb{R}^{n}$. Therefore, we first look at operators

$$
\mathrm{Op}_{x}(p) u(x)=\iint e^{i\left(x-x^{\prime}\right) \xi} p(x, \xi) u\left(x^{\prime}\right) d x^{\prime} d \xi
$$

Here $p$ belongs to Hörmander's symbol classes. Let $S^{\mu}\left(U \times \mathbb{R}^{n}\right)$ for $\mu \in \mathbb{R}$ and $U \subseteq \mathbb{R}^{m}$ open denote the set of all $p \in C^{\infty}\left(U \times \mathbb{R}^{n}\right)$ such that

$$
\left|D_{x}^{\alpha} D_{\xi}^{\beta} p(x, \xi)\right| \leq c\langle\xi\rangle^{\mu-|\beta|}
$$

for all $(x, \xi) \in K \times \mathbb{R}^{n}, K \Subset U$, and all $\alpha \in \mathbb{N}^{m}, \beta \in \mathbb{N}^{n}$, for constants $c=c(\alpha, \beta, K)>0$. We will freeley employ various standard properties such as asymptotic expansions, etc., developed in textbooks on pseudo-differential operators. The subspace $S_{\mathrm{cl}}^{\mu}\left(U \times \mathbb{R}^{n}\right)$ of classical symbols is defined by asymptotic expansions

$$
p(x, \xi) \sim \sum_{j=0}^{\infty} \chi(\xi) p_{(\mu-j)}(x, \xi)
$$


where $p_{(\mu-j)}(x, \xi) \in C^{\infty}\left(U \times\left(\mathbb{R}^{n} \backslash\{0\}\right)\right), p_{(\mu-j)}(x, \lambda \xi)=\lambda^{\mu-j} p_{(\mu-j)}(x, \xi)$ for all $\lambda \in \mathbb{R}_{+}$, and $\chi$ is any excision function. If some assertion is valid for the classical and the general case we also write $S_{(\mathrm{cl})}^{\mu}\left(U \times \mathbb{R}^{n}\right)$. Recall that the spaces $S_{(\mathrm{cl})}^{\mu}\left(U \times \mathbb{R}^{n}\right)$ are Fréchet in a natural way. It is then obvious that $S_{(\mathrm{cl})}^{\mu}\left(\mathbb{R}^{n}\right)$ (the space of $x$-independent elements) is closed in $S_{(\mathrm{cl})}^{\mu}\left(U \times \mathbb{R}^{n}\right)$, and that

$$
S_{(\mathrm{cl})}^{\mu}\left(U \times \mathbb{R}^{n}\right)=C^{\infty}\left(U, S_{(\mathrm{cl})}^{\mu}\left(\mathbb{R}^{n}\right)\right) .
$$

In order to illustrate some consequences of the presence of a boundary, here $t=0$, we rephrase (4.1) in anisotropic form, by carrying out the action first in $t$ and then in $y$. It will be not essential that $y$ varies in $\mathbb{R}^{n-1}$; we often assume $y \in \Omega$ for an open set $\Omega \subseteq \mathbb{R}^{n-1}$. Moreover, for simplicity, we first consider a $t$-independent symbol, i.e., $p(y, \eta, \tau) \in S^{\mu}\left(\Omega \times \mathbb{R}_{\eta}^{n-1} \times \mathbb{R}_{\tau}\right)$. We form $\mathrm{Op}_{t}(p)(y, \eta)$ : $H^{s}(\mathbb{R}) \rightarrow H^{s-\mu}(\mathbb{R})$ as an operator family parametrised by $(y, \eta) \in \Omega \times \mathbb{R}^{n-1}$ and then

$$
\mathrm{Op}_{x}(p)=\mathrm{Op}_{y}\left(\mathrm{Op}_{t}(p)\right)
$$

where $\mathrm{Op}_{t}(p)(y, \eta)$ is regarded as an operator-valued symbol in the variables and covariables $(y, \eta)$.

In order to formulate the latter aspect in a more precise manner we fix a group $\left\{\kappa_{\lambda}\right\}_{\lambda \in \mathbb{R}_{+}}$of isomorphisms $\kappa_{\lambda}: H^{s}(\mathbb{R}) \rightarrow H^{s}(\mathbb{R})$ by setting $\left(\kappa_{\lambda} u\right)(t)=\lambda^{1 / 2} u(\lambda t)$, $\lambda \in \mathbb{R}_{+}$. Then a simple computation shows the identity

$$
\kappa_{\langle\eta\rangle}^{-1} \mathrm{Op}_{t}(p)(y, \eta) \kappa_{\langle\eta\rangle}=\mathrm{Op}_{t}\left(p_{\eta}\right)(y, \eta)
$$

for

$$
p_{\eta}(y, \eta, \tau)=p(y, \eta\langle\tau\rangle, \tau) .
$$

Using the symbolic estimates for $p$, especially, $|p(y, \eta, \tau)| \leq c\langle\eta, \tau\rangle^{\mu}$ for all $(y, \eta, \tau) \in K \times \mathbb{R}^{n}, K \Subset \Omega$, and constants $c(K)>0$, it follows that

$$
|p(y, \eta\langle\tau\rangle, \tau)| \leq c\langle\eta\rangle^{\mu}\langle\tau\rangle^{\mu},
$$

taking into account the relation $\langle\eta\langle\tau\rangle, \tau\rangle=\langle\eta\rangle\langle\tau\rangle$.

Lemma 4.1. Under the above assumptions we have

$$
\left\|\kappa_{\langle\eta\rangle}^{-1}\left\{D_{y}^{\alpha} D_{\eta}^{\beta} \mathrm{Op}_{t}(p)(y, \eta)\right\} \kappa_{\langle\eta\rangle}\right\|_{\mathcal{L}\left(H^{s}(\mathbb{R}), H^{s-\mu+|\beta|}(\mathbb{R})\right)} \leq c\langle\eta\rangle^{\mu-|\beta|}
$$

for all $(y, \eta) \in K \times \mathbb{R}^{n-1}, K \Subset \Omega$, and all $\alpha, \beta \in \mathbb{N}^{n-1}$, and every $s \in \mathbb{R}$, for constants $c=c(\alpha, \beta, K, s)>0$.

Proof. Let first $\alpha=\beta=0$, and set $a(y, \eta):=\mathrm{Op}_{t}(p)(y, \eta)$. Then the relation (4.3) together with the estimate (4.5) yields

$$
\begin{aligned}
& \left\|\kappa_{\langle\eta\rangle}^{-1} a(y, \eta) \kappa_{\langle\eta\rangle} u\right\|_{H^{s-\mu}(\mathbb{R})}^{2}=\int\langle\tau\rangle^{2(s-\mu)}|p(y, \eta\langle\tau\rangle, \tau) \hat{u}(\tau)|^{2} d \tau \\
& \quad \leq \sup _{\tau \in \mathbb{R}, y \in K}\langle\tau\rangle^{-2 \mu}|p(y, \eta\langle\tau\rangle, \tau)|^{2} \int\langle\tau\rangle^{2 s}|\hat{u}(\tau)|^{2} d \tau \leq c\langle\eta\rangle^{2 \mu}\|u\|_{H^{s}(\mathbb{R})}^{2}
\end{aligned}
$$


This implies (4.6) for $\alpha=\beta=0$. The assertion for arbitrary $\alpha, \beta$ follows in an analogous manner, using $D_{y}^{\alpha} D_{\eta}^{\beta} p(y, \eta, \tau) \in S^{\mu-|\beta|}\left(U \times \mathbb{R}^{n}\right)$.

Remark 4.2. Lemma 4.1 remains true in analogous form under the assumption $p(y, t, \eta, \tau) \in S^{\mu}\left(\Omega \times \mathbb{R} \times \mathbb{R}_{\eta, \tau}^{n}\right)$ when $p$ is independent of $t$ for $|t|>$ const for a constant $>0$ (and also under certain weaker assumptions with respect to $|t| \rightarrow \infty$ ).

Definition 4.3. $\quad$ 1. By a group action on a Hilbert space $H$ we understand a strongly continuous group $\kappa=\left\{\kappa_{\lambda}\right\}_{\lambda \in \mathbb{R}_{+}}$of isomorphisms $\kappa_{\lambda}: H \rightarrow H$, such that $\kappa_{\lambda \lambda^{\prime}}=\kappa_{\lambda} \kappa_{\lambda^{\prime}}$ for all $\lambda, \lambda^{\prime} \in \mathbb{R}_{+}$(strongly continuous means that $\kappa_{\lambda} h \in C\left(\mathbb{R}_{+}, H\right)$ for every $\left.h \in H\right)$.

2. Let $H$ and $\widetilde{H}$ be Hilbert spaces with group actions $\kappa$ and $\tilde{\kappa}$, respectively. Then $S^{\mu}\left(\Omega \times \mathbb{R}^{q} ; H, \widetilde{H}\right)$ for $\Omega \subseteq \mathbb{R}^{p}$ open, $\mu \in \mathbb{R}$, is defined to be the set of all $a(y, \eta) \in C^{\infty}\left(\Omega \times \mathbb{R}^{q}, \mathcal{L}(\bar{H}, \widetilde{H})\right)$ such that

$$
\left\|\tilde{\kappa}_{\langle\eta\rangle}^{-1}\left\{D_{y}^{\alpha} D_{\eta}^{\beta} a(y, \eta)\right\} \kappa_{\langle\eta\rangle}\right\|_{\mathcal{L}(H, \widetilde{H})} \leq c\langle\eta\rangle^{\mu-|\beta|}
$$

for all $(y, \eta) \in K \times \mathbb{R}^{q}, K \Subset \Omega$, and all $\alpha \in \mathbb{N}^{p}, \beta \in \mathbb{N}^{q}$, for constants $c=c(\alpha, \beta, K)>0$.

3. The space $S_{\mathrm{cl}}^{\mu}\left(\Omega \times \mathbb{R}^{q} ; H, \widetilde{H}\right)$ of classical elements is the set of all $a(y, \eta) \in$ $S^{\mu}\left(\Omega \times \mathbb{R}^{q} ; H, \widetilde{H}\right)$ such that there are functions $a_{(\mu-j)}(y, \eta) \in C^{\infty}(\Omega \times$ $\left.\left(\mathbb{R}^{q} \backslash\{0\}\right), \mathcal{L}(H, \widetilde{H})\right), j \in \mathbb{N}$, with $a_{(\mu-j)}(y, \lambda \eta)=\lambda^{\mu-j} \tilde{\kappa}_{\lambda} a_{(\mu-j)}(y, \eta) \kappa_{\lambda}^{-1}$ for all $\lambda \in \mathbb{R}_{+},(y, \eta) \in \Omega \times\left(\mathbb{R}^{q} \backslash\{0\}\right)$, with

$$
a(y, \eta)-\sum_{j=0}^{N} \chi(\eta) a_{(\mu-j)}(y, \eta) \in S^{\mu-(N+1)}\left(\Omega \times \mathbb{R}^{q} ; H, \widetilde{H}\right)
$$

for every $N \in \mathbb{N}$ and any excision function $\chi(\eta)$.

Example. $\quad$ 1. For $p(y, \eta, \tau) \in S^{\mu}\left(\Omega \times \mathbb{R}^{n}\right)$ and $a(y, \eta)=\mathrm{Op}_{t}(p)(y, \eta)$ we have $a(y, \eta) \in S_{\mathrm{cl}}^{\mu}\left(\Omega \times \mathbb{R}^{n-1} ; H^{s}(\mathbb{R}), H^{s-\mu}(\mathbb{R})\right)$ for every $s \in \mathbb{R}$.

2. For $p(y, t, \eta, \tau) \in S^{\mu}\left(\Omega \times \mathbb{R} \times \mathbb{R}^{n}\right)$ under the assumption of Remark 4.2 we have $a(y, \eta) \in S^{\mu}\left(\Omega \times \mathbb{R}^{n-1} ; H^{s}(\mathbb{R}), H^{s-\mu}(\mathbb{R})\right)$ for every $s \in \mathbb{R}$.

Remark 4.4. Observe that in the latter Example we did not exhaust the full information of (4.6) with respect to $s$. In fact, differentiation in $\eta$ gives us better smoothness in the image spaces. For our purposes it suffices to fix the Hilbert spaces $H$ and $\widetilde{H}$; in applications it will be clear anyway to what extent we can say more when those spaces run over scales of spaces, parametrised by $s$.

Parallel to the spaces of operator-valued symbols we have vector-valued analogues of Sobolev spaces.

Definition 4.5. Let $H$ be a Hilbert space with group action $\kappa=\left\{\kappa_{\lambda}\right\}_{\lambda \in \mathbb{R}_{+}}$. Then $\mathcal{W}^{s}\left(\mathbb{R}^{q}, H\right)$ for $s \in \mathbb{R}$ is defined to be the completion of $\mathcal{S}\left(\mathbb{R}^{q}, H\right)$ with respect to the norm $\left\|\langle\eta\rangle^{s} \kappa_{\langle\eta\rangle}^{-1} \hat{u}(\eta)\right\|_{L^{2}\left(\mathbb{R}^{q}, H\right)}$. 
The space $\mathcal{W}^{s}\left(\mathbb{R}^{q}, H\right)$ is contained in $\mathcal{S}^{\prime}\left(\mathbb{R}^{q}, H\right)=\mathcal{L}\left(\mathcal{S}\left(\mathbb{R}^{q}\right), H\right)$. For every open $\Omega \subseteq \mathbb{R}^{q}$ we define $\mathcal{W}_{\text {comp }}^{s}(\Omega, H)$ to be the set of all $u \in \mathcal{W}^{s}\left(\mathbb{R}^{q}, H\right)$ with compact support and $\mathcal{W}_{\text {loc }}^{s}(\Omega, H) \subset \mathcal{D}^{\prime}(\Omega, H)=\mathcal{L}\left(C_{0}^{\infty}(\Omega), H\right)$ by $\varphi u \in \mathcal{W}_{\text {comp }}^{s}(\Omega, H)$ for every $\varphi \in C_{0}^{\infty}(\Omega)$.

Example. $\quad 1$. Let $H:=H^{s}\left(\mathbb{R}^{m}\right),\left(\kappa_{\lambda} u\right)(x):=\lambda^{m / 2} u(\lambda x)$ for $\lambda \in \mathbb{R}_{+}$. Then for every $s \in \mathbb{R}$ we have

$$
\mathcal{W}^{s}\left(\mathbb{R}^{q}, H^{s}\left(\mathbb{R}^{m}\right)\right)=H^{s}\left(\mathbb{R}^{q} \times \mathbb{R}^{m}\right) .
$$

2. Let $H:=H^{s}\left(\mathbb{R}_{+}\right),\left(\kappa_{\lambda} u\right)(t)=\lambda^{1 / 2} u(\lambda t), \lambda \in \mathbb{R}_{+}$. Then for every $s \in \mathbb{R}$ we have

$$
\mathcal{W}^{s}\left(\mathbb{R}^{q}, H^{s}\left(\mathbb{R}_{+}\right)\right)=H^{s}\left(\mathbb{R}^{q} \times \mathbb{R}_{+}\right) .
$$

Remark 4.6. The notion of group actions also makes sense for Fréchet spaces that are written as projective limits of Hilbert spaces. An example is the Schwartz space

$$
\mathcal{S}\left(\mathbb{R}^{m}\right)=\lim _{j \in \mathbb{N}}\langle x\rangle^{-j} H^{j}\left(\mathbb{R}^{m}\right)
$$

with $\kappa_{\lambda}$ being defined as in the above Example. There are then natural extensions of Definitions 4.3 and 4.5 as well as comp/loc spaces to the case of Fréchet spaces with group action (for more details cf. also [17], [18]).

Theorem 4.7. Let $H$ and $\widetilde{H}$ be Hilbert (Fréchet) spaces with group action and $a(y, \eta) \in S^{\mu}\left(\Omega \times \mathbb{R}^{q} ; H, \widetilde{H}\right)$. Then $\mathrm{Op}_{y}(a): C_{0}^{\infty}(\Omega, H) \rightarrow C^{\infty}(\Omega, \widetilde{H})$ extends to a continuous operator

$$
\mathrm{Op}_{y}(a): \mathcal{W}_{\mathrm{comp}}^{s}(\Omega, H) \rightarrow \mathcal{W}_{\mathrm{loc}}^{s-\mu}(\Omega, \widetilde{H})
$$

for every $s \in \mathbb{R}$. If $a(y, \eta) \in S^{\mu}\left(\mathbb{R}^{q} \times \mathbb{R}^{q} ; H, \widetilde{H}\right)$ is independent of $y$ for $|y| \geq$ const $>0$ then we obtain a continuous operator

$$
\mathrm{Op}_{y}(a): \mathcal{W}^{s}\left(\mathbb{R}^{q}, H\right) \rightarrow \mathcal{W}^{s-\mu}\left(\mathbb{R}^{q}, \widetilde{H}\right)
$$

for every $s \in \mathbb{R}$.

Remark 4.8. The continuity of (4.8) can be proved under much more general assumptions on $a(y, \eta)$ than in Theorem 4.7, see, for instance, [17] or [28].

Let us now turn to what we did at the beginning of this section. For $p(y, t, \eta, \tau) \in$ $S^{\mu}\left(\mathbb{R}^{n-1} \times \mathbb{R} \times \mathbb{R}^{n}\right)$ we have

$$
\mathrm{Op}_{t}(p)(y, \eta) \in S^{\mu}\left(\mathbb{R}^{n-1} \times \mathbb{R}^{n-1} ; H^{s}(\mathbb{R}), H^{s-\mu}(\mathbb{R})\right)
$$

when $p$ satisfies the assumption of Remark 4.2. For our purposes it suffices to assume that $p$ is a classical symbol of order $\mu \in \mathbb{Z}$, and independent of $(y, t)$ for $|y, t| \geq$ const for some constant $>0$.

In a theory of elliptic boundary value problems that relies on standard Sobolev spaces $H^{s}\left(\mathbb{R}_{+}^{n}\right)=\left.H^{s}\left(\mathbb{R}^{n}\right)\right|_{\mathbb{R}_{+}^{n}}$ we should possess the continuity of

$$
\mathrm{Op}^{+}(p)=\mathrm{r}^{+} \mathrm{Op}(p) \mathrm{e}^{+}: H^{s}\left(\mathbb{R}_{+}^{n}\right) \rightarrow H^{s-\mu}\left(\mathbb{R}_{+}^{n}\right)
$$


for $s>-1 / 2$, similarly as in Corollary 3.9 ; here $\mathrm{e}^{+}$is the operator of extension by zero from $\mathbb{R}_{+}^{n}$ to $\mathbb{R}^{n}$, and $\mathrm{r}^{+}$the restriction to $\mathbb{R}_{+}^{n}$ (analogously we have the extension and restriction operators $\mathrm{e}^{-}$and $\mathrm{r}^{-}$, respectively). It turns out that the continuity of (4.9) requires certain very restrictive assumptions on the symbol $p$. For instance, for $p(x, \xi)=\chi(\xi)|\xi|$ where $\chi$ is some excision function the operator (4.9) will not be continuous for all $s>-\frac{1}{2}$.

According to Theorem 4.7 for the continuity in Sobolev spaces it suffices to know that

$$
\mathrm{op}^{+}(p)(y, \eta) \in S^{\mu}\left(\mathbb{R}^{n-1} \times \mathbb{R}^{n-1} ; H^{s}\left(\mathbb{R}_{+}\right), H^{s-\mu}\left(\mathbb{R}_{+}\right)\right)
$$

for $s>-\frac{1}{2}$, i.e.,

$$
\left\|\kappa_{\langle\eta\rangle}^{-1}\left\{D_{y}^{\alpha} D_{\eta}^{\beta} \mathrm{op}^{+}(p)(y, \eta)\right\} \kappa_{\langle\eta\rangle}\right\|_{\mathcal{L}\left(H^{s}\left(\mathbb{R}_{+}\right), H^{s-\mu}\left(\mathbb{R}_{+}\right)\right)} \leq c\langle\eta\rangle^{\mu-|\beta|}
$$

for all $(y, \eta) \in \mathbb{R}^{n-1} \times \mathbb{R}^{n-1}$, and all $\alpha, \beta$, for $c=c(\alpha, \beta, K, s)>0$.

Moreover, it is desirable to have

$$
\operatorname{op}^{+}(p)(y, \eta) \in S^{\mu}\left(\mathbb{R}^{n-1} \times \mathbb{R}^{n-1} ; \mathcal{S}\left(\overline{\mathbb{R}}_{+}\right), \mathcal{S}\left(\overline{\mathbb{R}}_{+}\right)\right) .
$$

In order to illustrate the effect for the moment we consider the case that that $p$ is independent of $y$ and $t$. To obtain (4.11) we assume

$$
\tilde{p}(\eta, \tau):=p(\eta\langle\tau\rangle, \tau) \in S^{\mu}\left(\mathbb{R}^{q}, S_{\mathrm{tr}}^{\mu}(\mathbb{R})\right) .
$$

The notation $S^{\mu}\left(\mathbb{R}^{q}, E\right)$ for a Fréchet space $E$ with the semi-norm system $\left(\pi_{k}\right)_{k \in \mathbb{N}}$ means the set of all $a(\eta) \in C^{\infty}\left(\mathbb{R}^{q}, E\right)$ such that

$$
\pi_{k}\left(D_{\eta}^{\beta} a(\eta)\right) \leq c\langle\eta\rangle^{\mu-|\beta|}
$$

for all $\eta \in \mathbb{R}^{q}, \beta \in \mathbb{N}^{q}, k \in \mathbb{N}$, for constants $c=c(\beta, k)>0$.

Lemma 4.9. Let $E$ and $F$ be Fréchet spaces with the semi-norm systems $\left(\pi_{j}\right)_{j \in \mathbb{N}}$ and $\left(\sigma_{j}\right)_{j \in \mathbb{N}}$, respectively, and let $B: E \rightarrow F$ be a continuous operator. Then

$$
T_{B}: C^{\infty}\left(\mathbb{R}^{q}, E\right) \rightarrow C^{\infty}\left(\mathbb{R}^{q}, F\right)
$$

defined by the composition $a: \mathbb{R}^{q} \rightarrow E$ and $B: E \rightarrow F$ induces a continuous operator

$$
T_{B}: S^{\mu}\left(\mathbb{R}^{q}, E\right) \rightarrow S^{\mu}\left(\mathbb{R}^{q}, F\right)
$$

for every $\mu \in \mathbb{R}$.

Proof. Without loss of generality we assume $\sigma_{j+1}(\cdot) \geq \sigma_{j}(\cdot)$ and $\pi_{j+1}(\cdot) \geq \pi_{j}(\cdot)$ for all $j$. Then continuity of $B$ means that for every $k \in \mathbb{N}$ there is a $j \in \mathbb{N}$ such that $\sigma_{k}(B u) \leq c \pi_{j}(u)$ for all $u \in E$, for some $c>0$. Analogously, the continuity of (4.15) means that for every $k \in \mathbb{N}, \beta \in \mathbb{N}^{q}$, there are $j, N \in \mathbb{N}$ such that

$$
\sup _{\eta \in \mathbb{R}^{q}}\langle\eta\rangle^{\mu+|\beta|} \sigma_{k}\left(D_{\eta}^{\beta} T_{B} a(\eta)\right) \leq c \sup _{\substack{\eta \in \mathbb{R}^{q} \\|\alpha| \leq N}}\langle\eta\rangle^{-\mu+|\alpha|} \pi_{j}\left(D_{\eta}^{\alpha} a(\eta)\right)
$$


for some $c>0$. Since $T_{B} a(\eta)=(B a)(\eta)$ with pointwise composition and $D_{\eta}^{\alpha}(B a)(\eta)=$ $B\left(D_{\eta}^{\alpha} a\right)(\eta)$ it follows that

$$
\sup _{\eta \in \mathbb{R}^{q}}\langle\eta\rangle^{-\mu+|\beta|} \sigma_{k}\left(D_{\eta}^{\beta} T_{B} a(\eta)\right) \leq c \sup _{\eta \in \mathbb{R}^{q}}\langle\eta\rangle^{-\mu+|\beta|} \pi_{j}\left(D_{\eta}^{\alpha} a(\eta)\right)
$$

which implies (4.16).

Lemma 4.10. Let $p(y, \eta, \tau) \in C^{\infty}\left(\mathbb{R}^{n-1}, S_{\mathrm{cl}}^{\mu}\left(\mathbb{R}^{n}\right)\right)$ and

$$
\tilde{p}(y, \eta, \tau) \in C^{\infty}\left(\mathbb{R}^{n-1}, S^{\mu}\left(\mathbb{R}^{n-1}, S_{\mathrm{tr}}^{\mu}(\mathbb{R})\right)\right) .
$$

Then we have the relations (4.10) for $s>-\frac{1}{2}$, and (4.12).

Proof. For (4.10) we have to verify the estimates (4.11). Let first $\alpha=\beta=0$. For simplicity let $p$ be independent of $y$. An analogue of the relations (4.4) gives us

$$
\kappa_{\langle\eta\rangle}^{-1} \mathrm{op}^{+}(p)(\eta) \kappa_{\langle\eta\rangle}=\mathrm{op}^{+}(\tilde{p})(\eta) .
$$

The operation $\mathrm{op}^{+}(\cdot)$ induces a continuous operator

$$
\mathrm{op}^{+}(\cdot): S_{\mathrm{tr}}^{\mu}(\mathbb{R}) \rightarrow \mathcal{L}\left(H^{s}\left(\mathbb{R}_{+}\right), H^{s-\mu}\left(\mathbb{R}_{+}\right)\right)
$$

for every $s>-\frac{1}{2}$. That means for every $s$ there is a semi-norm $\pi_{j}$ from the Fréchet topology of $S_{\mathrm{tr}}^{\mu}(\mathbb{R})$ such that

$$
\left\|\mathrm{op}^{+}(a)\right\|_{\mathcal{L}\left(H^{s}\left(\mathbb{R}_{+}\right), H^{s-\mu}\left(\mathbb{R}_{+}\right)\right)} \leq c \pi_{j}(a)
$$

for every $a \in S_{\mathrm{tr}}^{\mu}(\mathbb{R})$. Thus, for $E=S_{\mathrm{tr}}^{\mu}(\mathbb{R}), \widetilde{F}=\mathcal{L}\left(H^{s}\left(\mathbb{R}_{+}\right), H^{s-\mu}\left(\mathbb{R}_{+}\right)\right)$from Lemma 4.9 it follows that

$$
\sup \langle\eta\rangle^{-\mu}\left\|\operatorname{op}^{+}(\tilde{p})(\eta)\right\|_{\mathcal{L}\left(H^{s}\left(\mathbb{R}_{+}\right), H^{s-\mu}\left(\mathbb{R}_{+}\right)\right)} \leq c \sup \langle\eta\rangle^{-\mu} \pi_{j}(\tilde{p}(\eta, \cdot))<\infty,
$$

i.e., using (4.17) that

$$
\left\|\kappa_{\langle\eta\rangle}^{-1} \mathrm{op}^{+}(p)(\eta) \kappa_{\langle\eta\rangle}\right\|_{\mathcal{L}\left(H^{s}\left(\mathbb{R}_{+}\right), H^{s-\mu}\left(\mathbb{R}_{+}\right)\right)} \leq c\langle\eta\rangle^{\mu}
$$

In a similar manner we can proceed with the derivatives $D_{\eta}^{\beta} p(\eta, \tau)$ for every $\beta \in$ $\mathbb{N}^{n-1}$.

The proof (4.12) is straightforward as well and left to the reader.

Definition 4.11. A symbol $p(y, t, \eta, \tau) \in S_{\mathrm{cl}}^{\mu}\left(\Omega_{y} \times \mathbb{R} \times \mathbb{R}_{\eta, \tau}^{n}\right)$ for $\mu \in \mathbb{Z}$ is said to have the transmission property at $t=0$ if the homogeneous components $p_{(\mu-j)}$ of $p$ satisfy the conditions

$$
D_{y, t}^{\alpha} D_{\eta, \tau}^{\beta}\left\{p_{(\mu-j)}(y, t, \eta, \tau)-(-1)^{\mu-j} p_{(\mu-j)}(y, t,-\eta,-\tau)\right\}=0
$$

on the set $\left\{(y, t, \eta, \tau) \in \Omega \times \mathbb{R} \times \mathbb{R}^{n}: y \in \Omega, t=0, \eta=0, \tau \in \mathbb{R} \backslash\{0\}\right\}$ of non-vanishing conormal vectors over the boundary, for all $\alpha, \beta \in \mathbb{N}^{n}, j \in \mathbb{N}$. Let $S_{\mathrm{tr}}^{\mu}\left(\Omega \times \mathbb{R} \times \mathbb{R}^{n}\right)$ denote the space of all symbols of that kind. Moreover, set

$$
S_{\mathrm{tr}}^{\mu}\left(\Omega \times \overline{\mathbb{R}}_{ \pm} \times \mathbb{R}^{n}\right):=\left\{\left.p\right|_{\Omega \times \overline{\mathbb{R}}_{ \pm} \times \mathbb{R}^{n}}: p \in S_{\mathrm{tr}}^{\mu}\left(\Omega \times \mathbb{R} \times \mathbb{R}^{n}\right)\right\}
$$


Since the transmission property is a local condition near $t=0$ it can easily be extended to symbols in an arbitrary open set $U \subseteq \mathbb{R}^{n}$ intersecting $\{t=$ $0\}$. (It is clear that it suffices to ask (4.18) only for all $\alpha=\left(0, \ldots, \alpha_{n}\right), \beta=$ $\left.\left(\beta_{1}, \ldots, \beta_{n-1}, 0\right)\right)$.

Operators with symbols with the transmission property in connection with boundary value problems (and also transmission problems) have been studied by many outhors, first of all Boutet de Monvel [5], [4], Eskin [7], and later on Myshkis [14], Rempel and Schulze [15], Grubb [8], [9], and many others. One of the main motivations was to find a framework to express parametrices of elliptic boundary value problems for differential operators and to prove an analogue of the Atiyah-Singer index theorem. In this connection it appeared not too perturbing that generically symbols (that are smooth up to the boundary) have not the transmission property at the boundary. We will return to more general symbols below.

The first important aspect is that a pseudo-differential theory of boundary value problems concerns continuous operators (4.9) (and analogously on manifolds with smooth boundary). Another essential point is to understand the behaviour of such operators under compositions.

Proposition 4.12. For every $p(y, t, \eta, \tau) \in S_{\mathrm{tr}}^{\mu}\left(\Omega \times \overline{\mathbb{R}}_{+} \times \mathbb{R}^{n}\right)$ we have

$$
\tilde{p}(y, t, \eta, \tau):=p(y, t, \eta\langle\tau\rangle, \tau) \in C^{\infty}\left(\Omega \times \overline{\mathbb{R}}_{+}, S^{\mu}\left(\mathbb{R}^{n-1}, S_{\mathrm{tr}}^{\mu}(\mathbb{R})\right)\right) .
$$

The simple proof is left to the reader.

In the local analysis of BVPs it suffices to assume that the involved symbols are independent of $t$ for large $t$.

Proposition 4.13. For every $p(y, t, \eta, \tau) \in S_{\mathrm{tr}}^{\mu}\left(\Omega \times \overline{\mathbb{R}}_{+} \times \mathbb{R}^{n}\right)$ which is independent of $t$ for large $t$ we have

$$
\mathrm{op}^{+}(p)(y, \eta) \in S^{\mu}\left(\Omega \times \mathbb{R}^{q} ; H^{s}\left(\mathbb{R}_{+}\right), H^{s-\mu}\left(\mathbb{R}_{+}\right)\right)
$$

for every $s>-\frac{1}{2}$, and

$$
\mathrm{op}^{+}(p)(y, \eta) \in S^{\mu}\left(\Omega \times \mathbb{R}^{q} ; \mathcal{S}\left(\overline{\mathbb{R}}_{+}\right), \mathcal{S}\left(\overline{\mathbb{R}}_{+}\right)\right) .
$$

The $t$-independent case is contained in Lemma 4.10. After that the proof in general is straightforward.

Theorem 4.7 together with (4.19) entails the continuity of

$$
\mathrm{Op}^{+}(p)=\mathrm{Op}_{y}\left(\mathrm{op}^{+}(p)\right): H_{[\mathrm{comp})}^{s}\left(\Omega \times \mathbb{R}_{+}\right) \rightarrow H_{[\mathrm{loc})}^{s-\mu}\left(\Omega \times \mathbb{R}_{+}\right) ;
$$

here $H_{\text {[comp } /[\text { loc })}^{s}\left(\Omega \times \mathbb{R}_{+}\right)=\mathcal{W}_{\text {comp } / \text { loc }}^{s}\left(\Omega, H^{s}\left(\mathbb{R}_{+}\right)\right)$, cf. also Example 4 (ii). Let us now give a motivation of the conditions (4.18) in Definition 4.11. First it is evident that when $p$ is a polynomial in $\xi$, the homogeneous components $p_{(\mu-j)}$ of order $\mu-j, j=0, \ldots, \mu$, satisfy the relations (4.18). For instance, we have in this case

$$
p_{(\mu)}(y, t, \lambda \eta, \lambda \tau)=\lambda^{\mu} p_{(\mu)}(y, t, \eta, \tau)
$$

for every $\lambda \in \mathbb{R}$, not only for $\lambda \in \mathbb{R}_{+}$, and hence,

$$
p_{(\mu)}(y, t, \eta, \tau)=(-1)^{\mu} p_{(\mu)}(y, t,-\eta,-\tau),
$$


even for all $(y, t, \eta, \tau)$.

If $p(x, \xi)$ is elliptic of order $\mu$ then the Leibniz inverse which belongs to $S_{\mathrm{cl}}^{-\mu}\left(\Omega \times \mathbb{R} \times \mathbb{R}_{\xi}^{n}\right)$ satisfies those conditions as well with respect to the order $-\mu$.

The behaviour of operators under compositions locally near the boundary can be reduced to the composition of operators with operator-valued symbols, modulo smoothing operators. In general, if $H, \widetilde{H}$, and $\widetilde{\widetilde{H}}$ are Hilbert spaces with group actions $\kappa=\left\{\kappa_{\lambda}\right\}_{\lambda \in \mathbb{R}_{+}}, \tilde{\kappa}=\left\{\tilde{\kappa}_{\lambda}\right\}_{\lambda \in \mathbb{R}_{+}}$, and $\tilde{\tilde{\kappa}}=\left\{\tilde{\tilde{\kappa}}_{\lambda}\right\}_{\lambda \in \mathbb{R}_{+}}$, respectively, and

$$
a(y, \eta) \in S^{\mu}\left(\Omega \times \mathbb{R}^{q}, \widetilde{H}, \widetilde{\widetilde{H}}\right), \tilde{a}(y, \eta) \in S^{\tilde{\mu}}\left(\Omega \times \mathbb{R}^{q} ; H, \widetilde{H}\right),
$$

for simplicity, with compact support with respect to $y$, then we can form

$$
\mathrm{Op}_{y}(a) \mathrm{Op}_{y}(\tilde{a})=\mathrm{Op}(a \# \tilde{a})
$$

with the Leibniz product $a \# \tilde{a}(y, \eta) \in S^{\mu+\tilde{\mu}}\left(\Omega \times \mathbb{R}^{q} ; H, \widetilde{\widetilde{H}}\right)$ that can be computed by an operator-valued analogue of the respective oscillatory integral expression in Kumano-go's formalism. This entails an asymptotic expansion

$$
a \# \tilde{a}(y, \eta) \sim \sum_{\alpha \in \mathbb{N}^{q}} \frac{1}{\alpha !}\left(\partial_{\eta}^{\alpha} a(y, \eta)\right) D_{y}^{\alpha} \tilde{a}(y, \eta),
$$

$\partial_{\eta}^{\alpha}:=\partial^{\alpha_{1}} / \partial y_{1}^{\alpha_{1}} \ldots \partial^{\alpha_{q}} / \partial y_{q}^{\alpha_{q}}$

If we apply this to the case

$$
a(y, \eta)=\mathrm{op}^{+}(p)(y, \eta), \tilde{a}(y, \eta)=\mathrm{op}^{+}(\tilde{p})(y, \eta)
$$

for symbols $p(x, \xi) \in S_{\mathrm{tr}}^{\mu}\left(\Omega \times \mathbb{R} \times \mathbb{R}^{n}\right), \tilde{p}(x, \xi) \in S_{\mathrm{tr}}^{\tilde{\mu}}\left(\Omega \times \mathbb{R} \times \mathbb{R}^{n}\right)$, (say, under the simplifying condition of compact support in $(y, t))$ then we have to understand the compositions

$$
\left(\partial_{\eta}^{\alpha} \mathrm{op}^{+}(p)(y, \eta)\right) D_{y}^{\alpha} \mathrm{op}^{+}(\tilde{p})(y, \eta)=\mathrm{op}^{+}\left(\partial_{\eta}^{\alpha} p\right)(y, \eta) \mathrm{op}^{+}\left(D_{y}^{\alpha} \tilde{p}\right)(y, \eta)
$$

Since $\mu, \tilde{\mu} \in \mathbb{Z}$ are arbitrary, and $\partial_{\eta}^{\alpha} p \in S_{\mathrm{tr}}^{\mu-|\alpha|}, D_{y}^{\alpha} \tilde{p} \in S_{\mathrm{tr}}^{\tilde{\mu}}$, we may consider, for instance, the case $\alpha=0$. From the information of Section 3 we know that

$$
\mathrm{op}^{+}(p)(y, \eta) \mathrm{op}^{+}(\tilde{p})(y, \eta)=\mathrm{op}^{+}\left(p \#{ }_{t} \tilde{p}\right)(y, \eta)+g(y, \eta)
$$

where $p \#_{t} \tilde{p}$ is the Leibniz product between $p$ and $\tilde{p}$ with respect to the $t$-variable, and $g(y, \eta)$ is a family of operators in $\Gamma^{0}\left(\mathbb{R}_{+}\right)$.

More precisely, the operator families $g(y, \eta)$ are Green symbols in the following sense.

Definition 4.14. 1 . An operator-valued symbol $g(y, \eta)$ belongs to $\mathcal{R}_{G}^{\mu, 0}(\Omega \times$ $\left.\mathbb{R}^{n-1}\right)$ if

$$
g(y, \eta), g^{*}(y, \eta) \in S_{\mathrm{cl}}^{\mu}\left(\Omega \times \mathbb{R}^{n-1} ; L^{2}\left(\mathbb{R}_{+}\right), \mathcal{S}\left(\overline{\mathbb{R}}_{+}\right)\right) .
$$

Here $g^{*}(y, \eta)$ is the $(y, \eta)$-wise $L^{2}\left(\mathbb{R}_{+}\right)$-adjoint. Elements of $\mathcal{R}_{G}^{\mu, 0}\left(\Omega \times \mathbb{R}^{n-1}\right)$ are called Green symbols of type 0 . 
2. An operator family $g(y, \eta)$ belongs to $\mathcal{R}_{G}^{\mu, d}\left(\Omega \times \mathbb{R}^{n-1}\right)$, then called a Green symbol of type $d \in \mathbb{N}$, if

$$
g(y, \eta)=\sum_{j=0}^{d} g_{j}(y, \eta) \partial_{t}^{j}
$$

for $g_{j}(y, \eta) \in \mathcal{R}_{G}^{\mu-j, 0}\left(\Omega \times \mathbb{R}^{n-1}\right), j=0, \ldots, d$.

Similarly as (3.23) we also define $2 \times 2$ block matrices

$$
\boldsymbol{a}(y, \eta):=\left(\begin{array}{cc}
\mathrm{op}^{+}(p)(y, \eta)+g_{11}(y, \eta) & g_{12}(y, \eta) \\
g_{21}(y, \eta) & g_{22}(y, \eta)
\end{array}\right): \begin{gathered}
H^{s}\left(\mathbb{R}_{+}\right) \\
\mathbb{C}^{j_{-}}
\end{gathered} \rightarrow \begin{gathered}
H^{s-\mu}\left(\mathbb{R}_{+}\right) \\
\mathbb{C}^{j_{+}}
\end{gathered}
$$

for arbitrary $p(y, t, \eta, \tau) \in S_{\mathrm{tr}}^{\mu}\left(\Omega \times \mathbb{R} \times \mathbb{R}_{\eta, \tau}^{n}\right)$ (independent of $t$ for $|t|>$ const), and $g_{11}(y, \eta) \in \mathcal{R}_{G}^{\mu, d}\left(\Omega \times \mathbb{R}^{q}\right), s>d-\frac{1}{2}$, while (say, for the case $j_{-}=j_{+}=1$ )

$$
g_{12}(y, \eta) \in S_{\mathrm{cl}}^{\mu}\left(\Omega \times \mathbb{R}^{n-1} ; \mathbb{C}, \mathcal{S}\left(\overline{\mathbb{R}}_{+}\right)\right)
$$

(with $\mathbb{C}$ being endowed with the trivial group action),

$$
g_{21}(y, \eta) u(t)=\sum_{l=0}^{d} g_{21, l}(y, \eta) \partial_{t}^{l} u(t)
$$

for $g_{21, l}^{*}(y, \eta) \in S_{\mathrm{cl}}^{\mu-l}\left(\Omega \times \mathbb{R}^{n-1} ; \mathbb{C}, \mathcal{S}\left(\overline{\mathbb{R}}_{+}\right)\right)$with the $(y, \eta)$-wise adjoint in the sense

$$
\left(g_{21, l}(y, \eta) v, c\right)_{\mathbb{C}}=\left(v, g_{21, l}^{*}(y, \eta) c\right)_{L^{2}\left(\mathbb{R}_{+}\right)},
$$

for arbitrary $v \in L^{2}\left(\mathbb{R}_{+}\right), c \in \mathbb{C}$, and $g_{22}(y, \eta) \in S_{\mathrm{cl}}^{\mu}\left(\Omega \times \mathbb{R}^{n-1}\right)$.

The definition for arbitrary $j_{ \pm}$is analogous. We call $g_{21}(y, \eta)$ a trace symbol of order $d \in \mathbb{N}$ and $g_{12}(y, \eta)$ a potential symbol.

From the definition it follows altogether that

$$
\boldsymbol{a}(y, \eta) \in S^{\mu}\left(\Omega \times \mathbb{R}^{n-1} ; H^{s}\left(\mathbb{R}_{+}\right) \oplus \mathbb{C}^{j_{-}}, H^{s-\mu}\left(\mathbb{R}_{+}\right) \oplus \mathbb{C}^{j_{+}}\right)
$$

for all $s>d-\frac{1}{2}$. For $\boldsymbol{g}(y, \eta):=\left(g_{i j}(y, \eta)\right)_{i, j=1,2}$ we have

$$
\boldsymbol{g}(y, \eta) \in S_{\mathrm{cl}}^{\mu}\left(\Omega \times \mathbb{R}^{n-1} ; H^{s}\left(\mathbb{R}_{+}\right) \oplus \mathbb{C}^{j_{-}}, \mathcal{S}\left(\overline{\mathbb{R}}_{+}\right) \oplus \mathbb{C}^{j_{+}}\right)
$$

for $s>d-\frac{1}{2}$. Let $\mathcal{R}_{G}^{\mu, d}\left(\Omega \times \mathbb{R}^{n-1} ; j_{-}, j_{+}\right)$denote the set of all such $\boldsymbol{g}(y, \eta)$. Moreover, let $\mathcal{R}^{\mu, d}\left(\Omega \times \mathbb{R}^{n-1} ; j_{-}, j_{+}\right)$denote the set of all symbols $\boldsymbol{a}(y, \eta)$ of the form (4.23).

Now let $X$ be a $C^{\infty}$ manifold with boundary $Y$. Let us define $\mathcal{B}^{-\infty, d}\left(X ; j_{-}, j_{+}\right)$ to be the space of smoothing operators of type $d$. For simplicity let again $j_{-}=$ $j_{+}=1$ (the general case is analogous).

Based on the Riemannian metrics on $X$ and $Y=\partial X$ we identify the spaces $C^{\infty}(X \times X), C^{\infty}(X \times Y)$, etc., with corresponding integral operators with such kernels, for instance, $u \rightarrow \int_{X} c\left(x, x^{\prime}\right) u\left(x^{\prime}\right) d x^{\prime}$ and $v \rightarrow \int_{Y} k\left(x, y^{\prime}\right) v\left(y^{\prime}\right) d y^{\prime}$ for 
$c\left(x, x^{\prime}\right) \in C^{\infty}(X \times X)$ and $k\left(x, y^{\prime}\right) \in C^{\infty}(X \times Y)$, respectively. Let $\mathcal{B}^{-\infty, 0}(X ; 1,1)$ denote the space of all operators

$$
\mathcal{C}=\left(C_{i j}\right)_{i, j=1,2}: \underset{C_{0}^{\infty}(Y)}{C_{0}^{\infty}(X)} \rightarrow \underset{C^{\infty}(Y)}{C^{\infty}(X)}
$$

such that $C_{11}$ has a kernel in $C^{\infty}(X \times X), C_{12}$ a kernel in $C^{\infty}(X \times Y), C_{21}$ a kernel in $C^{\infty}(Y \times X)$ and $C_{22}$ a kernel in $C^{\infty}(Y \times Y)$. Moreover, by $\mathcal{B}^{-\infty, d}(X ; 1,1)$ for $d \in \mathbb{N}$ we denote the space of all $2 \times 2$ block matrix operators $\mathcal{C}$ where $C_{12}$ and $C_{22}$ are as before but

$$
C_{11}=\sum_{l=0}^{d} C_{11, l} D^{l}, C_{21}=\sum_{l=0}^{d} C_{21, l} D^{l}
$$

for $C_{11, l}$ and $C_{21, l}$ as in the case $d=0$ and a first order differential operator $D$ on $X$ that is close to $Y$ equal to $\partial_{t}$, the differentiation in normal direction. In an analogous manner we define $\mathcal{B}^{-\infty, d}\left(X ; j_{-}, j_{+}\right)$for arbitrary $j_{ \pm} \in \mathbb{N}$.

Let us fix a collar neighbourhood $V$ of $Y$ in $X$, and let $\left(U_{\iota}\right)_{\iota \in I}$ be a locally finite open covering of $V$, and let $\chi_{\iota}: U_{\iota} \rightarrow \overline{\mathbb{R}}_{+}^{n}$ be charts, $\iota \in I$. Those induce charts $\chi_{\iota}^{\prime}: U_{\iota} \cap Y \rightarrow \mathbb{R}^{n-1}$ on $Y$. For every $\boldsymbol{a}_{\iota}(y, \eta) \in \mathcal{R}^{\mu, d}\left(\mathbb{R}^{n-1} \times \mathbb{R}^{n-1} ; j_{-}, j_{+}\right)$ we have an operator $\operatorname{Op}_{y}\left(\boldsymbol{a}_{\iota}\right)$, and we form the pull back $\operatorname{diag}\left(\chi_{*}^{-1}, \chi_{*}^{\prime-1}\right) \operatorname{Op}_{y}\left(\boldsymbol{a}_{\iota}\right)$ which is a $2 \times 2$ block matrix operator over $U_{\iota}$. Let us fix a system of functions $\varphi_{\iota} \in C_{0}^{\infty}\left(U_{\iota}\right)$ such that $\sum_{\iota \in I} \varphi_{\iota} \equiv 1$ near $Y$, set $\varphi_{\iota}:=\left.\varphi_{\iota}\right|_{Y}$, moreover, choose $\psi_{\iota} \in C_{0}^{\infty}\left(U_{\iota}\right)$ that are equal to 1 on $\operatorname{supp} \varphi_{\iota}$, set $\psi_{\iota}^{\prime}=\left.\psi_{\iota}\right|_{Y}$, and form

$$
\mathcal{A}_{\iota}:=\operatorname{diag}\left(\varphi_{\iota}, \varphi_{\iota}^{\prime}\right) \operatorname{diag}\left(\chi_{*}^{-1}, \chi_{*}^{\prime-1}\right) \operatorname{Op}_{y}\left(\boldsymbol{a}_{\iota}\right) \operatorname{diag}\left(\psi_{\iota}, \psi_{\iota}^{\prime}\right)
$$

Moreover, choose functions $\sigma, \tilde{\sigma}, \tilde{\tilde{\sigma}} \in C_{0}^{\infty}(V)$, that are equal to 1 close to $Y$, such that $\tilde{\sigma}=1$ on $\operatorname{supp} \sigma$, and $\sigma \equiv 1$ on $\operatorname{supp} \tilde{\tilde{\sigma}}$.

Definition 4.15. Let $\mathcal{B}^{\mu, d}\left(X ; j_{-}, j_{+}\right)$for $\mu \in \mathbb{Z}, d \in N$, denote the space of all operators

$$
\mathcal{A}=\left(A_{i j}\right)_{i, j=1,2}: \underset{\oplus}{C^{\infty}\left(Y, \mathbb{C}^{j-}\right)} \rightarrow \begin{gathered}
C^{\infty}(X) \\
C^{\infty}\left(Y, \mathbb{C}^{j_{+}}\right)
\end{gathered}
$$

of the form

$$
\mathcal{A}=\operatorname{diag}(\sigma, 1) \sum_{\iota \in I} \mathcal{A}_{\iota} \operatorname{diag}(\tilde{\sigma}, 1)+\operatorname{diag}((1-\sigma) A(1-\tilde{\tilde{\sigma}}), 0)+\mathcal{C}
$$

for arbitrary operators $\mathcal{A}_{\iota}$ as in (4.26), $A \in L_{\mathrm{cl}}^{\mu}(\operatorname{int} X)$, and $\mathcal{C} \in \mathcal{B}^{-\infty, d}\left(X ; j_{-}, j_{+}\right)$.

The definition applies, in particular to $X=\overline{\mathbb{R}}_{+}^{n}$ with the variables $x=(y, t)$. In this case the shape of the operators is easier, since the sum on the right hand side of (4.27) can be replaced by

$$
\operatorname{diag}(\sigma, 1) \operatorname{Op}(\boldsymbol{a}) \operatorname{diag}(\tilde{\sigma}, 1)
$$

for an $\boldsymbol{a}(y, \eta) \in \mathcal{R}^{\mu, d}\left(\mathbb{R}^{n-1} \times \mathbb{R}^{n-1} ; j_{-}, j_{+}\right)$. 
Let us define the principal symbolic structure

$$
\sigma(\mathcal{A})=\left(\sigma_{\psi}(\mathcal{A}), \sigma_{\partial}(\mathcal{A})\right)
$$

consisting of the interior and the boundary symbol $\sigma_{\psi}(\mathcal{A})$ and $\sigma_{\partial}(\mathcal{A})$, respectively.

The upper left corner $A_{11}$ of an operator $\mathcal{A} \in \mathcal{B}^{\mu, d}\left(X ; j_{-}, j_{+}\right)$belongs to $L_{\mathrm{cl}}^{\mu}(\operatorname{int} X)$, and we simply define $\sigma_{\psi}(\mathcal{A})$ for $(x, \xi) \in T^{*} X \backslash 0$ as the homogeneous principal symbol of $A$ of order $\mu$ in the standard sense (here we take into account that the symbols are smooth up to the boundary). What concerns the boundary symbol we first look at the situation of the half-space, cf. (4.28). In this case we define

$$
\sigma_{\partial}(\mathcal{A})(y, \eta):=\sigma_{\partial}(\boldsymbol{a})(y, \eta)
$$

for $(y, \eta) \in T^{*} \mathbb{R}^{n-1} \backslash 0$ by

$$
\sigma_{\partial}(\boldsymbol{a})(y, \eta):=\operatorname{diag}\left(\mathrm{op}^{+}\left(\left.p_{(\mu)}\right|_{t=0}\right)(y, \eta), 0\right)+\sigma_{\partial}(g)(y, \eta)
$$

where $p_{(\mu)}(y, t, \eta, \tau)$ is the homogeneous principal symbol of $p(y, t, \eta, \tau)$, and $\sigma_{\partial}(g)(y, \eta)$ is the homogeneous principal symbol of (4.25) as a classical operatorvalued symbol. Together with

$$
\mathrm{op}^{+}\left(\left.p_{(\mu)}\right|_{t=0}\right)(y, \lambda \eta)=\kappa_{\lambda} \mathrm{op}^{+}\left(\left.p_{(\mu)}\right|_{t=0}\right)(y, \eta) \kappa_{\lambda}^{-1}
$$

for $\lambda \in \mathbb{R}_{+}$we obtain

$$
\sigma_{\partial}(\mathcal{A})(y, \lambda \eta)=\lambda^{\mu} \operatorname{diag}\left(\kappa_{\lambda}, 1\right) \sigma_{\partial}(\mathcal{A})(y, \eta) \operatorname{diag}\left(\kappa_{\lambda}^{-1}, 1\right)
$$

for all $\lambda \in \mathbb{R}_{+}$.

The construction of the operator spaces $\mathcal{B}^{\mu, d}\left(X ; j_{-}, j_{+}\right)$in terms of local representations and subsequent pull backs to the manifold is possible because of natural invariance properties under coordinate changes. The same is true of the principal symbols, and then we obtain, in particular, also an invariantly defined principal boundary symbol on a manifold with boundary, using the local descriptions (4.29). In other words we have

$\sigma_{\psi}(\mathcal{A}) \in C^{\infty}\left(T^{*} X \backslash 0\right), \sigma_{\partial}(\mathcal{A}) \in C^{\infty}\left(T^{*} Y \backslash 0, \mathcal{L}\left(H^{s}\left(\mathbb{R}_{+}\right) \oplus \mathbb{C}^{j_{-}}, H^{s-\mu}\left(\mathbb{R}_{+}\right) \oplus \mathbb{C}^{j_{+}}\right)\right)$.

In many contexts it is adequate to admit operators between sections of smooth complex vector bundles $E, F$ on $X$ and $J_{-}, J_{+}$on $Y$, respectively,

$$
\mathcal{A}: \underset{C_{0}^{\infty}\left(Y, J_{-}\right)}{C_{0}^{\infty}(X, E)} \rightarrow \begin{gathered}
\oplus \\
C^{\infty}\left(Y, J_{-}\right)
\end{gathered} .
$$

The generalisation of the scalar case in the upper left corner to systems and then to the case of bundles, and $E, F$ of the other entries from trivial to general vector bundles $J_{-}, J_{+}$is straightforward and left to the reader.

If $M$ is a $C^{\infty}$ manifold by $\operatorname{Vect}(M)$ we denote the set of all smooth complex vector bundles over $M$. If $M$ is $C^{\infty}$ with boundary then we assume that every $E \in \operatorname{Vect}(M)$ is the restriction of some $\widetilde{E} \in \operatorname{Vect}(2 M)$ to $M$. Then there is a 
standard definition of Sobolev spaces of distributional sections in $E \in \operatorname{Vect}(M)$ in comp/loc-version denoted by

$$
H_{\mathrm{comp} / \mathrm{loc}}^{s}(M, E), s \in \mathbb{R},
$$

when $M$ is an open manifold. If $M$ is compact then we simply write $H^{s}(M, E)$. Moreover, if $M$ is $C^{\infty}$ with boundary we define

$$
H_{\text {[comp } /[\text { loc })}^{s}(\operatorname{int} M, E):=\left.H_{\text {comp } / \text { loc }}^{s}(2 M, \widetilde{E})\right|_{\operatorname{int} M} .
$$

For the vector bundles $E, F \in \operatorname{Vect}(X), J_{-}, J_{+} \in \operatorname{Vect}(Y)$, in (4.31) we write $\boldsymbol{v}:=\left(E, F ; J_{-}, J_{+}\right)$and denote by $\mathcal{B}^{\mu, d}(X ; \boldsymbol{v})$ the set of all operators (4.31).

From the vector-valued analogue of (4.24) together with Theorem 4.7 and corresponding invariance properties we obtain that every $\mathcal{A} \in \mathcal{B}^{\mu, d}(X ;, \boldsymbol{v})$ induces continuous operators

$$
\mathcal{A}: \begin{aligned}
& H_{[\text {comp })}^{s}(\operatorname{int} X, E) \\
& H_{\text {comp }}^{s}\left(Y, J_{-}\right)
\end{aligned} \rightarrow \begin{gathered}
H_{[\text {loc })}^{s-\mu}(\operatorname{int} X, F) \\
\oplus \\
H_{\text {loc }}^{s-\mu}\left(Y, J_{+}\right)
\end{gathered}
$$

for all real $s>d-\frac{1}{2}$. In particular, if $X$ is compact we have

$$
\mathcal{A}: \begin{gathered}
H^{s}(\operatorname{int}(X, E) \\
H^{s}\left(Y, J_{-}\right)
\end{gathered} \rightarrow \begin{gathered}
\oplus \\
H^{s-\mu}(\operatorname{int} X, F) \\
H^{s-\mu}\left(Y, J_{+}\right)
\end{gathered} .
$$

The pair of principal symbols $\sigma=\left(\sigma_{\psi}, \sigma_{\partial}\right)$ in this case means

$$
\sigma_{\psi}(\mathcal{A})(x, \xi): \pi_{X}^{*} E \rightarrow \pi_{X}^{*} F
$$

with the pull back $\pi_{X}^{*}$ of bundles under the canonical projection $\pi_{X}: T^{*} X \backslash 0 \rightarrow X$, and

$$
\sigma_{\partial}(\mathcal{A})(y, \eta): \pi_{Y}^{*}\left(\begin{array}{c}
H^{s}\left(\mathbb{R}_{+}\right) \otimes E^{\prime} \\
\oplus \\
J_{-}
\end{array}\right) \rightarrow \pi_{Y}^{*}\left(\begin{array}{c}
H^{s-\mu}\left(\mathbb{R}_{+}\right) \otimes F^{\prime} \\
\oplus \\
J_{+}
\end{array}\right)
$$

for $E^{\prime}:=\left.E\right|_{Y}, F^{\prime}:=\left.F\right|_{Y}$ and the canonical projection $\pi_{Y}: T^{*} Y \backslash 0 \rightarrow Y$, for $s>d-\frac{1}{2}$. Alternatively we may consider

$$
\sigma_{\partial}(\mathcal{A})(y, \eta): \pi_{Y}^{*}\left(\begin{array}{c}
\mathcal{S}\left(\overline{\mathbb{R}}_{+}\right) \otimes E^{\prime} \\
\oplus \\
J_{-}
\end{array}\right) \rightarrow \pi_{Y}^{*}\left(\begin{array}{c}
\mathcal{S}\left(\overline{\mathbb{R}}_{+}\right) \otimes F^{\prime} \\
\oplus \\
J_{+}
\end{array}\right) .
$$

In the following we often discuss operators in the set-up of $\mathcal{B}^{\mu, d}(X ; \boldsymbol{v})$, though the reader who is mainly interested in the analytical details may consider the case $\mathcal{B}^{\mu, d}\left(X ; j_{-}, j_{+}\right)$which corresponds to the trivial bundles $E=X \times \mathbb{C}, F=X \times \mathbb{C}$ and $J_{ \pm}=Y \times \mathbb{C}^{j_{ \pm}}$, respectively.

Remark 4.16. Let $X$ be compact. Then $\sigma(\mathcal{A})=0$ implies that $(4.32)$ is a compact operator for every $s>d-\frac{1}{2}$. 


\section{Ellipticity of boundary value problems}

We now turn to the ellipticity of BVPs, more precisely, to the Shapiro-Lepatinskij ellipticity. For elliptic operators there is also another kind of ellipticity of boundary conditions, known in special cases, as conditions of Atiyah-Patodi-Singer type ("APS-conditions"), and in general as global projection conditions. While not every elliptic operator on a $C^{\infty}$ manifold $X$ with boundary admits ShapiroLopatinskij elliptic boundary conditions, there are always global projection conditions (when $X$ is compact), see [21] where both concepts are unified to an operator algebra, containing also Boutet de Monvel's calculus. Let $L_{\mathrm{tr}}^{\mu}(X ; E, F)$ for $E, F \in \operatorname{Vect}(X)$ denote the set of all operators $A=\mathrm{r}^{+} \widetilde{A} \mathrm{e}^{+}, \widetilde{A} \in L_{\mathrm{tr}}^{\mu}(2 X ; \widetilde{E}, \widetilde{F})$, with $L_{\mathrm{tr}}^{\mu}(2 X ; \widetilde{E}, \widetilde{F})$ being the space of classical pseudo-differential operators on the double $2 X$, referring to $\widetilde{E}, \widetilde{F} \in \operatorname{Vect}(2 X)$ with $E=\left.\widetilde{E}\right|_{X}, F=\left.\widetilde{F}\right|_{X}$, and with the transmission property at $Y=\partial X$.

For convenience we assume that $Y$ is compact. The nature of elliptic boundary conditions for an elliptic operator $A+G \in \mathcal{B}^{\mu, d}(X ; E, F)$ (i.e., for elliptic $A \in$ $L_{\mathrm{tr}}^{\mu}(X ; E, F), \mu \in \mathbb{Z}$, and a Green operator $G$ on $X$ of order $\mu$ and type $d$ ) depends on the principal boundary symbol of $A$

$$
\sigma_{\partial}(A)(y, \eta): \pi_{Y}^{*} H^{s}\left(\mathbb{R}_{+}\right) \otimes E^{\prime} \rightarrow \pi_{Y}^{*} H^{s-\mu}\left(\mathbb{R}_{+}\right) \otimes F^{\prime}
$$

for any fixed $s>\max \left\{\mu, d-\frac{1}{2}\right\}$, but not so much on

$$
\sigma_{\partial}(G)(y, \eta): \pi_{Y}^{*} H^{s}\left(\mathbb{R}_{+}\right) \otimes E^{\prime} \rightarrow \pi_{Y}^{*} H^{s-\mu}\left(\mathbb{R}_{+}\right) \otimes F^{\prime} .
$$

(5.2) is a family of compact operators that cannot affect the possibility to pose Shapiro-Lopatinskij elliptic conditions for the operator $A$.

Definition 5.1. An operator $\mathcal{A} \in \mathcal{B}^{\mu, d}(X ; \boldsymbol{v})$ for $\boldsymbol{v}=\left(E, F ; J_{-}, J_{+}\right)$is called elliptic if both the principal interior symbol

$$
\sigma_{\psi}(\mathcal{A}): \pi_{X}^{*} E \rightarrow \pi_{X}^{*} F
$$

$\pi_{X}: T^{*} X \backslash 0 \rightarrow X$, and the principal boundary symbol

$$
\sigma_{\partial}(\mathcal{A}): \pi_{Y}^{*}\left(\begin{array}{c}
H^{s}\left(\mathbb{R}_{+}\right) \otimes E^{\prime} \\
\oplus \\
J_{-}
\end{array}\right) \rightarrow \pi_{Y}^{*}\left(\begin{array}{c}
H^{s-\mu}\left(\mathbb{R}_{+}\right) \otimes F^{\prime} \\
\oplus \\
J_{+}
\end{array}\right)
$$

$\pi_{Y}: T^{*} Y \backslash 0 \rightarrow Y$, define isomorphisms.

The second condition is just what we call Shapiro-Lopatinskij ellipticity. The smoothness $s>\max \{\mu, d\}-\frac{1}{2}$ is fixed, but the choice is unessential. The bijectivity of $\sigma_{\partial}(\mathcal{A})$ holds if and only if its restriction to Schwartz functions in the upper left corner induces an isomorphism

$$
\sigma_{\partial}(\mathcal{A}): \pi_{Y}^{*}\left(\begin{array}{c}
\mathcal{S}\left(\overline{\mathbb{R}}_{+}\right) \otimes E^{\prime} \\
\oplus \\
J_{-}
\end{array}\right) \rightarrow \pi_{Y}^{*}\left(\begin{array}{c}
\mathcal{S}\left(\overline{\mathbb{R}}_{+}\right) \otimes F^{\prime} \\
\oplus \\
J_{+}
\end{array}\right)
$$


If $\mathcal{A} \in \mathcal{B}^{\mu, d}(X ; \boldsymbol{v}), \boldsymbol{v}=\left(E, F ; J_{-}, J_{+}\right)$, is elliptic in the sense of Definition 5.1, then for the pair of inverses $\sigma_{\psi}^{-1}(\mathcal{A})$ and $\sigma_{\partial}^{-1}(\mathcal{A})$ we find an operator $\mathcal{A}^{(-1)} \in$ $\mathcal{B}^{-\mu,(d-\mu)^{+}}\left(X ; \boldsymbol{v}^{-1}\right), \boldsymbol{v}^{-1}=\left(F, E ; J_{+}, J_{-}\right),(d-\mu)^{+}=\max \{d-\mu, 0\}$, such that $\sigma_{\psi}\left(\mathcal{A}^{(-1)}\right)=\sigma_{\psi}^{-1}(\mathcal{A}), \sigma_{\partial}\left(\mathcal{A}^{(-1)}\right)=\sigma_{\partial}^{-1}(\mathcal{A})$. This is a consequence of a more general operator convention to find operators to a prescribed pair of principal symbols (those can be described independently of the operator level, similarly as in the case of classical pseudo-differential operators on an open manifold).

In that case we have compact remainders

$$
\begin{aligned}
\mathcal{G} & :=1-\mathcal{A}^{(-1)} \mathcal{A} \in \mathcal{B}^{-1, \max \{\mu, d\}}\left(X ;\left(E, E ; J_{-}, J_{-}\right)\right), \\
\mathcal{D} & :=1-\mathcal{A A}^{(-1)} \in \mathcal{B}^{-1,(d-\mu)^{+}}\left(X ;\left(F, F ; J_{+}, J_{+}\right)\right)
\end{aligned}
$$

in the respective Sobolev spaces, since $\sigma(\mathcal{G})=0, \sigma(\mathcal{D})=0$, (with $\sigma$ referring to order 0 ). This shows the first part of the following theorem.

Theorem 5.2. Let $\mathcal{A} \in \mathcal{B}^{\mu, d}(X ; \boldsymbol{v})$ be elliptic; then

$$
\mathcal{A}: \begin{aligned}
& H^{s}(\operatorname{int} X, E) \\
& H^{s}\left(Y, J_{-}\right)
\end{aligned} \rightarrow \begin{gathered}
H^{s-\mu}(\operatorname{int} X, F) \\
H^{s-\mu}\left(Y, J_{+}\right)
\end{gathered}
$$

is a Fredholm operator for every $s>\max \{\mu, d\}-\frac{1}{2}$. Conversely, if (5.7) is Fredholm for some $s=s_{0}>\max \{\mu, d\}-\frac{1}{2}$, then $\mathcal{A}$ is elliptic which entails the Fredholm property for all $s>\max \{\mu, d\}-\frac{1}{2}$.

The second part of the latter theorem requires arguments that are omitted here; details may be found in [15].

Remark 5.3. $\quad$ 1. If $\mathcal{A} \in \mathcal{B}^{\mu, d}(X, \boldsymbol{v})$ is elliptic then there is a parametrix $\mathcal{A}^{(-1)} \in$ $\mathcal{B}^{-\mu,(d-\mu)^{+}}\left(X ; \boldsymbol{v}^{-1}\right)$ which means that the above-mentioned remainders $\mathcal{G}$ and $\mathcal{D}$ belong to $\mathcal{B}^{-\infty, \max }\{\mu, d\}$ and $\mathcal{B}^{-\infty,(d-\mu)^{+}}$, respectively.

2. Let $\mathcal{A} \in \mathcal{B}^{\mu, d}(X ; \boldsymbol{v})$ be an operator such that (5.7) is an isomorphism for some $s=s_{0}>\max \{\mu, d\}-\frac{1}{2}$. Then (5.7) is an isomorphism for all $s>\max \{\mu, d\}-\frac{1}{2}$, and for the inverse (which is a special parametrix of $\mathcal{A})$ we have $\mathcal{A}^{-1} \in \mathcal{B}^{-\mu,(d-\mu)^{+}}\left(X ; \boldsymbol{v}^{-1}\right)$.

In fact, 1 . can be obtained by improving $\mathcal{A}^{(-1)}$ of $(5.5),(5.6)$ by applying a formal Neumann series argument. The property 2. is a consequence of the second assertion of Theorem 5.2 (more details may be found in [24]).

Example. Let $A=\Delta$, the Laplacian on $X$ (with respect to a Riemannian metric), moreover, let $T_{0} u:=\left.u\right|_{Y}$. Then, for every order reducing isomorphism $R \in L_{\mathrm{cl}}^{3 / 2}(Y)$ on the boundary we have

$$
\left(\begin{array}{l}
\Delta \\
T
\end{array}\right):=\left(\begin{array}{cc}
1 & 0 \\
0 & R
\end{array}\right)\left(\begin{array}{c}
\Delta \\
T_{0}
\end{array}\right) \in \mathcal{B}^{2,0}(X ; 1,1 ; 0,1)
$$


where 1 on the right hand side stands for trivial bundles of fibre dimension 1 over $X$ and $Y$, respectively, and we have

$$
\left(\begin{array}{l}
\Delta \\
T
\end{array}\right)^{-1} \in \mathcal{B}^{-2,0}(X ; 1,1 ; 1,0) .
$$

Let us now discuss the nature of Shapiro-Lopatinskij ellipticity in more detail. A closer look at (5.1) reveals some interesting structures that are useful also to understand the difference to ellipticity with global projection conditions, mentioned at the beginning of this section.

Consider an operator $A \in \mathcal{B}^{\mu, 0}(X ; E, F)$ (i.e., $A$ is of the type of an upper left corner in the $2 \times 2$ block matrix set-up) satisfying the ellipticity condition (5.3). Then (5.1) is a family of Fredholm operators, where dim $\operatorname{ker} \sigma_{\partial}(A)(y, \eta)$ and dim coker $\sigma_{\partial}(A)(y, \eta)$ are independent of $s>\max \{\mu, d\}-\frac{1}{2}$. The same is true of

$$
\sigma_{\partial}(\mathcal{A})(y, \eta)_{1,1}=\sigma_{\partial}(A)(y, \eta)+\sigma_{\partial}(G)(y, \eta) .
$$

If $(5.4)$ is a family of isomorphisms then the role of the additional entries $\left(\sigma_{\partial}(\mathcal{A})(y, \eta)\right)_{i, j}$ for $i+j>2$ is to fill up (5.8) to a family of isomorphisms. However, many operators $A \in \mathcal{B}^{\mu, 0}(X ; E, F)$ that are elliptic with respect to $\sigma_{\psi}(\cdot)$ do not admit such families of block matrix isomorphisms.

As noted before an example is the Cauchy-Riemann operator in a smooth bounded domain in $\mathbb{C}$ which is elliptic of order 1 . Other examples are Dirac operators in even dimensions.

In order to illustrate the phenomenon in general we recall a few notions from $K$-theory which are connected with the index of families of Fredholm operators parametrised by a compact topological space. In the present case we consider $(5.8)$ for $(y, \eta) \in S^{*} Y$, the unit cosphere bundle induced, by $T^{*} Y \backslash 0$. Observe that by virtue of the homogeneity

$$
\left(\sigma_{\partial}(\mathcal{A})(y, \lambda \eta)\right)_{1,1}=\lambda^{\mu} \kappa_{\lambda}\left(\sigma_{\partial}(\mathcal{A})(y, \eta)_{1,1}\right) \kappa_{\lambda}^{-1}
$$

the values of $\sigma_{\partial}(\mathcal{A})(y, \eta)_{1,1}$ for all $(y, \eta) \in T^{*} Y \backslash 0$ are determined by those for $(y, \eta) \in S^{*} Y$. The compact topological spaces that we have in mind here are $S^{*} Y$ and $Y$, respectively (we discuss the case that $X$ is a smooth manifold with compact boundary $Y$ ).

First, on a compact topological space $M$ (for simplicity connected) we have the set $\operatorname{Vect}(M)$ of (locally trivial) continuous complex vector bundles on $M$. In the case of a $C^{\infty}$ manifold $M$ we may (and will) take smooth complex vector bundles. Roughly speaking, continuous vector bundles over $M$ are topological spaces which are disjoint unions $E=\bigcup_{x \in M} E_{x}$ of fibres $E_{x}$ that are vector spaces isomorphic to $\mathbb{C}^{k}$ for some $k \in N$, and every point $x_{0} \in M$ has a neighbourhood $U$ such that $\left.E\right|_{U}=\bigcup_{x \in U} E_{x}$ is homeomorphic to $U \times \mathbb{C}^{k}$ where this homorphism is fibrewise an isomorphism and commutes with the canonical projections $p: E \rightarrow M, e_{x} \rightarrow x$ for $e_{x} \in E_{x}$, and $q: U \times \mathbb{C}^{k} \rightarrow U,(x, v) \rightarrow x$ for $v \in \mathbb{C}^{k}$. An example is $E=M \times \mathbb{C}^{k}$ which is a so-called trivial vector bundle. Thus a part of the general definition requires $\left.E\right|_{U}$ to be isomorphic to a trivial bundle which is just the meaning of 
"locally trivial". We do not repeat here everything on vector bundles such as what is a vector bundle isomorphism $\cong$, but the notion directly comes from vector space isomorphisms, now parametrised by $x \in M$. More generally, we have vector bundle morphisms which are fibrewise vector space homomorphisms. Moreover, we have a natural notion of a direct sum $E \oplus F$ for $E, F \in \operatorname{Vect}(M)$, fibrewise defined by $E_{x} \oplus F_{x}, x \in M$.

Similarly we can form tensor products $E \otimes F$ by taking fibrewise tensor products $E_{x} \otimes F_{x}, x \in M$.

The $K$-group $K(M)$ over $M$ is defined as the set of equivalence classes of pairs $(E, F) \in \operatorname{Vect}(M) \times \operatorname{Vect}(M)$ where

$$
(E, F) \sim(\widetilde{E}, \widetilde{F})
$$

means that there is a $G \in \operatorname{Vect}(M)$ such that $E \oplus \widetilde{F} \oplus G \cong F \oplus \widetilde{E} \oplus G$. The equivalence class represented by $(E, F)$ is denoted by $[E]-[F]$. The structure of $K(M)$ of a commutative group comes from the direct sum, namely,

$$
\left(\left[E_{1}\right]-\left[F_{1}\right]\right)+\left(\left[E_{2}\right]-\left[F_{2}\right]\right):=\left[E_{1} \oplus E_{2}\right]-\left[F_{1} \oplus F_{2}\right] .
$$

Note that the tensor product between bundles turns $K(M)$ even to a commutative ring.

Moreover, recall that when $f: M \rightarrow N$ is a continuous map we have the bundle pull back $E \rightarrow f^{*} E$ for $E \in \operatorname{Vect}(N)$ and a resulting $f^{*} E \in \operatorname{Vect}(M)$. This gives rise to a homomorphism

$$
f^{*}: K(N) \rightarrow K(M)
$$

defined by $f^{*}([E]-[F])=\left[f^{*} E\right]-\left[f^{*} F\right]$.

An example is $M:=S^{*} Y, N=Y$, with the canonical projection,

$$
\pi_{1}: S^{*} Y \rightarrow Y, \pi_{1}(y, \eta)=y .
$$

(Non-trivial) vector bundles may appear in connection with elliptic boundary value problems, or, more generally, with families of Fredholm operators. The latter ones give rise to an equivalent definition of $K(M)$. The construction is closely related to the task to find entries $\sigma_{\partial}(\mathcal{A})(y, \eta)_{i, j}$ for $i, j=1,2, i+j>2$, for a given $\sigma_{\partial}(\mathcal{A})(y, \eta)_{1,1}$ that complete the latter Fredholm family to a family of isomorphisms, cf. (5.4). The general construction is as follows.

By $\mathcal{F}(H, \widetilde{H})$ for Hilbert spaces $H, \widetilde{H}$ we denote the set of all Fredholm operators $H \rightarrow \widetilde{H}$. Recall that $\mathcal{F}(H, \widetilde{H})$ is open in $\mathcal{L}(H, \widetilde{H})$, the space of all linear continuous operators in the operator norm topology.

Lemma 5.4. Let $a \in C(M, \mathcal{F}(H, \widetilde{H}))$, and assume that $a(x): H \rightarrow \widetilde{H}$ is surjective for every $x \in M$. Then the family of kernels

$$
\operatorname{ker}_{M} a:=\{\operatorname{ker} a(x): x \in M\}
$$

has the structure of a (continuous) vector bundle over $M$. 
Proof. Let $\pi: H \rightarrow \operatorname{ker} a\left(x_{1}\right)$ be the orthogonal projection to ker $a\left(x_{1}\right)$ for any fixed $x_{1} \in M$. Then the family of continuous operators

$$
\left(\begin{array}{c}
a(x) \\
\pi\left(x_{1}\right)
\end{array}\right): H \rightarrow \begin{gathered}
\widetilde{H} \\
\operatorname{ker} a\left(x_{1}\right)
\end{gathered}
$$

is an isomorphism at $x=x_{1}$ and hence for all $x$ in an open neighbourhood $U$ of $x_{1}$. Therefore, by virtue of Lemma 2.3 the operator $\pi\left(x_{1}\right)$ induces isomorphisms $\pi\left(x_{1}\right): \operatorname{ker} a(x) \rightarrow \operatorname{ker} a\left(x_{1}\right)$ for all $x \in U$. This gives us a continuous family of maps

$$
\{\operatorname{ker} a(x): x \in U\} \rightarrow U \times \operatorname{ker} a\left(x_{1}\right) .
$$

which is just the desired trivialisation when we identify $\operatorname{ker} a\left(x_{1}\right)$ with $\mathbb{C}^{k}$ for $k=\operatorname{dim} \operatorname{ker} a\left(x_{1}\right)$.

Lemma 5.5. For every $a \in C(M, \mathcal{F}(H, \widetilde{H}))$ there exists a $j_{-} \in \mathbb{N}$ and a linear operator ker : $\mathbb{C}^{j_{-}} \rightarrow \widetilde{H}$ such that

$$
\left(\begin{array}{lll}
a(x) & k
\end{array}\right): \underset{\mathbb{C}^{j_{-}}}{\stackrel{H}{\oplus}} \rightarrow \widetilde{H}
$$

is surjective for every $x \in M$.

Proof. For every $x_{1} \in M$ there exists a finite-dimensional subspace $W_{1} \subset \widetilde{H}$ and an isomorphism $k_{1}: \mathbb{C}^{j_{1}} \rightarrow W_{1}$ for $j_{1}=\operatorname{dim} W_{1}$ such that

$$
\left(\begin{array}{ll}
a(x) & k_{1}
\end{array}\right): \underset{\mathbb{C}^{j_{1}}}{\bigoplus} \rightarrow \widetilde{H}
$$

is surjective for $x=x_{1}$. Then (5.12) is surjective for all $x \in U_{1}$ for some open neighbourhood $U_{1}$ of $x_{1}$. Those neighbourhoods, parametrised by $x_{1} \in M$, form an open covering of $M$. Since $M$ is compact, there are finitely many points $x_{1}, \ldots, x_{N} \in M$ such that $M=\bigcup_{l=1}^{N} U_{l}$ for the respective $U_{l}$. Choosing operators $k_{l}$ analogously as in (5.12) for every $1 \leq l \leq 1$, with dimensions $j_{l}$ rather than $j_{1}$, we obtain the assertion for $k:=\left(k_{1}, \ldots, k_{N}\right)$, and $j_{-}:=\sum_{l=1}^{N}$.

Proposition 5.6. For every $a \in C(M, \mathcal{F}(H, \widetilde{H}))$ there exist vector bundles $J_{-}, J_{+} \in$ $\operatorname{Vect}(M)$ and a continuous family of isomorphisms

$$
\boldsymbol{a}:=\left(\begin{array}{ll}
a(x) & k(x) \\
t(x) & q(x)
\end{array}\right) \underset{J_{-, x}}{\oplus} \underset{J_{+, x}}{\oplus} \underset{\tilde{H}}{\oplus}, \quad x \in M
$$

Proof. Choose $k=k(x)$ as in Lemma 5.5 for the trivial bundle $J_{-}=M \times \mathbb{C}^{j_{-}}$. Then applying Lemma 5.4 to the Fredholm family (5.11) we obtain that

$$
\operatorname{ker}_{M}(a(x) \quad k)
$$


is a finite-dimensional subbundle of ${ }^{\mathrm{t}}\left(H \oplus \mathbb{C}^{j_{-}}\right)$, isomorphic to $J_{+}$for some $J_{+} \in$ $\operatorname{Vect}(M)$. Choosing a bundle isomorphism

$$
b_{0}: \operatorname{ker}_{M}(a(x) \quad k) \rightarrow J_{+}
$$

and setting $b:=b_{0} \circ \pi(x)$ for the family of orthogonal projections $\pi(x):{ }^{\mathrm{t}}(H \oplus$ $\left.\mathbb{C}^{j-}\right) \rightarrow \operatorname{ker}(a(x) \quad k)$ we obtain our result when we set

$$
t(x):=\left.b(x)\right|_{M}, q(x):=\left.b(x)\right|_{\mathbb{C}^{j_{-}}} .
$$

Definition 5.7. For $a \in C(M, \mathcal{F}(H, \widetilde{H}))$ and any choice of (5.13) we set

$$
\operatorname{ind}_{M} a:=\left[J_{+}\right]-\left[J_{-}\right],
$$

called the $K$-theoretic index of the Fredholm family $a$.

It can be proved, cf. [10], that $\operatorname{ind}_{M} a$ only depends on $a$ but not on the specific choice of the family of isomorphisms (5.13).

In particular, we obtain the same $\operatorname{ind}_{M} a$ when we replace (5.13) by isomorphisms of the kind

$$
\left(\begin{array}{cc}
\boldsymbol{a}(x) & h(x) \\
b(x) & d(x)
\end{array}\right): \begin{gathered}
\oplus \oplus J_{-, x} \\
L_{-, x}
\end{gathered} \quad \begin{gathered}
\widetilde{H}_{\oplus} \oplus J_{+, x} \\
L_{+, x}
\end{gathered}
$$

for some $L_{-}, L_{+} \in \operatorname{Vect}(M)$. Moreover, if $c \in C(M, \mathcal{L}(H, \widetilde{H}))$ is a family of compact operators, then

$$
\operatorname{ind}_{M}(a+c)=\operatorname{ind}_{M} a .
$$

The map $\operatorname{ind}_{M}: C(M, \mathcal{F}(H, \widetilde{H})) \rightarrow K(M)$ is surjective and induces a map only depending on the homotopy classes of Fredholm families. This gives rise to an equivalent definition of $K(M)$, cf. Jänich [11].

Let $X$ be compact, $E, F \in \operatorname{Vect}(X)$, and let $A \in \mathcal{B}^{\mu, d}(X ;(E, F ; 0,0))$ be elliptic with respect to $\sigma_{\psi}$ (cf. the first condition of Definition 5.1). Then the restriction of $\sigma_{\partial}(A)(y, \eta)$ to $S^{*} Y$ (for brevity denoted again by $\sigma_{\partial}(A)(y, \eta)$ ) gives us a family of Fredholm operators

$$
\sigma_{\partial}(A)(y, \eta): H^{s}\left(\mathbb{R}_{+}\right) \otimes E^{\prime} \rightarrow H^{s-\mu}\left(\mathbb{R}_{+}\right) \otimes F^{\prime},
$$

$s>\max \{\mu, d\}-\frac{1}{2}$, parametrised by $(y, \eta) \in S^{*} Y$. Therefore, we obtain an index element

$$
\operatorname{ind}_{S^{*} Y} \sigma_{\partial}(A) \in K\left(S^{*} Y\right)
$$

(which is independent of $s$ ). The following theorem was first formulated in the case of differential operators in the paper [2] by Atiyah and Bott, and then for pseudo-differential operators with the transmission property at the boundary in [4] by Boutet de Monvel, cf. also [21]. An analogue for edge operators may be found in [17], cf. also the author's joint papers [23], [27], with Seiler, and the references there. 
Theorem 5.8. $A \sigma_{\psi}$-elliptic operator $A \in \mathcal{B}^{\mu, d}(X ;(E, F ; 0,0))$ can be completed by additional entries to a $\left(\sigma_{\psi}, \sigma_{\partial}\right)$-elliptic $2 \times 2$ block matrix operator

$$
\mathcal{A} \in \mathcal{B}^{\mu, d}\left(X ;\left(E, F ; J_{-}, J_{+}\right)\right)
$$

for suitable $J_{-}, J_{+} \in \operatorname{Vect}(Y)$ with $A$ in the upper left corner if and only if

$$
\operatorname{ind}_{S^{*} Y} \sigma_{\partial}(A) \in \pi_{1}^{*} K(Y)
$$

(cf. the notation (5.9)).

Proof. The condition (5.16) is necessary, since the Shapiro-Lopatinskij ellipticity means that (5.4) is a family of isomorphisms and hence, by virtue of (5.14),

$$
\operatorname{ind}_{S^{*} Y} \sigma_{\partial}(A)=\left[\pi_{1}^{*} J_{+}\right]-\left[\pi_{1}^{*} J_{-}\right] .
$$

Conversely, the condition (5.16) allows us to construct a block matrix family of isomorphisms of the kind (5.13) with $\sigma_{\partial}(A)$ in the upper left corner and vector bundles over $S^{*} Y$ that are pull backs of vector bundles over $Y$. The construction for every $(y, \eta) \in S^{*} Y$ is practically the same at that in the proof of Proposition 5.6. In addition we guarantee that the resulting block matrix operators locally belong to $\mathcal{B}^{\mu, d}\left(\overline{\mathbb{R}}_{+} ; k, k ; j_{-}, j_{+}\right)$and smoothly depend on $(y, \eta)$, for $k=\operatorname{dim} E_{y}=\operatorname{dim} F_{y}$, $j_{ \pm}=\operatorname{dim} J_{ \pm, y}$. The corresponding operator functions $k(y, \eta), t(y, \eta)$ and $q(y, \eta)$ can be extended from $S^{*} Y$ to $T^{*} Y \backslash 0$ by $\kappa_{\lambda}$-homogeneity of order $\mu$. This can be done in terms of principal parts of symbols belonging to (4.25). Then applying an operator convention which assigns to such principal symbols associated operators gives us the additional entries.

Remark 5.9. The proof of Theorem 5.8 shows how we can find (in principle all) Shapiro-Lopatinskij elliptic boundary value problems $\mathcal{A} \in \mathcal{B}^{\mu, d}(X ; \boldsymbol{v}), \boldsymbol{v}=$ $\left(E, F ; J_{-}, J_{+}\right)$, for any given $\sigma_{\psi^{-}}$elliptic operator $A \in \mathcal{B}^{\mu, d}(X ;(E, F ; 0,0))$ provided that the topological condition (5.16) is satisfied. It turns out that, from the point of view of the associated Fredholm indices, for every two such $\mathcal{A}_{1}, \mathcal{A}_{2}$ with the same upper left corner we can construct an elliptic operator $R$ on the boundary such that

$$
\operatorname{ind} \mathcal{A}_{1}-\operatorname{ind} \mathcal{A}_{2}=\operatorname{ind} R \text {. }
$$

The latter relation is known as the Agranovich-Dynin formula (see also [4] and [15]). The proof is close to what we did in (2.19) modified for general $2 \times 2$ matrices rather than column matrices, cf. [15, Section 3.2.1.3.].

It may happen that $\sigma_{\partial}(A)(y, \eta)$ is a family of isomorphisms (5.15), i.e., that for the ellipticity of $A$ with respect to $\sigma_{\psi}$ and $\sigma_{\partial}$ no additional entries are necessary. For instance, consider the symbol

$$
r_{-}^{\mu}(\eta, \tau):=\left(\varphi\left(\frac{\tau}{C\langle\eta\rangle}\right)\langle\eta\rangle-i \tau\right)^{\mu}
$$

for some fixed $\varphi(t) \in \mathcal{S}(\mathbb{R})$ such that $\varphi(0)=1$ and $\operatorname{supp} F^{-1} \varphi \subset \mathbb{R}_{-}$, for instance, $\varphi(\tau):=c^{-1} \int_{-\infty}^{0} e^{-i t \tau} \psi(t) d t$ for some $\psi \in C_{0}^{\infty}\left(\mathbb{R}_{-}\right)$where $c:=\int_{-\infty}^{0} \psi(t) d t \neq 0$. Then, if $C>0$ is a sufficiently large constant we have $r_{-}^{\mu}(\eta, \tau) \in S_{\mathrm{tr}}^{\mu}\left(\mathbb{R}^{n}\right)$, and 
$r_{-}^{\mu}(\eta, \tau)$ is elliptic of order $\mu$. This symbol can be smootly connected with $\langle\eta, \tau\rangle^{\mu}$ far from $t=0$ by forming $r_{-}^{\mu \omega(t)}(\eta, \tau)\langle\eta, \tau\rangle^{\mu(1-\omega(t))}$ for a real-valued $\omega \in C_{0}^{\infty}(\mathbb{R})$ such that $\omega \equiv 1$ in a neighbourhood of $t=0$. Then, if we interpret $t \in \overline{\mathbb{R}}_{+}$as the inner normal of a collar neighbourhood of $Y$ in $X$ there is obviously a $\sigma_{\psi}$-elliptic operator $R_{-}^{\mu}$ on $X$ with such amplitude functions near the boundary, and $\sigma_{\partial}\left(R_{-}^{\mu}\right)$ has the desired property, indeed. A similar construction is possible in the vector bundle set-up, which gives us such an operator $R_{-, E}^{\mu} \in \mathcal{B}^{\mu, d}(X ;(E, E ; 0,0))$,

$$
R_{-, E}^{\mu}: H^{s}(\operatorname{int} X, E) \rightarrow H^{s-\mu}(\operatorname{int} X, E) .
$$

In addition the operator convention can be chosen in such a way that (5.17) is an isomorphism for every $s>\max \{\mu, d\}-\frac{1}{2}$. More details on such constructions may be found in the paper [9] of Grubb, see also the author's joint monograph with Harutyuyan [10, Section 4.1].

Using the fact that there are also order reducing operators of any order on the boundary (which is a compact $C^{\infty}$ manifold, cf. the formulas $(2.5),(2.12)$ ) we can compose any $\left(\sigma_{\psi}, \sigma_{\partial}\right)$-elliptic operator $\mathcal{A} \in \mathcal{B}^{\mu, d}\left(X ;\left(E, F ; J_{-}, J_{+}\right)\right)$by diagonal matrices of order reductions to a $\left(\sigma_{\psi}, \sigma_{\partial}\right)$-elliptic operator $\mathcal{A}_{0} \in \mathcal{B}^{0,0}(X ;(E, F$; $\left.\left.J_{-}, J_{+}\right)\right)$. For many purposes it is convenient to deal with operators of order and type zero, and we will assume that for a while, in order to illustrate other interesting aspects of elliptic pseudo-differential boundary value problems.

Let us set

$$
\Xi^{*}:=\left.S^{*} X\right|_{Y} \cup N^{*}
$$

with $\left.S^{*} X\right|_{Y}$ denoting the restriction of the unit cosphere bundle to the boundary, and

$$
N^{*}=\left\{\left.(y, 0,0, \tau) \in T^{*} X\right|_{Y}:-1 \leq \tau \leq 1\right\}
$$

which refers to the splitting of variables $x=(y, t)$ near the boundary. The interval bundle $N^{*}$ is trivial and its fibres $N_{y}^{*}=\{(y, 0,0, \tau):-1 \leq \tau \leq 1\}$ connect the south poles $(\tau=-1)$ with the north poles $(\tau=+1)$ of $\left.S^{*} X\right|_{y}, y \in Y$. In other words, $\Xi^{*}$ is a kind of cage with bars $N_{y}^{*}$, called the conormal cage. Let

$$
\pi_{\mathrm{c}}: \Xi^{*} \rightarrow Y
$$

denote the canonical projection.

Remark 5.10. Let $A \in \mathcal{B}^{0,0}(X ;(E, F ; 0,0))$ be $\sigma_{\psi}$-elliptic; then $\left.\sigma_{\psi}(A) X\right|_{Y}$ extends to an isomorphism

$$
\sigma_{\psi}^{\prime}(A): \pi_{\mathrm{c}}^{*} E^{\prime} \rightarrow \pi_{\mathrm{c}}^{*} F^{\prime} .
$$

In fact, $\sigma_{\psi}(A)(y, 0, \eta, \tau): E_{y} \rightarrow F_{y}$ is a family of isomorphisms for all $\left.(y, 0, \eta, \tau) \in S^{*} X\right|_{Y}$. By virtue of the transmission property we have

$$
\sigma_{\psi}(A)(y, 0,0-1)=\sigma_{\psi}(A)(y, 0,0,+1) .
$$


The principal symbol $\sigma_{\psi}(A)(x, \xi)$ is altogether (positively) homogeneous of order zero in $\xi \neq 0$; in particular, we have

$$
\begin{aligned}
\sigma_{\psi}(A)(y, 0,0, \tau) & =\sigma_{\psi}(A)(y, 0,0,-1) \text { for all } \tau<0, \\
\sigma_{\psi}(A)(y, 0,0, \tau) & =\sigma_{\psi}(A)(y, 0,0,+1) \text { for all } \tau>0 .
\end{aligned}
$$

Now the relation $(5.20)$ shows that $\sigma_{\psi}(A)(y, 0,0, \tau)$ does not depend on $\tau \neq 0$, and hence it extends to $N_{y}^{*}$ when we define

$$
\sigma_{\psi}^{\prime \prime}(A)(y):=\sigma_{\psi}(A)(y, 0,0,0):=\sigma_{\psi}(A)(y, 0,0,1) .
$$

We obtain an isomorphism

$$
\sigma_{\psi}^{\prime \prime}(A): E^{\prime} \rightarrow F^{\prime}
$$

Let us now return to operators on the half-axis

$$
\mathrm{op}^{+}\left(\left.\sigma_{\psi}(A)\right|_{t=0}\right)(y, \eta): L^{2}\left(\mathbb{R}_{+}\right) \otimes E_{y}^{\prime} \rightarrow L^{2}\left(\mathbb{R}_{+}\right) \otimes F_{y}^{\prime}
$$

parametrised by $(y, \eta) \in S^{*} Y$. By virtue of $(5.21)$ we may replace $F^{\prime}$ by $E^{\prime}$. As usual we interpret $\bigcup_{y \in Y} L^{2}\left(\mathbb{R}_{+}\right) \otimes E_{y}^{\prime}$ as $L^{2}\left(\mathbb{R}_{+}\right) \otimes E^{\prime}$ which is a Hilbert space bundle over $Y$ (by Kuiper's theorem it is trivial). Set $a(y, \eta, \tau):=\sigma_{\psi}(A)(y, 0, \eta, \tau)$ which is a family of isomorphisms

$$
a(y, \eta, \tau): E_{y}^{\prime} \rightarrow E_{y}^{\prime}
$$

$\left.(y, \eta, \tau) \in S^{*} X\right|_{Y}$. By virtue of the homogeneity we have $a(y, \lambda \eta, \lambda \tau)=a(y, \eta, \tau)$ for all $\lambda \in \mathbb{R}_{+}$, in particular,

$$
a\left(y, \frac{\eta}{|\tau|}, \frac{\tau}{|\tau|}\right)=a(y, \eta, \tau)
$$

for all $\tau \neq 0$. Thus (5.20) gives us

$$
\lim _{\tau \rightarrow-\infty} a(y, \eta, \tau)=a(y, 0,-1)=a(y, 0,+1)=\lim _{\tau \rightarrow+\infty} a(y, \eta, \tau) .
$$

This fits to the picture of symbols with the transmission property in $\tau$ described in Section 3. In other words, we have

$$
a(y, \eta, \tau) \in S_{\mathrm{tr}}^{0}(\mathbb{R}) \otimes \operatorname{Iso}\left(E_{y}, E_{y}\right)
$$

for every fixed $(y, \eta) \in S^{*} Y$ (here Iso $(\cdot, \cdot)$ means the space of isomorphisms between the vector spaces in parenthesis). The operators

$$
\mathrm{op}^{+}(a)(y, \eta): L^{2}\left(\mathbb{R}_{+}\right) \otimes E_{y} \rightarrow L^{2}\left(\mathbb{R}_{+}\right) \otimes E_{y}
$$

are Fredholm and their pointwise index is equal to the winding number of the curve

$$
L(a):=\{\operatorname{det} a(y, \eta, \tau): \tau \in \mathbb{R}\} \subset \mathbb{C} .
$$

This is a useful information for the construction of extra trace and potential conditions in an elliptic BVP. It would be optimal to know the dimensions of kernel and cokernel; of course, those are not necessarily constant in $y$. 


\section{The anti-transmission property}

In this section we return to scalar symbols (for simplicity). Recall that the transmission property of a symbol $a(\tau) \in S_{\mathrm{cl}}^{\mu}(\mathbb{R})$ means the condition (3.11). In general, the curve

$$
L(a)=\{a(\tau) \in \mathbb{C}: \tau \in \mathbb{R}\}
$$

is not closed. Let $a(y, t, \eta, \tau) \in S_{\mathrm{cl}}^{0}\left(\Omega \times \overline{\mathbb{R}}_{+} \times \mathbb{R}_{\eta, \tau}^{n}\right)$ be an elliptic symbol, $a_{(0)}$ its homogeneous principal part, and $a(\tau):=a_{(0)}(y, 0, \eta, \tau)$ for fixed $(y, \eta) \in T^{*} \Omega \backslash 0$. Then, similarly as in elliptic BVPs with the transmission property, a task is to find a bijective $2 \times 2$ block matrix

$$
\boldsymbol{a}=\left(\begin{array}{cc}
\mathrm{op}^{+}(a) & k \\
b & q
\end{array}\right): \underset{\mathbb{C}^{j_{-}}}{\overbrace{}^{2}\left(\mathbb{R}_{+}\right)} \rightarrow \begin{gathered}
L^{2}\left(\mathbb{R}_{+}\right) \\
\mathbb{C}^{j_{+}}
\end{gathered}
$$

for suitable $j_{ \pm} \in \mathbb{N}$. This is possible if and only if

$$
\mathrm{op}^{+}(a): L^{2}\left(\mathbb{R}_{+}\right) \rightarrow L^{2}\left(\mathbb{R}_{+}\right)
$$

is a Fredholm operator. Set

$$
M(a):=\left\{z \in \mathbb{C}: z=(1-\lambda) a_{0}^{+}+\lambda a_{0}^{-}, 0 \leq \lambda \leq 1\right\} .
$$

The following result is well-known.

Theorem 6.1. The operator (6.2) is Fredholm if and only if

$$
L(a) \cup M(a) \subset \mathbb{C} \backslash\{0\} .
$$

A proof of the Fredholm property of (6.2) under the condition (6.4) is given in Eskin's book [7]; it is also noted there that (6.1) is necessary. Details of that part of the proof may be found in [16].

Corollary 6.2. Let $a(\tau) \in S_{\mathrm{cl}}^{0}(\mathbb{R})$ be elliptic in the sense $L(a) \subset \mathbb{C} \backslash\{0\}$. Then $(6.2)$ is a Fredholm operator if and only if

$$
0 \notin M(a) .
$$

The union

$$
C(a):=L(a) \cup M(a)
$$

is a continuous and piecewise smooth curve which can be represented as the image under a continuous map $\gamma:[0,1] \rightarrow \mathbb{C}$. If $(6.4)$ holds we have a winding number wind $C(a)$, and there is the well-known relation

$$
\text { ind op }{ }^{+}(a)=\text { wind } C(a) \text {. }
$$

Observe that

$$
a_{0}^{-}=-a_{0}^{+} \Rightarrow 0 \in M(a),
$$

i.e., the operator (6.2) cannot be Fredholm in this case. 
Definition 6.3. A symbol $a(\tau) \in S_{\mathrm{cl}}^{\mu}(\mathbb{R})$ for $\mu \in \mathbb{Z}$ is said to have the antitransmission property if the coefficients $a_{j}^{ \pm}$in the asymptotic expansion (3.8) satisfy the condition

$$
a_{j}^{+}=-a_{j}^{-} \text {for all } j \in \mathbb{N} .
$$

Let $S_{-\operatorname{tr}}^{\mu}(\mathbb{R})$ denote the space of all symbols with the anti-transmission property.

Note that (6.6) is just the opposite of (3.11).

Proposition 6.4. Every $a(\tau) \in S_{\mathrm{cl}}^{\mu}(\mathbb{R})$ can be written in the form

$$
a(\tau)=\frac{1}{2}\left(a_{\mathrm{tr}}(\tau)+a_{-\operatorname{tr}}(\tau)\right)+c(\tau)
$$

for suitable $a_{\mathrm{tr}}(\tau) \in S_{\mathrm{tr}}^{\mu}(\mathbb{R}), a_{-\operatorname{tr}}(\tau) \in S_{-\operatorname{tr}}^{\mu}(\mathbb{R}), c(\tau) \in \mathcal{S}(\mathbb{R})$.

Proof. Similarly as (3.7), (3.8) we form a symbol

$$
b(\tau) \sim \sum_{j=0}^{\infty} \chi(\tau)\left(a_{j}^{-} \theta^{+}(\tau)+a_{j}^{+} \theta^{-}(\tau)\right)(i \tau)^{\mu-j}
$$

belonging to $S_{\mathrm{cl}}^{\mu}(\mathbb{R})$, where $\chi(\tau)$ is some excision function. Then we obviously have $a_{\mathrm{tr}}(\tau):=a(\tau)+b(\tau) \in S_{\mathrm{tr}}^{\mu}(\mathbb{R}), a_{-\operatorname{tr}}(\tau):=a(\tau)-b(\tau) \in S_{-\operatorname{tr}}^{\mu}(\mathbb{R})$, and we obtain the relation $(6.7)$.

Remark 6.5. A symbol $a(\tau) \in S_{\mathrm{cl}}^{\mu}(\mathbb{R})$ has the anti-transmission property exactly when

$$
a_{(\mu-j)}(\tau)=(-1)^{\mu-j+1} a_{(\mu-j)}(-\tau)
$$

for all $\tau \in \mathbb{R} \backslash\{0\}$ and all $j \in \mathbb{N}$.

In fact, the anti-transmission property means that

$$
a_{(\mu-j)}(\tau)=\left(c_{j} \theta^{+}(\tau)-c_{j} \theta^{-}(\tau)\right)(i \tau)^{\mu-j}
$$

for constants $c_{j}:=a_{j}^{+} \in \mathbb{C}$. This yields the relation

$$
\begin{aligned}
a_{(\mu-j)}(-\tau) & =\left(c_{j} \theta^{+}(-\tau)-c_{j} \theta^{-}(-\tau)\right)(-i \tau)^{\mu-j} \\
& =(-1)^{\mu-j}\left(c_{j} \theta^{-}(\tau)-c_{j} \theta^{+}(\tau)\right)=(-1)^{\mu-j+1} a_{(\mu-j)}(\tau),
\end{aligned}
$$

using $\theta^{+}(-\tau)=\theta^{-}(\tau), \theta^{-}(-\tau)=\theta^{+}(\tau)$.

Conversely, from (6.8) we obtain

$$
\begin{aligned}
\left\{a_{j}^{+} \theta^{+}(\tau)+a_{j}^{-} \theta^{-}(\tau)\right\}(i \tau)^{\mu-j} & =(-1)^{\mu-j+1}\left\{a_{j}^{+} \theta^{+}(-\tau)+a_{j}^{-} \theta^{-}(-\tau)\right\}(-i \tau)^{\mu-j} \\
& =\left\{\theta^{-} a_{j}^{+} \theta^{-}(\tau)+a_{j}^{-} \theta^{+}(\tau)\right\}(i \tau)^{\mu-j}
\end{aligned}
$$

This gives us $a_{j}^{+}=-a_{j}^{-}$which are the conditions of Definition 6.3.

Observe that there is also a higher-dimensional analogue of Definition 4.11 for symbols $p(y, t, \eta, \tau) \in S_{\mathrm{cl}}^{\mu}\left(\Omega \times \overline{\mathbb{R}}_{+} \times \mathbb{R}_{y, \tau}\right)$ where instead of (4.18) we ask

$$
D_{y, t}^{\alpha} D_{\eta, \tau}^{\beta}\left\{p_{(\mu-j)}(y, t, \eta, \tau)-(-1)^{\mu-j+1} p_{(\mu-j)}(y, t,-\eta,-\tau)\right\}=0
$$


on $\{(y, t, \eta, \tau): y \in \Omega, t=0, \eta=0, \tau \in \mathbb{R} \backslash\{0\}\}$ for all $\alpha, \beta, j$. This gives us the symbol class $S_{-\operatorname{tr}}^{\mu}\left(\Omega \times \overline{\mathbb{R}}_{+} \times \mathbb{R}^{n}\right)$. There is then a higher-dimensional analogue of Proposition 6.4.

In fact, let $a(y, t, \eta, \tau) \in S_{\mathrm{cl}}^{\mu}\left(\Omega \times \overline{\mathbb{R}}_{+} \times \mathbb{R}^{n}\right)$ be arbitrary, and define the homogeneous components

$$
a_{\mathrm{tr},(\mu-j)}(y, t, \eta, \tau):=a_{(\mu-j)}(y, t, \eta, \tau)+(-1)^{\mu-j} a_{(\mu-j)}(y, t,-\eta,-\tau)
$$

and

$$
a_{-\operatorname{tr},(\mu-j)}(y, t, \eta, \tau):=a_{(\mu-j)}(y, t, \eta, \tau)-(-1)^{\mu-j} a_{(\mu-j)}(y, t,-\eta,-\tau)
$$

for all $j$ and $(y, t, \eta, \tau) \in \Omega \times \overline{\mathbb{R}}_{+} \times \mathbb{R}^{n} \backslash\{0\}$. Then we have

$$
\begin{aligned}
a_{\operatorname{tr},(\mu-j)}(y, t, \eta, \tau)-(-1)^{\mu-j} a_{\operatorname{tr},(\mu-j)}(y, t,-\eta,-\tau) & \\
= & a_{(\mu-j)}(y, t, \eta, \tau)+(-1)^{\mu-j} a_{(\mu-j)}(y, t,-\eta,-\tau) \\
& -(-1)^{\mu-j}\left\{a_{(\mu-j)}(y, t,-\eta-\tau)+(-1)^{\mu-j} a_{(\mu-j)}(y, t, \eta, \tau)\right\}=0,
\end{aligned}
$$

and

$$
\begin{aligned}
a_{-\operatorname{tr},(\mu-j)} & (y, t, \eta, \tau)+(-1)^{\mu-j} a_{-\operatorname{tr},(\mu-j)}(y, t,-\eta,-\tau) \\
= & a_{(\mu-j)}(y, t, \eta, \tau)-(-1)^{\mu-j} a_{(\mu-j)}(y, t,-\eta,-\tau) \\
& +(-1)^{\mu-j}\left\{a_{(\mu-j)}(y, t,-\eta,-\tau)-(-1)^{\mu-j} a_{(\mu-j)}(y, t, \eta, \tau)\right\}=0
\end{aligned}
$$

for all $j$ and $(y, t, \eta, \tau) \in \Omega \times \overline{\mathbb{R}}_{+} \times\left(\mathbb{R}^{n} \backslash\{0\}\right)$. In other words, if we define

$$
\begin{aligned}
a_{\operatorname{tr}}(y, t, \eta, \tau) & \sim \sum_{j=0}^{\infty} \chi(\eta, \tau) a_{\operatorname{tr},(\mu-j)}(y, t, \eta, \tau), \\
a_{-\operatorname{tr}}(y, t, \eta, \tau) & \sim \sum_{j=0}^{\infty} \chi(\eta, \tau) a_{-\operatorname{tr},(\mu-j)}(y, t, \eta, \tau),
\end{aligned}
$$

then $a_{\mathrm{tr}}$ has the transmission property, $a_{-\operatorname{tr}}$ the anti-transmission property; here $\chi(\eta, \tau)$ is any excision function. Thus we have proved the following result.

Proposition 6.6. Every symbol $a(y, t, \eta, \tau) \in S_{\mathrm{cl}}^{\mu}\left(\Omega \times \overline{\mathbb{R}}_{+} \times \mathbb{R}^{n}\right)$ can be written in the form

$$
a(y, t, \eta, \tau)=\frac{1}{2}\left\{a_{\operatorname{tr}}(y, t, \eta, \tau)+a_{-\operatorname{tr}}(y, t, \eta, \tau)\right\}+c(y, t, \eta, \tau)
$$

for symbols $a_{\operatorname{tr}}(y, t, \eta, \tau) \in S_{\operatorname{tr}}^{\mu}\left(\Omega \times \overline{\mathbb{R}}_{+} \times \mathbb{R}^{n}\right), a_{-\operatorname{tr}}(y, t, \eta, \tau) \in S_{-\operatorname{tr}}^{\mu}\left(\Omega \times \overline{\mathbb{R}}_{+} \times \mathbb{R}^{n}\right)$ (uniquely determined $\left.\bmod S^{-\infty}\left(\Omega \times \overline{\mathbb{R}}_{+} \times \mathbb{R}^{n}\right)\right), c(y, t, \eta, \tau) \in S^{-\infty}\left(\Omega \times \overline{\mathbb{R}}_{+} \times \mathbb{R}^{n}\right)$.

The role of those considerations here is not to really carry out a calculus of BVPs having the anti-transmission property. As noted at the beginning such a calculus is possible indeed, however, based on tools from the cone and edge calculus that go beyond the scope of this exposition. Let us only mention that for such a program we need to reorganise both the symbolic structure and the 
operator conventions of our operators as well as the spaces that substitute the standard Sobolev spaces. Details on the new boundary symbolic calculus for zero order operators on the half-axis may be found in [7], and in [20]. Concerning the cone and edge calculus in general, cf. [17], [18], [19].

\section{References}

[1] S. Agmon, A. Douglis, L. Nirenberg,Estimates near the boundary for solutions of elliptic partial differential equations satisfying general boundary conditions I, Comm. Pure Appl. Math., 12, (1959), 623-727.

[2] M.F.Atiyah, R. Bott, The index problem for manifolds with boundary, in: Coll. Differential Analysis, Tata Institute Bombay, Oxford University Press, (1964), 175-186.

[3] M.F. Atiyah, V. Patodi, I.M. Singer, Spectral asymmetry and Riemannian geometry I, II, III, Math. Proc. Cambridge Philos. Soc., 77,78,79, (1975, 1976, 1976), 43-69, 405-432, 315-330.

[4] L. Boutet de Monvel, Boundary problems for pseudo-differential operators, Acta Math., 126, (1971), 11-51.

[5] L. Boutet de Monvel, Comportement d'un opérateur pseudo-différentiel sur une variété à bord, J. Anal. Math., 17, (1966), 241-304.

[6] Ju.V Egorov, B.-W. Schulze, Pseudo-differential operators, singularities, applications, Operator Theory, Advances and Applications, 93, Birkhäuser, Basel, 1997.

[7] G.I. Eskin, Boundary value problems for elliptic pseudodifferential equations, Math. Monographs, 52, Amer. Math. Soc. (1980).

[8] G. Grubb, Functional calculus of pseudo-differential boundary problems, Birkhäuser, Boston, 1996. 69.

[9] G. Grubb, Pseudo-differential boundary value problems in $L_{p}$, Comm. Partial Differential Equations, 15, (1990), 289-340.

[10] G. Harutjunjan, B.-W. Schulze, Elliptic Mixed, Transmission and Singular Crack Problems, European Mathematical Soc.,Zürich, 2008.

[11] K. Jänich, Vektorraumbündel und der Raum der Fredholm-Operatoren, Math. Ann., 161, (1965), 129-142.

[12] D. Kapanadze, B.-W. Schulze, Crack theory and edge singularities, Kluwer Academic Publ.,Dordrecht , 2003.

[13] J.-L.Lions, E. Magenes, Problèmes aux limites non homogènes et applications 1 , Dunod, Paris, 1968.

[14] P.A. Myshkis, On an algebra generated by two-sided pseudodifferential operators on a manifold, Uspechi Mat. Nauk., 31, 4, (1976), 269-270.

[15] S. Rempel, B.-W. Schulze, Index theory of elliptic boundary problems, AkademieVerlag, Berlin, 1982.

[16] S. Rempel, B.-W. Schulze, Parametrices and boundary symbolic calculus for elliptic boundary problems without transmission property, Math. Nachr., 105, (1982), 45-149.

[17] B.-W. Schulze, Pseudo-differential operators on manifolds with singularities, NorthHolland, Amsterdam, 1991 
[18] B.-W. Schulze, Boundary value problems and singular pseudo-differential operators, J. Wiley, Chichester, 1998.

[19] B.-W. Schulze, Operator algebras with symbol hierarchies on manifolds with singularities, Advances in Partial Differential Equations (Approaches to Singular Analysis) (J. Gil, D. Grieser, and Lesch M., eds.), Oper. Theory Adv. Appl., Birkhäuser Verlag, Basel, 2001, pp. 167-207.

[20] B.-W. Schulze, Pseudo-differential boundary value problems, conical singularities, and asymptotics, Akademie Verlag, Berlin, 1994

[21] B.-W. Schulze, An algebra of boundary value problems not requiring ShapiroLopatinskij conditions, J. Funct. Anal., 179, (2001), 374-408.

[22] B.-W. Schulze, J. Seiler, The edge algebra structure of boundary value problems, Annals of Global Analysis and Geometry, 22, (2002), 197-265.

[23] B.-W. Schulze, J. Seiler, Pseudodifferential boundary value problems with global projection conditions, J. Funct. Anal., 206, 2, (2004), 449-498.

[24] B.-W. Schulze, Topologies and invertibility in operator spaces with symbolic structures, Teubner Texte zur Mathematik, 111, "Problems and Methods in Mathematical Physics" (1989), 257-270.

[25] B.-W. Schulze, , Pseudo-differential operators on manifolds with edges, TeubnerTexte zur Mathematik, 112, Symp. "Partial Differential Equations", Holzhau 1988, (1989), 259-287.

[26] B.-W. Schulze, The Mellin pseudo-differential calculus on manifolds with corners, Teubner-Texte zur Mathematik, 131, Symp. "Analysis in Domains and on Manifolds with Singularities", Breitenbrunn 1990, (1990), 208-289.

[27] B.-W. Schulze and J. Seiler, Edge operators with conditions of Toeplitz type, J. of the Inst. Math. Jussieu, 5, 1, (2006), 101-123.

[28] J. Seiler, Continuity of edge and corner pseudo-differential operators, Math. Nachr., 205, (1999), 163-182.

[29] M.I. Vishik and G.I. Eskin, Convolution equations in bounded domains in spaces with weighted norms, Mat. Sb., 69, 1, (1966), 65-110.

Universität Potsdam, Institut für Mathematik, Am Neuen Palais 10, 14469 Potsdam, Germany

E-mail address: schulze@math.uni-potsdam.de 